\title{
BSU-8242 As-Run Thermal Analysis
}

\section{July 2021}

Cody Hale

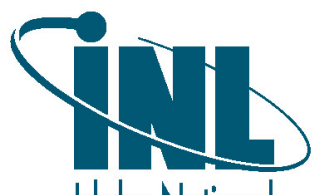

Idaho National

Laboratory 


\section{DISCLAIMER}

This information was prepared as an account of work sponsored by an agency of the U.S. Government. Neither the U.S. Government nor any agency thereof, nor any of their employees, makes any warranty, expressed or implied, or assumes any legal liability or responsibility for the accuracy, completeness, or usefulness, of any information, apparatus, product, or process disclosed, or represents that its use would not infringe privately owned rights. References herein to any specific commercial product, process, or service by trade name, trade mark, manufacturer, or otherwise, does not necessarily constitute or imply its endorsement, recommendation, or favoring by the U.S. Government or any agency thereof. The views and opinions of authors expressed herein do not necessarily state or reflect those of the U.S. Government or any agency thereof. 


\title{
BSU-8242 As-Run Thermal Analysis
}

\author{
Cody Hale
}

July 2021

\section{Idaho National Laboratory Idaho Falls, Idaho 83415}

\author{
http://www.inl.gov
}

\author{
Prepared for the \\ U.S. Department of Energy \\ Under DOE Idaho Operations Office \\ Contract DE-AC07-05ID14517
}




\begin{tabular}{|c|c|}
\hline 1. Effective Date & $07 / 22 / 2021$ \\
\hline $\begin{array}{l}\text { 2. Does this ECAR involve a } \\
\text { Safety SSC? }\end{array}$ & NO \\
\hline $\begin{array}{l}\text { 3. Safety SSC Determination } \\
\text { Document ID }\end{array}$ & $\mathrm{N} / \mathrm{A}$ \\
\hline 4. SSC ID & $\mathrm{N} / \mathrm{A}$ \\
\hline 5. Project No. & 32349 \\
\hline 6. Engineering Job (EJ) No. & $\mathrm{N} / \mathrm{A}$ \\
\hline 7. Building & TRA-670 \\
\hline 8. Site Area & ATR Complex \\
\hline
\end{tabular}

Professional Engineer's Stamp

N/A

per LWP-10010, Sec 4.1, par. cc

\section{Objective / Purpose}

This Engineering Calculations and Analysis Report (ECAR) documents the calculated as-run thermal analysis using the calculated as-run heat rates. The heat rates were calculated using as-run reactor data for the average lobe powers over the given operating cycle. The heat rates were then input into the thermal models that were generated during the design phase for the experiment (See ECAR-3575 [1]). No as-built data was provided and therefore not incorporated into the models. Nominal gas gaps and dimensions were used. For gas gap sensitivities, refer to ECAR-3575 [1]. 10. If revision, please state the reason and list sections and/or page being affected.

\section{$\mathrm{N} / \mathrm{A}$}

\section{Conclusion / Recommendations}

As-run heating rates calculated in ECAR-4341 [2] and ECAR-4951 [3] were input into the models created during the design of the experiment to evaluate the as-run temperatures. The models used are described in ECAR-3575 [1]. The results are shown in Section 8.2. Melt wires were used in the experiment and a comparison has been made to the calculated design and calculated as-run temperatures which can be found in Section 8.3. 


\section{CONTENTS}

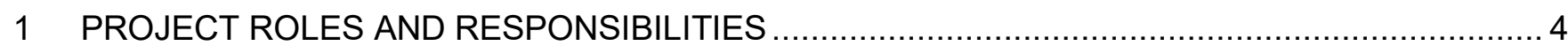

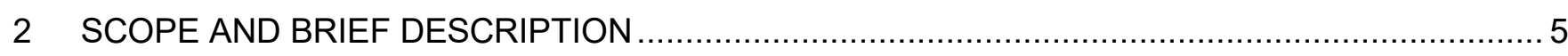

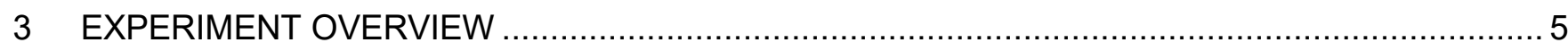

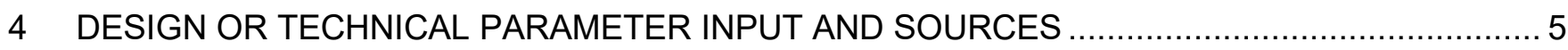

5 RESULTS OF LITERATURE SEARCHES AND OTHER BACKGROUND DATA .....................6

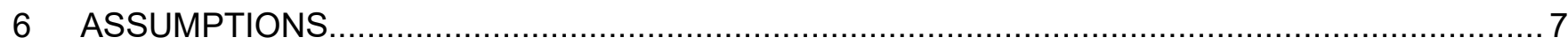

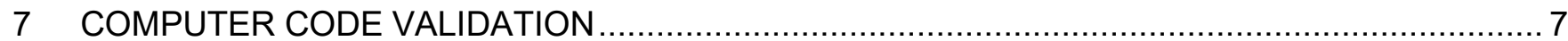

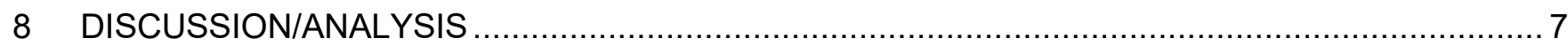

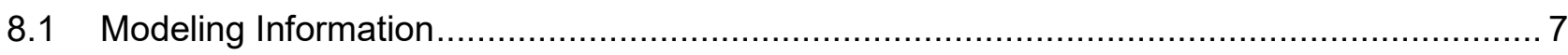

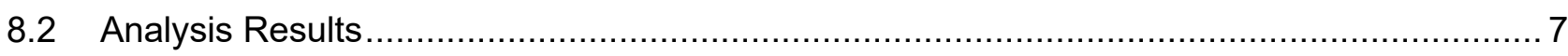

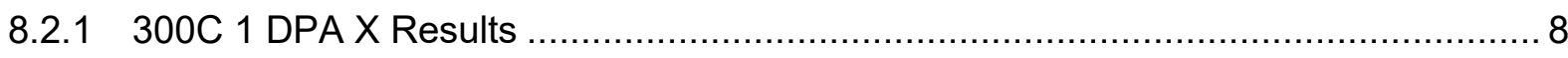

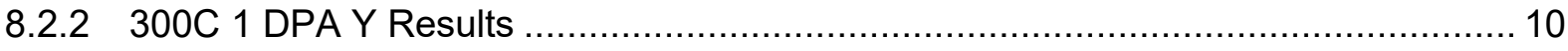

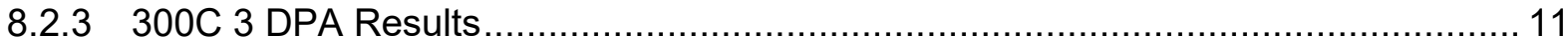

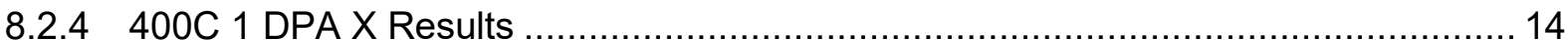

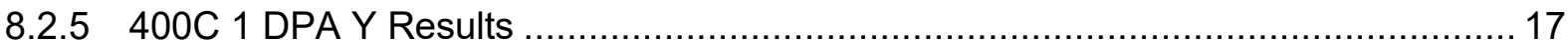

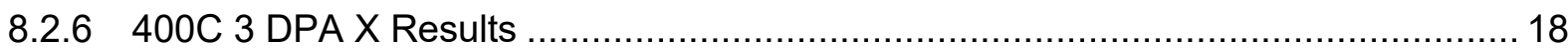

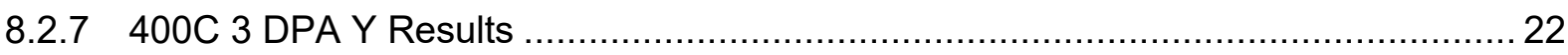

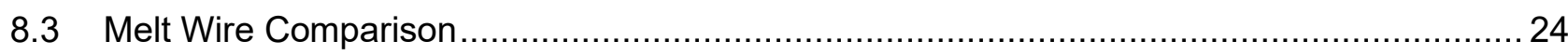

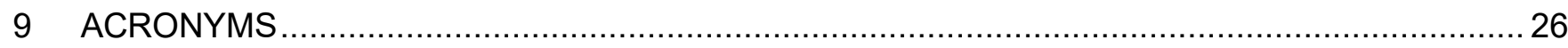

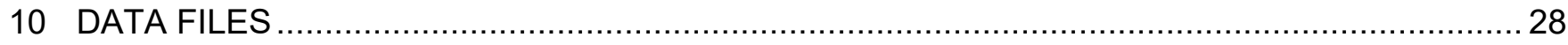

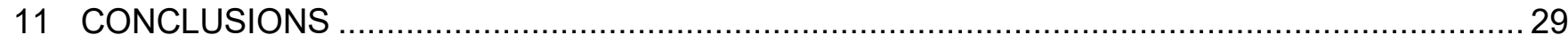

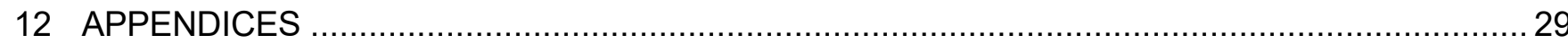

\section{TABLES}

Table 5-1. Drawing List

Table 8-1: Specimen Temperatures for Capsule 300C 1 DPA X in Cycle 164A ................................ 8

Table 8-2: Specimen Temperatures for Capsule 300C 1 DPA Y in Cycle 164A ........................... 10

Table 8-3: Specimen Temperatures for Capsule 300C 3 DPA in Cycles 164B, 166A, and 166B ........ 13

Table 8-4: Specimen Temperatures for Capsule 400C 1 DPA X in Cycle 164A .............................. 15

Table 8-5: Specimen Temperatures for Capsule 400C 1 DPA Y in Cycle 164A ............................ 17

Table 8-6: Specimen Temperatures for Capsule 400C 3 DPA X in Cycles 164B, 166A, and 166B ...... 20

Table 8-7: Specimen Temperatures for Capsule 400C 3 DPA Y in Cycle 164B, 166A, and 166B ........24

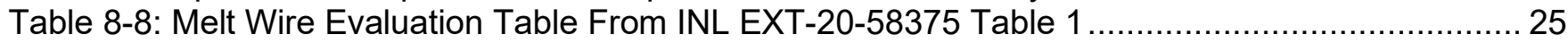

Table 8-9: Melt Wire Temperature Based on Specimen Temperatures Calculated in ECAR-3575...... 25

Table 8-10: Summary of Average Calculated As-Run Temperatures . 


\section{FIGURES}

Figure 8-1: Specimen Temperatures for Capsule 300C 1 DPA X in Cycle 164A ............................. 8

Figure 8-2: Specimen Temperatures for Capsule 300C 1 DPA Y in Cycle 164A ........................... 10

Figure 8-3: Specimen Temperatures for Capsule 300C 3 DPA in Cycle 164B .............................. 11

Figure 8-4: Specimen Temperatures for Capsule 300C 3 DPA in Cycle 166A ................................ 12

Figure 8-5: Specimen Temperatures for Capsule 300C 3 DPA in Cycle 166B ............................... 12

Figure 8-6: Specimen Temperatures for Capsule 400C 1 DPA $X$ in Cycle 164A ............................. 14

Figure 8-7: Specimen Temperatures for Capsule 400C 1 DPA Y in Cycle 164A ............................. 17

Figure 8-8: Specimen Temperatures for Capsule 400C 3 DPA X in Cycle 164B ............................. 18

Figure 8-9: Specimen Temperatures for Capsule 400C 3 DPA X in Cycle 166A ........................... 19

Figure 8-10: Specimen Temperatures for Capsule 400C 3 DPA X in Cycle 166B ............................19

Figure 8-11: Specimen Temperatures for Capsule 400C 3 DPA Y in Cycle 164B ...........................22

Figure 8-12: Specimen Temperatures for Capsule 400C 3 DPA Y in Cycle 166A ............................23

Figure 8-13: Specimen Temperatures for Capsule 400C 3 DPA Y in Cycle 166B ........................... 23 


\section{PROJECT ROLES AND RESPONSIBILITIES}

\begin{tabular}{|l|l|l|l|}
\hline \multicolumn{1}{|c|}{ Project Role } & \multicolumn{1}{c|}{ Name (Typed) } & Organization & Pages covered (if applicable) \\
\hline Performer & Cody Hale & C140 & All \\
\hline Checker $^{\mathrm{a}}$ & Dan Chapman & C130 & All \\
\hline Independent Reviewer & N/A & N/A & N/A \\
\hline CUI Reviewer & Katie Anderson & C640 & All \\
\hline Manager $^{d}$ & Richard Howard & C140 & All \\
\hline Requestor $^{\mathrm{e}}$ & Donna Guillen & B611 & All \\
\hline Nuclear Safety & N/A & N/A & N/A \\
\hline Document Owner & Donna Guillen & B611 & All \\
\hline
\end{tabular}

\section{Responsibilities:}

a. Confirmation of completeness, mathematical accuracy, and correctness of data and appropriateness of assumptions.

b. Concurrence of method or approach. See definition, LWP-10106.

c. Concurrence with the document's markings in accordance with LWP-11202.

d. Concurrence of procedure compliance. Concurrence with method/approach and conclusion.

e. Concurrence with the document's assumptions and input information. See definition of Acceptance, LWP-10200. 


\section{SCOPE AND BRIEF DESCRIPTION}

This Engineering Calculations and Analysis Report (ECAR) documents the calculated as-run thermal analysis using the calculated as-run heat rates. The heat rates were calculated using as-run reactor data for the average lobe powers over the given operating cycle. The heat rates were then input into the thermal models that were generated during the design phase for the experiment (See ECAR-3575 [1]). No as-built data was provided and therefore not incorporated into the models. Nominal gas gaps and dimensions were used. For gas gap sensitivities, refer to ECAR-3575 [1].

\section{EXPERIMENT OVERVIEW}

The objective of this experiment is to assess the viability of using alloys manufactured by powder metallurgy and hot isostatic pressing (PM-HIP) as nuclear reactor internals, to enhance the weldability and inspectability of these components. The superior properties of PM-HIP alloys make them potential candidates for structural materials in nuclear reactor technologies, including light water reactors (LWRs), advanced light water reactors (ALWRs), small modular reactors (SMRs), and advanced (e.g. Generation IV) reactor designs. However, little is known about the irradiation response of PM-HIP alloys, particularly in comparison to that of conventionally manufactured alloys. The purpose of this experiment is to test and understand the irradiation effect on PM-HIP alloys.

To test these alloys, the experiment sponsor has provided INL with three different types of specimens. The first type of specimen is a round "dog bone" shaped tensile specimen with an overall length of 3 in. and a gauge diameter of 0.25 in. The second specimen type is a Compact Tensile (CT) specimen which is cube shaped with the dimension of $0.394 \mathrm{in}$. X $0.378 \mathrm{in.} \times 0.157 \mathrm{in}$. The third type of specimen is a round Transmission Electron Microscope (TEM) disk with a diameter of 0.118 in. All the TEM's have a thickness of 0.006 in. except for the hafnium-aluminum materials which have a thickness of 0.012 in (twice as thick as the other TEM materials).

In order to house the desired quantity of materials, some of the specimens had to be distributed between two capsules with the same desired irradiation condition. The specimens that have been split into two capsules are the $300^{\circ} \mathrm{C} 1 \mathrm{dpa}$ specimens, $400^{\circ} \mathrm{C} 1 \mathrm{dpa}$ specimens, and the $400^{\circ} \mathrm{C} 3 \mathrm{dpa}$ specimens. This brings the total number of capsules to seven. However, the $300^{\circ} \mathrm{C}$ and $400^{\circ} \mathrm{C} 1 \mathrm{dpa}$ capsules are combined into two experiment assemblies for a total of five assemblies. To distinguish between the two capsules of the same type, an " $X$ " or " $Y$ " designator has been assigned to them. For the remainder of this ECAR the seven capsules will be referred to as $300 \mathrm{C} 1 \mathrm{dpa} X, 300 \mathrm{C} 1 \mathrm{dpa} Y$, 400C $1 \mathrm{dpa} X, 400 \mathrm{C} 1 \mathrm{dpa} Y, 400 \mathrm{C} 3 \mathrm{dpa} X, 400 \mathrm{C} 3 \mathrm{dpa} Y$, and 300C $3 \mathrm{dpa}$. The five experiment

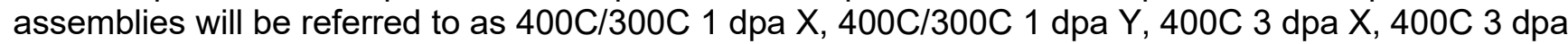
$\mathrm{Y}$, and 300C 3 dpa.

\section{DESIGN OR TECHNICAL PARAMETER INPUT AND SOURCES}

The models used in this analysis were developed during the design phase of this experiment. Information regarding those models can be found in ECAR-3575 [1]. Two different ECAR's were created to document the heating rates; one for the 1 dpa capsules which is ECAR-4341 [2], and one for the 3 dpa capsules which is ECAR-4951 [3]. 


\section{RESULTS OF LITERATURE SEARCHES AND OTHER BACKGROUND DATA}

The drawings used in these analyses are referenced below in Table 1.

Table 5-1. Drawing List

\begin{tabular}{|c|c|c|}
\hline $\begin{array}{l}\text { Drawing } \\
\text { Number }\end{array}$ & Rev. & Description \\
\hline 602380 & 1 & UTAH STATE UNIVERSITY SPECIMEN DETAILS \\
\hline 605765 & 2 & $\begin{array}{l}\text { ATR NSUF BOISE EXPERIMENT } 8242 \text { INBOARD A CAPSULE } \\
\text { COMPONENT ASSEMBLIES AND DETAILS }\end{array}$ \\
\hline 605766 & 0 & $\begin{array}{l}\text { ATR NSUF BOISE EXPERIMENT } 8242 \text { 300C-1 DPA X INBOARD A } \\
\text { CAPSULE CT AND TEM SPECIMEN ASSEMBLY }\end{array}$ \\
\hline 605767 & 1 & $\begin{array}{l}\text { ATR NSUF BOISE EXPERIMENT } 8242 \text { 300C-1 DPA Y INBOARD A } \\
\text { CAPSULE CT AND TEM SPECIMEN ASSEMBLY }\end{array}$ \\
\hline 605768 & 1 & $\begin{array}{l}\text { ATR NSUF BOISE EXPERIMENT } 8242 \text { 300C-3 DPA INBOARD A } \\
\text { CAPSULE CT AND TEM SPECIMEN ASSEMBLY }\end{array}$ \\
\hline 605769 & 1 & $\begin{array}{l}\text { ATR NSUF BOISE EXPERIMENT } 8242 \text { 400C-1 DPA X INBOARD A } \\
\text { CAPSULE CT AND TEM SPECIMEN ASSEMBLY }\end{array}$ \\
\hline 605770 & 0 & $\begin{array}{l}\text { ATR NSUF BOISE EXPERIMENT } 8242 \text { 400C-1 DPA Y INBOARD A } \\
\text { CAPSULE CT AND TEM SPECIMEN ASSEMBLY }\end{array}$ \\
\hline 605771 & 2 & $\begin{array}{l}\text { ATR NSUF BOISE EXPERIMENT } 8242 \text { 400C-3 DPA X INBOARD A } \\
\text { CAPSULE CT AND TEM SPECIMEN ASSEMBLY }\end{array}$ \\
\hline 605772 & 1 & $\begin{array}{l}\text { ATR NSUF BOISE EXPERIMENT } 8242 \text { 400C-3 DPA Y INBOARD A } \\
\text { CAPSULE CT AND TEM SPECIMEN ASSEMBLY }\end{array}$ \\
\hline 605773 & 1 & ATR BSU-8242 Experiment Inboard A Capsule Drawing Tree \\
\hline 605774 & 2 & $\begin{array}{l}\text { ATR NSUF BOISE EXPERIMENT } 8242 \text { SPECIMEN ASSEMBLY AND } \\
\text { DETAILS }\end{array}$ \\
\hline 605775 & 0 & $\begin{array}{l}\text { ATR NSUF BOISE EXPERIMENT } 8242 \text { INBOARD A CAPSULE } \\
\text { TENSILE SPECIMEN ASSEMBLIES }\end{array}$ \\
\hline 605776 & 0 & $\begin{array}{l}\text { ATR NSUF BOISE EXPERIMENT } 8242 \text { 300C-1 DPA X INBOARD A } \\
\text { CAPSULE ASSEMBLY }\end{array}$ \\
\hline 605777 & 0 & $\begin{array}{l}\text { ATR NSUF BOISE EXPERIMENT } 8242 \text { 300C-3 DPA INBOARD A } \\
\text { CAPSULE ASSEMBLY }\end{array}$ \\
\hline 605778 & 0 & $\begin{array}{l}\text { ATR NSUF BOISE EXPERIMENT } 8242 \text { 300C-1 DPA Y INBOARD A } \\
\text { CAPSULE ASSEMBLY }\end{array}$ \\
\hline 605779 & 1 & $\begin{array}{l}\text { ATR NSUF BOISE EXPERIMENT } 8242 \text { 400C-1 DPA X INBOARD A } \\
\text { CAPSULE ASSEMBLY }\end{array}$ \\
\hline 605780 & 0 & $\begin{array}{l}\text { ATR NSUF BOISE EXPERIMENT } 8242 \text { 400C-1 DPA Y INBOARD A } \\
\text { CAPSULE ASSEMBLY }\end{array}$ \\
\hline 605781 & 0 & $\begin{array}{l}\text { ATR NSUF BOISE EXPERIMENT } 8242 \text { 400C-3 DPA Y INBOARD A } \\
\text { CAPSULE ASSEMBLY }\end{array}$ \\
\hline 605782 & 1 & $\begin{array}{l}\text { ATR NSUF BOISE EXPERIMENT } 8242 \text { 400C-3 DPA X INBOARD A } \\
\text { CAPSULE ASSEMBLY }\end{array}$ \\
\hline 605824 & 1 & $\begin{array}{l}\text { ATR NSUF BOISE EXPERIMENT } 8242 \text { INBOARD A CAPSULE 300C- } \\
1 \text { DPA X AND 400C-1 DPA X TEST TRAIN ASSEMBLY }\end{array}$ \\
\hline 605 & 1 & $\begin{array}{l}\text { ATR NSUF BOISE EXPERIMENT } 8242 \text { INBOARD A CAPSULE 300C- } \\
1 \text { DPA Y AND 400C-1 DPA Y TEST TRAIN ASSEMBLY }\end{array}$ \\
\hline
\end{tabular}


BSU-8242 As-Run Thermal Analysis

\begin{tabular}{|c|c|l|}
\hline $\begin{array}{l}\text { Drawing } \\
\text { Number }\end{array}$ & Rev. & \multicolumn{1}{|c|}{ Description } \\
\hline 605826 & 2 & $\begin{array}{l}\text { ATR NSUF BOISE EXPERIMENT 8242 INBOARD A CAPSULE 300C- } \\
\text { 3 DPA TEST TRAIN ASSEMBLY }\end{array}$ \\
\hline 605827 & 2 & $\begin{array}{l}\text { ATR NSUF BOISE EXPERIMENT 8242 INBOARD A CAPSULE 400C- } \\
\text { 3 DPA X TEST TRAIN ASSEMBLY }\end{array}$ \\
\hline 605828 & 1 & $\begin{array}{l}\text { ATR NSUF BOISE EXPERIMENT 8242 INBOARD A CAPSULE 400C- } \\
\text { 3 DPA Y TEST TRAIN ASSEMBLY }\end{array}$ \\
\hline 605829 & 0 & $\begin{array}{l}\text { ATR NSUF BOISE EXPERIMENT 8242 INBOARD A CAPSULE TEST } \\
\text { TRAIN INSTALLATION }\end{array}$ \\
\hline
\end{tabular}

\section{ASSUMPTIONS}

1. All dimensions are based on nominal drawing values.

2. Heat rates input into the models are the calculated average heat rates for the given cycle.

\section{COMPUTER CODE VALIDATION}

A finite element heat transfer analysis of the BSU-8242 experiment was performed using ABAQUS version 6.14-2 on a SGI ICE X distributed memory cluster with 972 compute nodes ("falcon" on the INL network). The operating system is SUSE Linux Enterprise Server 12 Service Pack 4, and each compute node has two 18-core $2.10 \mathrm{GHz}$ Intel Xeon processors. ABAQUS has been validated for thermal analysis by solving several test problems and verifying the results against analytical solutions provided in heat transfer textbooks. A complete description of the validation test problems is given in ECAR-131. Scripts were developed to automate the execution, data collection, and relative error calculation for each test problem. The scripts were run on computer "falcon" and a report file containing the results of validation testing was automatically generated (Appendix $\mathrm{C}$ ). The test results meet the acceptance criterion that the relative error is less than $3 \%$. Formal validation of Mathcad applications is not required, instead random hand calculations are performed during checking to verify that the computer-generated output is correct, as discussed in Appendix E in LWP-10200.

\section{DISCUSSIONIANALYSIS}

\subsection{Modeling Information}

For modeling information refer to ECAR-3575 [1]. The only difference is the heating rates input into the models which are shown in Appendix B.

\subsection{Analysis Results}

The following results are provided for each capsule in its respective section. The results are provided graphically to give an overall view of the specimen temperatures and how they compare to the target temperature. However, some of the capsules have a large number of specimens making it difficult to see what the temperatures are for a given specimen; therefore, they have also been provided in a table form. The maximum temperature refers to the maximum nodal temperature using the cycle-average 
heat rate. The average temperature refers to the volume weighted average temperature using the cycle-average heat rate. The maximum and average temperature for the tensile bars is taken in the gauge section only.

\subsubsection{C 1 DPA X Results}

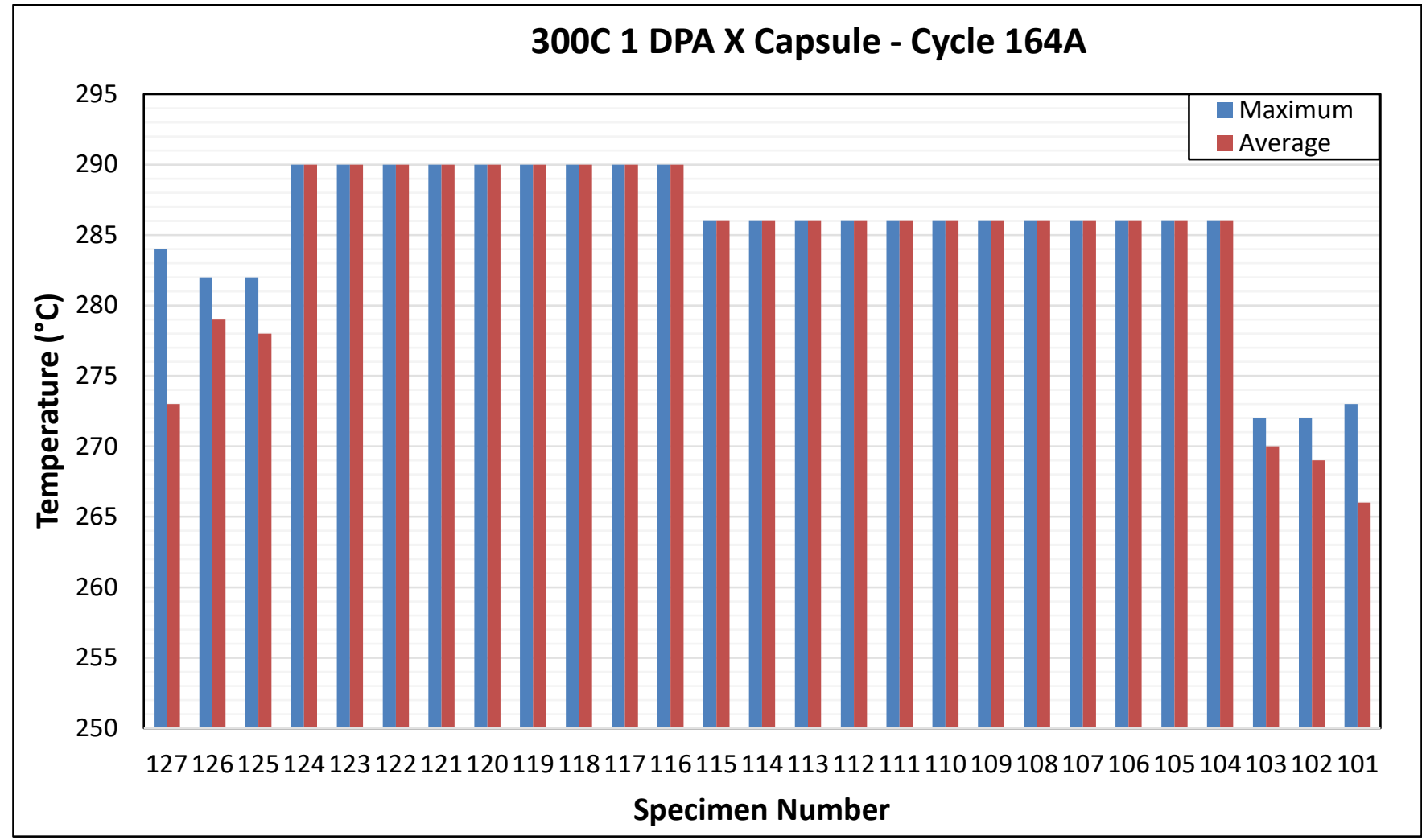

Figure 8-1: Specimen Temperatures for Capsule 300C 1 DPA X in Cycle 164A

Table 8-1: Specimen Temperatures for Capsule 300C 1 DPA X in Cycle 164A

\begin{tabular}{|c|c|c|c|}
\hline \multirow{2}{*}{ Specimen/Material } & $\begin{array}{c}\text { Specimen } \\
\text { Number }\end{array}$ & \multicolumn{2}{|c|}{$\begin{array}{c}\text { Cycle 164A } \\
\text { Temperature } \\
{\left[{ }^{\circ} \mathbf{C}\right]}\end{array}$} \\
\cline { 3 - 4 } & 127 & 284 & 273 \\
\hline RT-SA508 PM-HIP & 126 & 282 & 279 \\
\hline CT-SA508 Forged & 125 & 282 & 278 \\
\hline CT-SA508 PM-HIP & 124 & 290 & 290 \\
\hline $\begin{array}{c}\text { TEM-USU-300-1- } \\
20\end{array}$ & 123 & 290 & 290 \\
\hline $\begin{array}{c}\text { TEM-USU-300-1- } \\
20\end{array}$ & 122 & 290 & 290 \\
\hline $\begin{array}{c}\text { TEM-USU-300-1- } \\
20\end{array}$ & 121 & 290 & 290 \\
\hline $\begin{array}{c}\text { TEM-USU-300-1- } \\
28\end{array}$ & & &
\end{tabular}




\begin{tabular}{|cc|}
\hline $\begin{array}{c}\text { TEM-10200-1, Rev. } 11 \\
11 / 20 / 2019\end{array}$ & ENGINEERING CALCULATIONS AND ANALYSIS Rev. 0 \\
BSU-8242 As-Run Thermal Analysis 9 of 29 \\
\hline
\end{tabular}

\begin{tabular}{|c|c|c|c|}
\hline \multirow[t]{2}{*}{ Specimen/Material } & \multirow[t]{2}{*}{$\begin{array}{c}\text { Specimen } \\
\text { Number }\end{array}$} & \multicolumn{2}{|c|}{$\begin{array}{c}\text { Cycle 164A } \\
\text { Temperature } \\
{\left[{ }^{\circ} \mathrm{C}\right]}\end{array}$} \\
\hline & & Max & Avg \\
\hline $\begin{array}{c}\text { TEM-USU-300-1- } \\
28\end{array}$ & 120 & 290 & 290 \\
\hline $\begin{array}{c}\text { TEM-USU-300-1- } \\
28\end{array}$ & 119 & 290 & 290 \\
\hline $\begin{array}{l}\text { TEM-USU-300-1- } \\
36\end{array}$ & 118 & 290 & 290 \\
\hline $\begin{array}{l}\text { TEM-USU-300-1- } \\
36\end{array}$ & 117 & 290 & 290 \\
\hline $\begin{array}{l}\text { TEM-USU-300-1- } \\
36\end{array}$ & 116 & 290 & 290 \\
\hline $\begin{array}{c}\text { TEM-SA508 } \\
\text { Forged }\end{array}$ & 115 & 286 & 286 \\
\hline $\begin{array}{l}\text { TEM-SA508 } \\
\text { Forged }\end{array}$ & 114 & 286 & 286 \\
\hline $\begin{array}{l}\text { TEM-SA508 } \\
\text { Forged }\end{array}$ & 113 & 286 & 286 \\
\hline $\begin{array}{l}\text { TEM-SA508 } \\
\text { Forged }\end{array}$ & 112 & 286 & 286 \\
\hline $\begin{array}{l}\text { TEM-SA508 } \\
\text { Forged }\end{array}$ & 111 & 286 & 286 \\
\hline $\begin{array}{l}\text { TEM-SA508 } \\
\text { Forged }\end{array}$ & 110 & 286 & 286 \\
\hline $\begin{array}{c}\text { TEM-SA508 PM- } \\
\text { HIP }\end{array}$ & 109 & 286 & 286 \\
\hline $\begin{array}{c}\text { TEM-SA508 PM- } \\
\text { HIP }\end{array}$ & 108 & 286 & 286 \\
\hline $\begin{array}{c}\text { TEM-SA508 PM- } \\
\text { HIP }\end{array}$ & 107 & 286 & 286 \\
\hline $\begin{array}{c}\text { TEM-SA508 PM- } \\
\text { HIP }\end{array}$ & 106 & 286 & 286 \\
\hline $\begin{array}{c}\text { TEM-SA508 PM- } \\
\text { HIP }\end{array}$ & 105 & 286 & 286 \\
\hline $\begin{array}{c}\text { TEM-SA508 PM- } \\
\text { HIP }\end{array}$ & 104 & 286 & 286 \\
\hline CT-SA508 PM-HIP & 103 & 272 & 270 \\
\hline CT-SA508 PM-HIP & 102 & 272 & 269 \\
\hline RT-SA508 PM-HIP & 101 & 273 & 266 \\
\hline
\end{tabular}




\subsubsection{C 1 DPA Y Results}

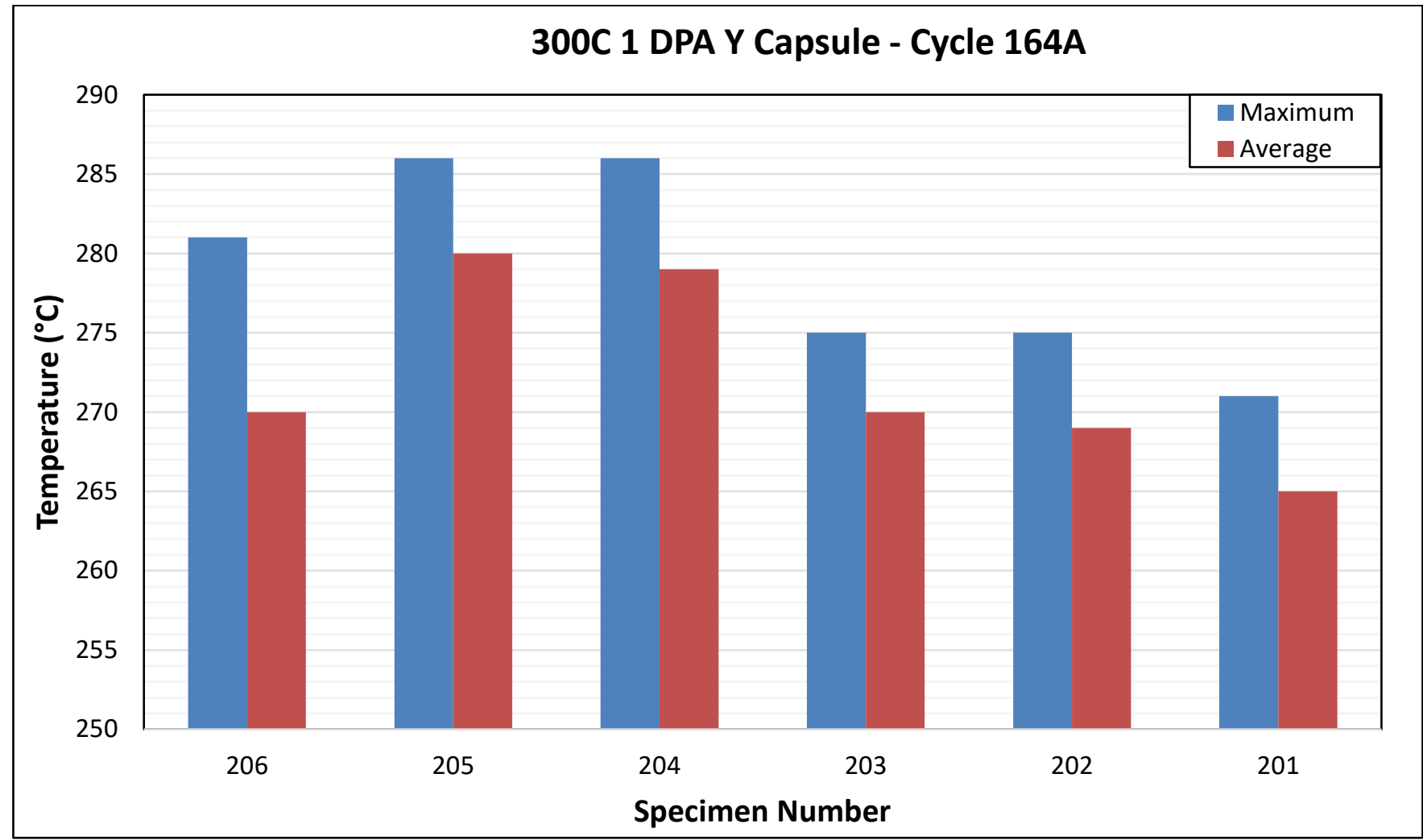

Figure 8-2: Specimen Temperatures for Capsule 300C 1 DPA Y in Cycle 164A

Table 8-2: Specimen Temperatures for Capsule 300C 1 DPA Y in Cycle 164A

\begin{tabular}{|c|c|c|c|}
\hline \multirow{2}{*}{ Specimen/Material } & $\begin{array}{c}\text { Specimen } \\
\text { Number }\end{array}$ & \multicolumn{2}{|c|}{$\begin{array}{c}\text { Cycle 164A } \\
\text { Temperature } \\
{\left[{ }^{\circ} \mathrm{C}\right]}\end{array}$} \\
\cline { 3 - 4 } & & Max & Avg \\
\hline RT-SA508 Forged & 206 & 281 & 270 \\
\hline CT-316L Cast & 205 & 286 & 280 \\
\hline CT-316L Cast & 204 & 286 & 279 \\
\hline CT-316L PM-HIP & 203 & 275 & 270 \\
\hline CT-316L PM-HIP & 202 & 275 & 269 \\
\hline RT-SA508 Forged & 201 & 271 & 265 \\
\hline
\end{tabular}




\subsubsection{C 3 DPA Results}

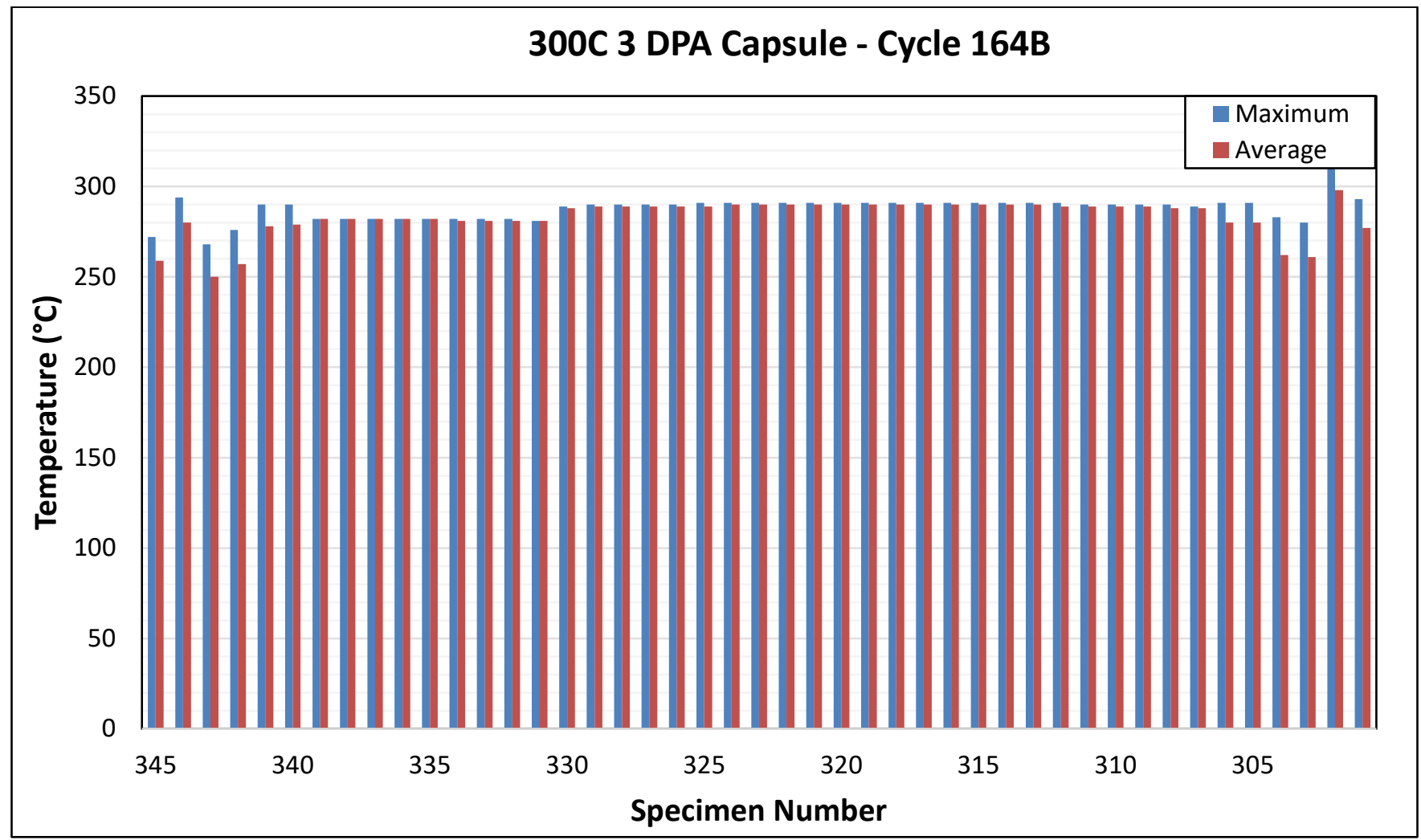

Figure 8-3: Specimen Temperatures for Capsule 300C 3 DPA in Cycle 164B 


\begin{tabular}{|cc|}
\hline $\begin{array}{c}\text { TEM-10200-1, Rev. } 11 \\
11 / 20 / 2019\end{array}$ & ECAR- 5510, Rev. 0 \\
& Page 12 of 29 \\
\hline
\end{tabular}

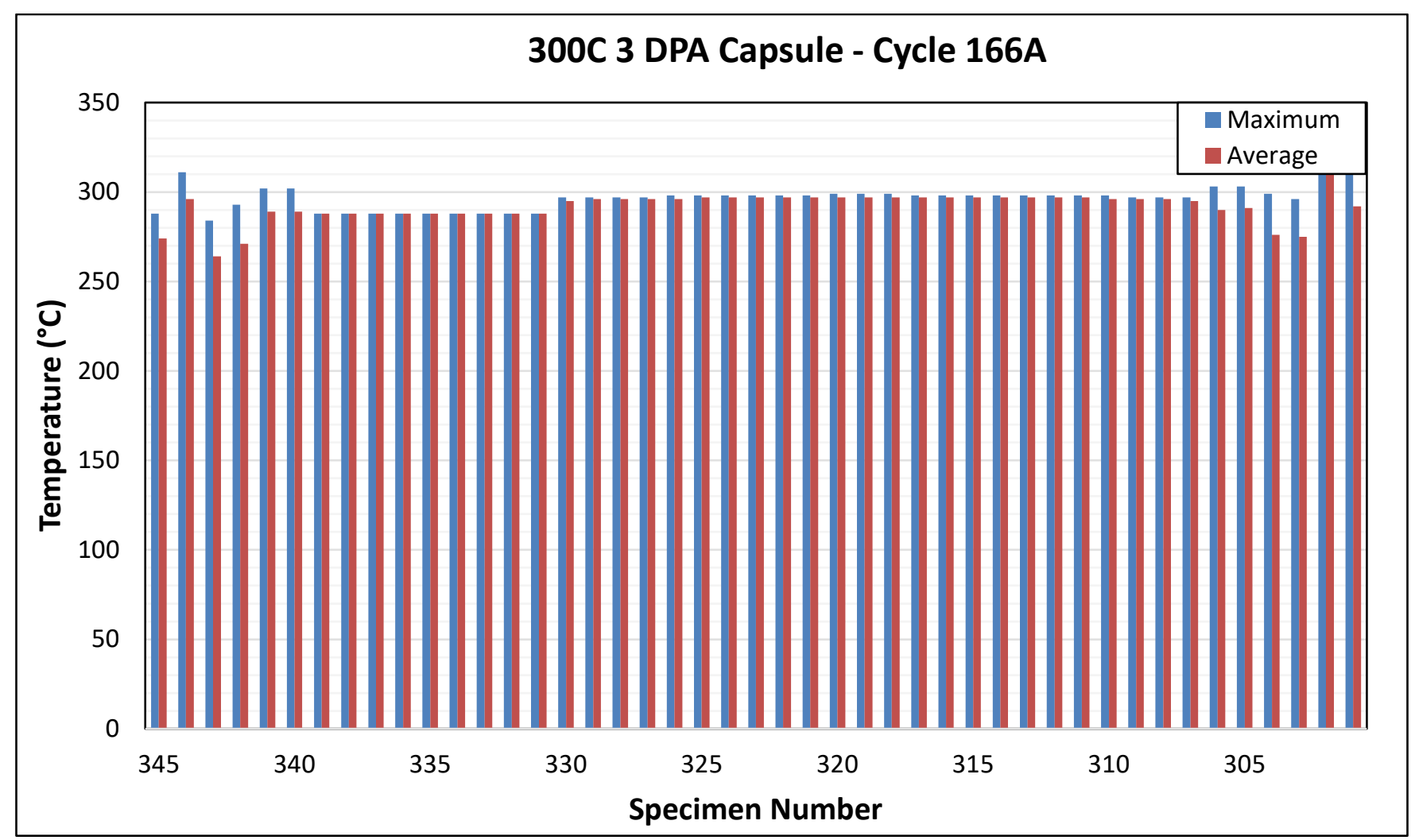

Figure 8-4: Specimen Temperatures for Capsule 300C 3 DPA in Cycle 166A

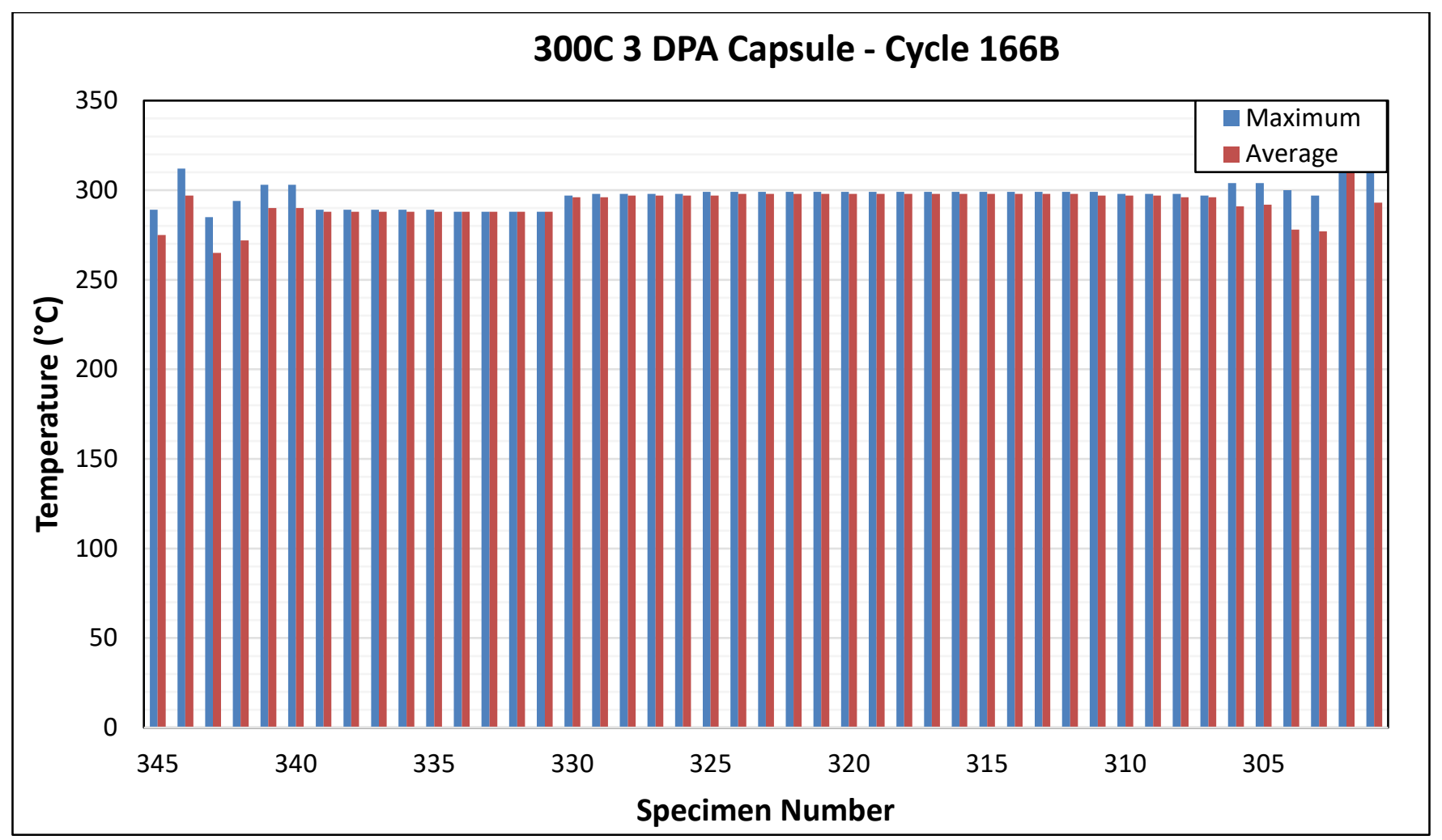

Figure 8-5: Specimen Temperatures for Capsule 300C 3 DPA in Cycle 166B 
BSU-8242 As-Run Thermal Analysis

Table 8-3: Specimen Temperatures for Capsule 300C 3 DPA in Cycles 164B, 166A, and 166B

\begin{tabular}{|c|c|c|c|c|c|c|c|}
\hline \multirow[t]{2}{*}{ Specimen/Material } & \multirow[t]{2}{*}{$\begin{array}{c}\text { Specimen } \\
\text { Number }\end{array}$} & \multicolumn{2}{|c|}{$\begin{array}{c}\text { Cycle 164B } \\
\text { Temperature } \\
{\left[{ }^{\circ} \mathrm{C}\right]}\end{array}$} & \multicolumn{2}{|c|}{$\begin{array}{c}\text { Cycle 166A } \\
\text { Temperature } \\
{\left[{ }^{\circ} \mathrm{C}\right]}\end{array}$} & \multicolumn{2}{|c|}{$\begin{array}{c}\text { Cycle 166B } \\
\text { Temperature } \\
{\left[{ }^{\circ} \mathrm{C}\right]}\end{array}$} \\
\hline & & Max & Avg & Max & Avg & Max & Avg \\
\hline RT-690 PM-HIP & 345 & 272 & 259 & 288 & 274 & 289 & 275 \\
\hline RT-690 PM-HIP & 344 & 294 & 280 & 311 & 296 & 312 & 297 \\
\hline RT-625 PM-HIP & 343 & 268 & 250 & 284 & 264 & 285 & 265 \\
\hline RT-625 PM-HIP & 342 & 276 & 257 & 293 & 271 & 294 & 272 \\
\hline CT-625 PM-HIP & 341 & 290 & 278 & 302 & 289 & 303 & 290 \\
\hline CT-625 PM-HIP & 340 & 290 & 279 & 302 & 289 & 303 & 290 \\
\hline TEM-USU-300-3-20 & 339 & 282 & 282 & 288 & 288 & 289 & 288 \\
\hline TEM-USU-300-3-20 & 338 & 282 & 282 & 288 & 288 & 289 & 288 \\
\hline TEM-USU-300-3-20 & 337 & 282 & 282 & 288 & 288 & 289 & 288 \\
\hline TEM-USU-300-3-28 & 336 & 282 & 282 & 288 & 288 & 289 & 288 \\
\hline TEM-USU-300-3-28 & 335 & 282 & 282 & 288 & 288 & 289 & 288 \\
\hline TEM-USU-300-3-28 & 334 & 282 & 281 & 288 & 288 & 288 & 288 \\
\hline TEM-USU-300-3-36 & 333 & 282 & 281 & 288 & 288 & 288 & 288 \\
\hline TEM-USU-300-3-36 & 332 & 282 & 281 & 288 & 288 & 288 & 288 \\
\hline TEM-USU-300-3-36 & 331 & 281 & 281 & 288 & 288 & 288 & 288 \\
\hline TEM-690 Forged & 330 & 289 & 288 & 297 & 295 & 297 & 296 \\
\hline TEM-690 Forged & 329 & 290 & 289 & 297 & 296 & 298 & 296 \\
\hline TEM-690 Forged & 328 & 290 & 289 & 297 & 296 & 298 & 297 \\
\hline TEM-690 Forged & 327 & 290 & 289 & 297 & 296 & 298 & 297 \\
\hline TEM-690 Forged & 326 & 290 & 289 & 298 & 296 & 298 & 297 \\
\hline TEM-690 Forged & 325 & 291 & 289 & 298 & 297 & 299 & 297 \\
\hline TEM-625 Forged & 324 & 291 & 290 & 298 & 297 & 299 & 298 \\
\hline TEM-625 Forged & 323 & 291 & 290 & 298 & 297 & 299 & 298 \\
\hline TEM-625 Forged & 322 & 291 & 290 & 298 & 297 & 299 & 298 \\
\hline TEM-625 Forged & 321 & 291 & 290 & 298 & 297 & 299 & 298 \\
\hline TEM-625 Forged & 320 & 291 & 290 & 299 & 297 & 299 & 298 \\
\hline TEM-625 Forged & 319 & 291 & 290 & 299 & 297 & 299 & 298 \\
\hline TEM-690 PM-HIP & 318 & 291 & 290 & 299 & 297 & 299 & 298 \\
\hline TEM-690 PM-HIP & 317 & 291 & 290 & 298 & 297 & 299 & 298 \\
\hline TEM-690 PM-HIP & 316 & 291 & 290 & 298 & 297 & 299 & 298 \\
\hline TEM-690 PM-HIP & 315 & 291 & 290 & 298 & 297 & 299 & 298 \\
\hline TEM-690 PM-HIP & 314 & 291 & 290 & 298 & 297 & 299 & 298 \\
\hline TEM-690 PM-HIP & 313 & 291 & 290 & 298 & 297 & 299 & 298 \\
\hline TEM-625 PM-HIP & 312 & 291 & 289 & 298 & 297 & 299 & 298 \\
\hline TEM-625 PM-HIP & 311 & 290 & 289 & 298 & 297 & 299 & 297 \\
\hline TEM-625 PM-HIP & 310 & 290 & 289 & 298 & 296 & 298 & 297 \\
\hline
\end{tabular}


BSU-8242 As-Run Thermal Analysis

\begin{tabular}{|c|c|c|c|c|c|c|c|}
\hline \multirow{2}{*}{ Specimen/Material } & $\begin{array}{c}\text { Specimen } \\
\text { Number }\end{array}$ & \multicolumn{2}{|c|}{$\begin{array}{c}\text { Cycle 164B } \\
\text { Temperature } \\
{\left[{ }^{\circ} \mathbf{C}\right]}\end{array}$} & \multicolumn{2}{c|}{$\begin{array}{c}\text { Cycle 166A } \\
\text { Temperature } \\
{\left[{ }^{\circ} \mathbf{C}\right]}\end{array}$} & \multicolumn{2}{c|}{$\begin{array}{c}\text { Cycle 166B } \\
\text { Temperature } \\
{\left[{ }^{\circ} \mathbf{C}\right]}\end{array}$} \\
\cline { 3 - 8 } & & Max & Avg & Max & Avg & Max & Avg \\
\hline TEM-625 PM-HIP & 309 & 290 & 289 & 297 & 296 & 298 & 297 \\
\hline TEM-625 PM-HIP & 308 & 290 & 288 & 297 & 296 & 298 & 296 \\
\hline TEM-625 PM-HIP & 307 & 289 & 288 & 297 & 295 & 297 & 296 \\
\hline CT-625 Cast & 306 & 291 & 280 & 303 & 290 & 304 & 291 \\
\hline CT-625 Cast & 305 & 291 & 280 & 303 & 291 & 304 & 292 \\
\hline RT-625 Forged & 304 & 283 & 262 & 299 & 276 & 300 & 278 \\
\hline RT-625 Forged & 303 & 280 & 261 & 296 & 275 & 297 & 277 \\
\hline RT-690 Forged & 302 & 312 & 298 & 329 & 315 & 331 & 316 \\
\hline RT-690 Forged & 301 & 293 & 277 & 310 & 292 & 311 & 293 \\
\hline
\end{tabular}

\subsubsection{C 1 DPA X Results}

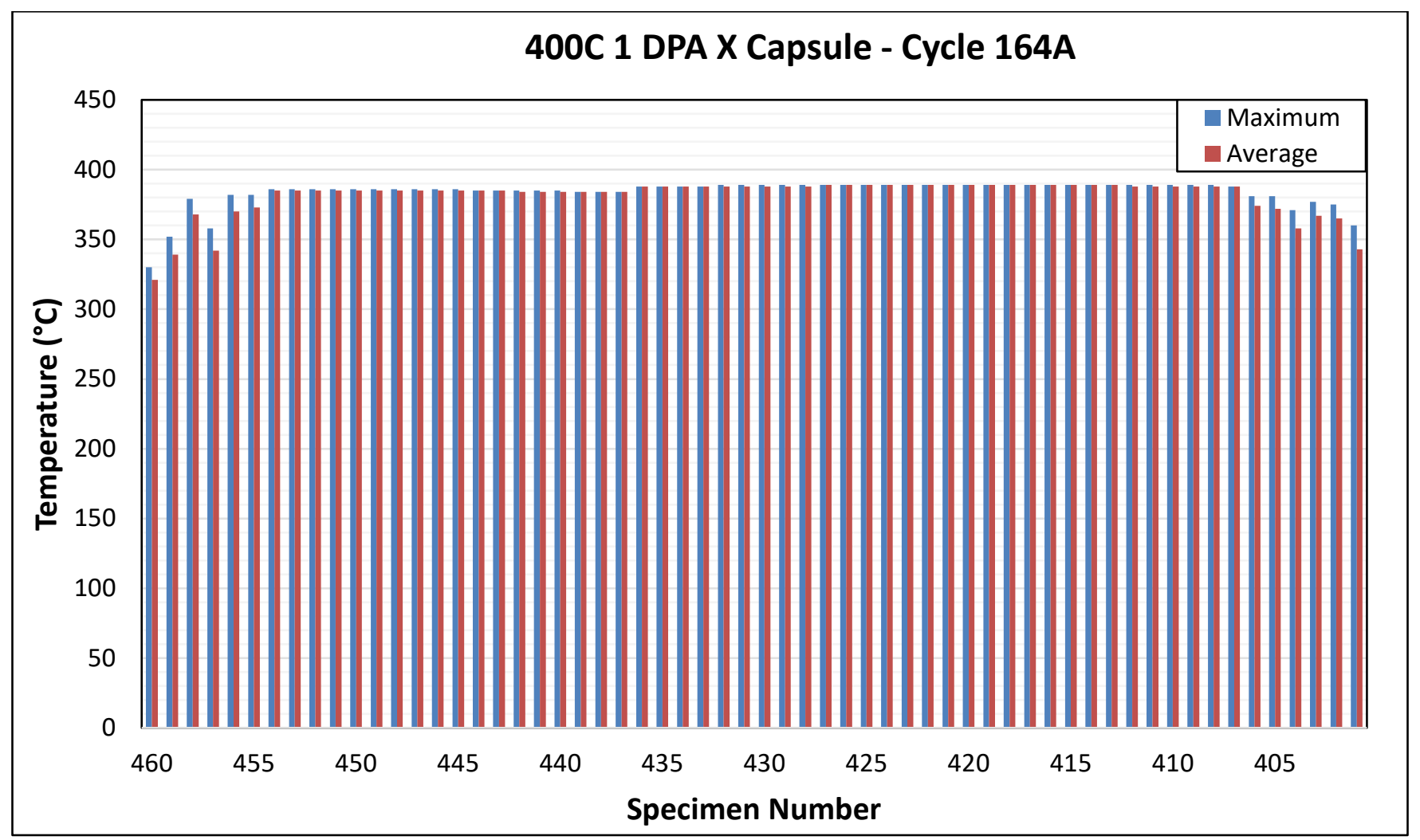

Figure 8-6: Specimen Temperatures for Capsule 400C 1 DPA X in Cycle 164A 
BSU-8242 As-Run Thermal Analysis

Table 8-4: Specimen Temperatures for Capsule 400C 1 DPA X in Cycle 164A

\begin{tabular}{|c|c|c|c|}
\hline \multirow[t]{2}{*}{ Specimen/Material } & \multirow[t]{2}{*}{$\begin{array}{c}\text { Specimen } \\
\text { Number }\end{array}$} & \multicolumn{2}{|c|}{$\begin{array}{c}\text { Cycle 164A } \\
\text { Temperature } \\
{\left[{ }^{\circ} \mathrm{C}\right]}\end{array}$} \\
\hline & & Max & Avg \\
\hline RT-625 PM-HIP & 460 & 330 & 321 \\
\hline RT-625 PM-HIP & 459 & 352 & 339 \\
\hline RT-690 PM-HIP & 458 & 379 & 368 \\
\hline RT-690 PM-HIP & 457 & 358 & 342 \\
\hline CT-625 PM-HIP & 456 & 382 & 370 \\
\hline CT-625 Cast & 455 & 382 & 373 \\
\hline TEM-625 PM-HIP & 454 & 386 & 385 \\
\hline TEM-625 PM-HIP & 453 & 386 & 385 \\
\hline TEM-625 PM-HIP & 452 & 386 & 385 \\
\hline TEM-625 PM-HIP & 451 & 386 & 385 \\
\hline TEM-625 PM-HIP & 450 & 386 & 385 \\
\hline TEM-625 PM-HIP & 449 & 386 & 385 \\
\hline TEM-625 Forged & 448 & 386 & 385 \\
\hline TEM-625 Forged & 447 & 386 & 385 \\
\hline TEM-625 Forged & 446 & 386 & 385 \\
\hline TEM-625 Forged & 445 & 386 & 385 \\
\hline TEM-625 Forged & 444 & 385 & 385 \\
\hline TEM-625 Forged & 443 & 385 & 385 \\
\hline $\begin{array}{l}\text { TEM-SA508 } \\
\text { Forged }\end{array}$ & 442 & 385 & 384 \\
\hline $\begin{array}{l}\text { TEM-SA508 } \\
\text { Forged }\end{array}$ & 441 & 385 & 384 \\
\hline $\begin{array}{l}\text { TEM-SA508 } \\
\text { Forged }\end{array}$ & 440 & 385 & 384 \\
\hline $\begin{array}{l}\text { TEM-SA508 } \\
\text { Forged }\end{array}$ & 439 & 384 & 384 \\
\hline $\begin{array}{l}\text { TEM-SA508 } \\
\text { Forged }\end{array}$ & 438 & 384 & 384 \\
\hline $\begin{array}{l}\text { TEM-SA508 } \\
\text { Forged }\end{array}$ & 437 & 384 & 384 \\
\hline $\begin{array}{c}\text { TEM-SA508 PM- } \\
\text { HIP }\end{array}$ & 436 & 388 & 388 \\
\hline $\begin{array}{c}\text { TEM-SA508 PM- } \\
\text { HIP }\end{array}$ & 435 & 388 & 388 \\
\hline $\begin{array}{c}\text { TEM-SA508 PM- } \\
\text { HIP }\end{array}$ & 434 & 388 & 388 \\
\hline $\begin{array}{c}\text { TEM-SA508 PM- } \\
\text { HIP }\end{array}$ & 433 & 388 & 388 \\
\hline $\begin{array}{c}\text { TEM-SA508 PM- } \\
\text { HIP }\end{array}$ & 432 & 389 & 388 \\
\hline
\end{tabular}




\begin{tabular}{|c|c|c|c|}
\hline \multirow{2}{*}{$\begin{array}{c}\text { Specimen/Material } \\
\text { Specimen } \\
\text { Number }\end{array}$} & \multicolumn{2}{|c|}{$\begin{array}{c}\text { Cycle 164A } \\
\text { Temperature } \\
\text { [ }\end{array}$} & $\begin{array}{c}{ }^{\circ} \text { ] } \\
\text { Max }\end{array}$ \\
\hline $\begin{array}{c}\text { TEM-SA508 PM- } \\
\text { HIP }\end{array}$ & 431 & 389 & 388 \\
\hline TEM-91 Cast & 430 & 389 & 388 \\
\hline TEM-91 Cast & 429 & 389 & 388 \\
\hline TEM-91 Cast & 428 & 389 & 388 \\
\hline TEM-91 Cast & 427 & 389 & 389 \\
\hline TEM-91 Cast & 426 & 389 & 389 \\
\hline TEM-91 Cast & 425 & 389 & 389 \\
\hline TEM-91 PM-HIP & 424 & 389 & 389 \\
\hline TEM-91 PM-HIP & 423 & 389 & 389 \\
\hline TEM-91 PM-HIP & 422 & 389 & 389 \\
\hline TEM-91 PM-HIP & 421 & 389 & 389 \\
\hline TEM-91 PM-HIP & 420 & 389 & 389 \\
\hline TEM-91 PM-HIP & 419 & 389 & 389 \\
\hline TEM-690 Forged & 418 & 389 & 389 \\
\hline TEM-690 Forged & 417 & 389 & 389 \\
\hline TEM-690 Forged & 416 & 389 & 389 \\
\hline TEM-690 Forged & 415 & 389 & 389 \\
\hline TEM-690 Forged & 414 & 389 & 389 \\
\hline TEM-690 Forged & 413 & 389 & 389 \\
\hline TEM-690 PM-HIP & 412 & 389 & 388 \\
\hline TEM-690 PM-HIP & 411 & 389 & 388 \\
\hline TEM-690 PM-HIP & 410 & 389 & 388 \\
\hline TEM-690 PM-HIP & 409 & 389 & 388 \\
\hline TEM-690 PM-HIP & 408 & 389 & 388 \\
\hline TEM-690 PM-HIP & 407 & 388 & 388 \\
\hline CT-690 PM-HIP & 406 & 381 & 374 \\
\hline CT-690 PM-HIP & 405 & 381 & 372 \\
\hline RT-91 PM-HIP & 404 & 371 & 358 \\
\hline RT-91 PM-HIP & 403 & 377 & 367 \\
\hline RT-SA508 PM-HIP & 402 & 360 & 343 \\
\hline RT-SA508 PM-HIP & 401 & & \\
\hline
\end{tabular}




\subsubsection{C 1 DPA Y Results}

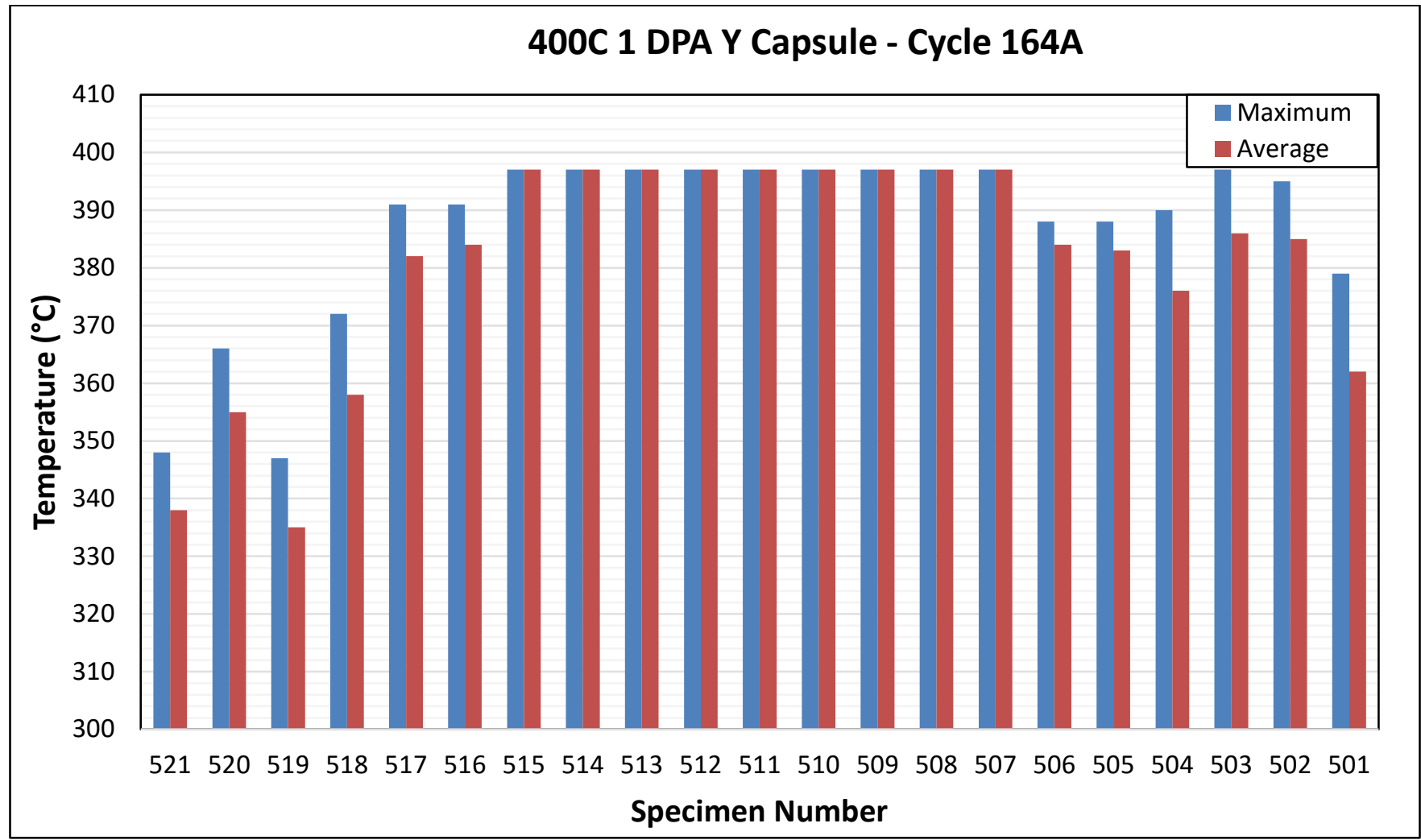

Figure 8-7: Specimen Temperatures for Capsule 400C 1 DPA Y in Cycle 164A

Table 8-5: Specimen Temperatures for Capsule 400C 1 DPA Y in Cycle 164A

\begin{tabular}{|c|c|c|c|}
\hline \multirow{2}{*}{ Specimen/Material } & $\begin{array}{c}\text { Specimen } \\
\text { Number }\end{array}$ & \multicolumn{2}{|c|}{$\begin{array}{c}\text { Cycle 164A } \\
\text { Temperature } \\
{\left[{ }^{\circ} \mathrm{C}\right]}\end{array}$} \\
\cline { 3 - 4 } & & Max & Avg \\
\hline RT-625 Forged & 521 & 348 & 338 \\
\hline RT-625 Forged & 520 & 366 & 355 \\
\hline RT-690 Forged & 519 & 347 & 335 \\
\hline RT-690 Forged & 518 & 372 & 358 \\
\hline CT-690 Forged & 517 & 391 & 382 \\
\hline CT-690 Forged & 516 & 391 & 384 \\
\hline $\begin{array}{c}\text { TEM-USU-400-1- } \\
20\end{array}$ & 515 & 397 & 397 \\
\hline $\begin{array}{c}\text { TEM-USU-400-1- } \\
20\end{array}$ & 514 & 397 & 397 \\
\hline $\begin{array}{c}\text { TEM-USU-400-1- } \\
20\end{array}$ & 513 & 397 & 397 \\
\hline $\begin{array}{c}\text { TEM-USU-400-1- } \\
28\end{array}$ & 512 & 397 & 397 \\
\hline
\end{tabular}




\begin{tabular}{|c|c|c|c|}
\hline $\begin{array}{l}\text { TEM-10200-1, Rev. } 11 \\
\text { 11/20/2019 }\end{array}$ & \multicolumn{3}{|c|}{$\begin{array}{l}\text { ENGINEERING CALCULATIONS AND } \\
\text { BSU-8242 As-Run Thermal }\end{array}$} \\
\hline \multirow[t]{2}{*}{ Specimen/Material } & \multirow[t]{2}{*}{$\begin{array}{l}\text { Specimen } \\
\text { Number }\end{array}$} & \multicolumn{2}{|c|}{$\begin{array}{c}\text { Cycle 164A } \\
\text { Temperature } \\
{\left[{ }^{\circ} \mathrm{C}\right]}\end{array}$} \\
\hline & & Max & Avg \\
\hline $\begin{array}{c}\text { TEM-USU-400-1- } \\
28\end{array}$ & 511 & 397 & 397 \\
\hline $\begin{array}{c}\text { TEM-USU-400-1- } \\
28\end{array}$ & 510 & 397 & 397 \\
\hline $\begin{array}{c}\text { TEM-USU-400-1- } \\
36\end{array}$ & 509 & 397 & 397 \\
\hline $\begin{array}{l}\text { TEM-USU-400-1- } \\
36\end{array}$ & 508 & 397 & 397 \\
\hline $\begin{array}{l}\text { TEM-USU-400-1- } \\
36 \\
\end{array}$ & 507 & 397 & 397 \\
\hline CT-91 PM-HIP & 506 & 388 & 384 \\
\hline CT-91 PM-HIP & 505 & 388 & 383 \\
\hline RT-91 Cast & 504 & 390 & 376 \\
\hline RT-91 Cast & 503 & 397 & 386 \\
\hline RT-SA508 Forged & 502 & 395 & 385 \\
\hline RT-SA508 Forged & 501 & 379 & 362 \\
\hline
\end{tabular}

\subsubsection{C 3 DPA X Results}

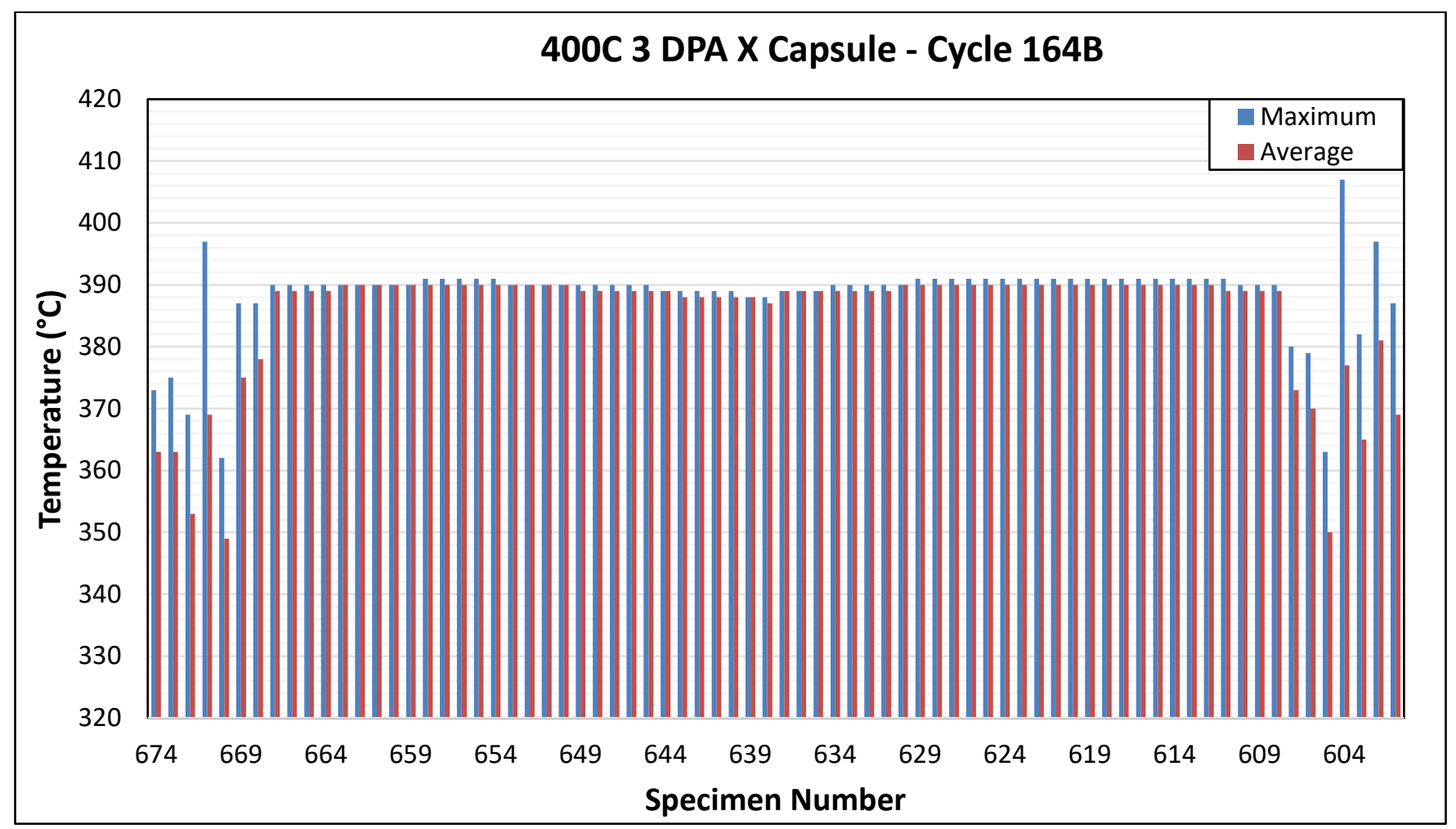

Figure 8-8: Specimen Temperatures for Capsule 400C 3 DPA X in Cycle 164B 


\begin{tabular}{|cc|}
\hline $\begin{array}{cc}\text { TEM-10200-1, Rev. } 11 & \text { ENGINEERING CALCULATIONS AND ANALYSIS } \\
11 / 20 / 2019 & \text { Page } 19 \text { of } 29 \\
& \text { BSU-8242 As-Run Thermal Analysis }\end{array}$ \\
\hline
\end{tabular}

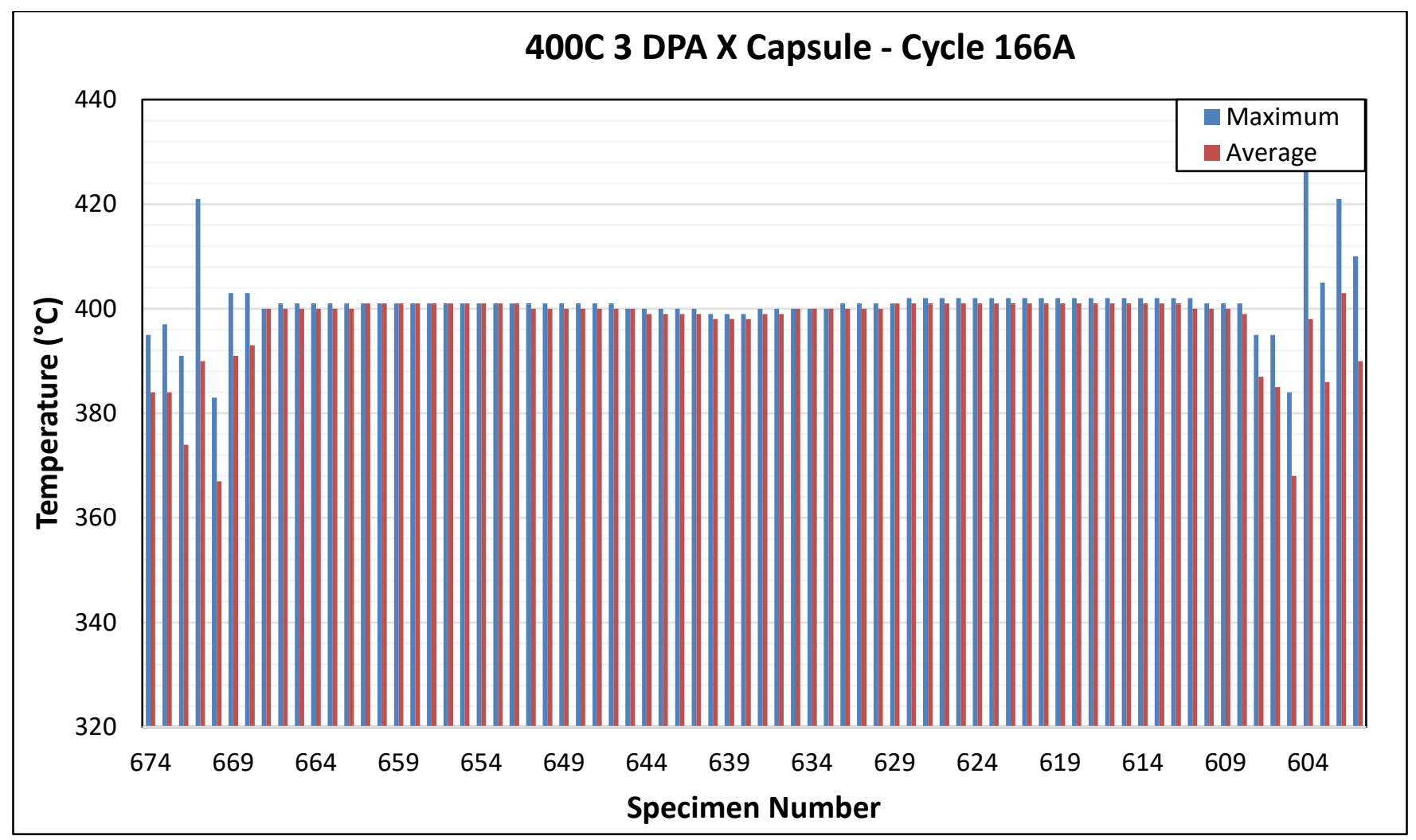

Figure 8-9: Specimen Temperatures for Capsule 400C 3 DPA X in Cycle 166A

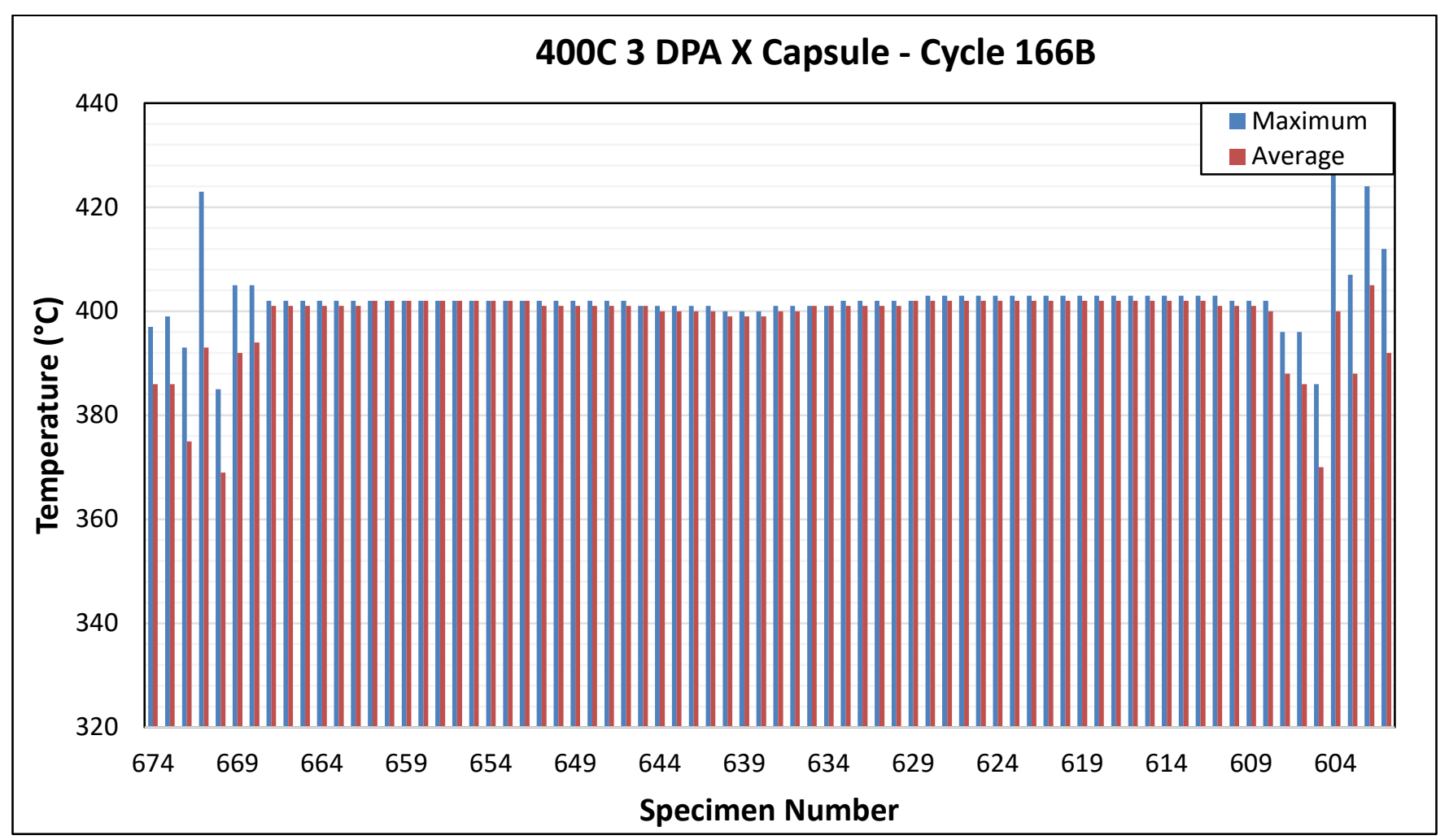

Figure 8-10: Specimen Temperatures for Capsule 400C 3 DPA X in Cycle 166B 
BSU-8242 As-Run Thermal Analysis

Table 8-6: Specimen Temperatures for Capsule 400C 3 DPA X in Cycles 164B, 166A, and 166B

\begin{tabular}{|c|c|c|c|c|c|c|c|}
\hline \multirow[t]{2}{*}{ Specimen/Material } & \multirow[t]{2}{*}{$\begin{array}{c}\text { Specimen } \\
\text { Number }\end{array}$} & \multicolumn{2}{|c|}{$\begin{array}{c}\text { Cycle 164B } \\
\text { Temperature } \\
{\left[{ }^{\circ} \mathrm{C}\right]}\end{array}$} & \multicolumn{2}{|c|}{$\begin{array}{c}\text { Cycle 166A } \\
\text { Temperature } \\
{\left[{ }^{\circ} \mathrm{C}\right]}\end{array}$} & \multicolumn{2}{|c|}{$\begin{array}{c}\text { Cycle 166B } \\
\text { Temperature } \\
{\left[{ }^{\circ} \mathrm{C}\right]}\end{array}$} \\
\hline & & Max & Avg & Max & Avg & Max & Avg \\
\hline RT-690 PM-HIP & 674 & 373 & 363 & 395 & 384 & 397 & 386 \\
\hline RT-690 PM-HIP & 673 & 375 & 363 & 397 & 384 & 399 & 386 \\
\hline RT-625 PM-HIP & 672 & 369 & 353 & 391 & 374 & 393 & 375 \\
\hline RT-625 PM-HIP & 671 & 397 & 369 & 421 & 390 & 423 & 393 \\
\hline RT-91 PM-HIP & 670 & 362 & 349 & 383 & 367 & 385 & 369 \\
\hline CT-625 PM-HIP & 669 & 387 & 375 & 403 & 391 & 405 & 392 \\
\hline CT-625 Cast & 668 & 387 & 378 & 403 & 393 & 405 & 394 \\
\hline TEM-91 Cast & 667 & 390 & 389 & 400 & 400 & 402 & 401 \\
\hline TEM-91 Cast & 666 & 390 & 389 & 401 & 400 & 402 & 401 \\
\hline TEM-91 Cast & 665 & 390 & 389 & 401 & 400 & 402 & 401 \\
\hline TEM-91 Cast & 664 & 390 & 389 & 401 & 400 & 402 & 401 \\
\hline TEM-91 Cast & 663 & 390 & 390 & 401 & 400 & 402 & 401 \\
\hline TEM-91 Cast & 662 & 390 & 390 & 401 & 400 & 402 & 401 \\
\hline TEM-304L Forged & 661 & 390 & 390 & 401 & 401 & 402 & 402 \\
\hline TEM-304L Forged & 660 & 390 & 390 & 401 & 401 & 402 & 402 \\
\hline TEM-304L Forged & 659 & 390 & 390 & 401 & 401 & 402 & 402 \\
\hline TEM-304L Forged & 658 & 391 & 390 & 401 & 401 & 402 & 402 \\
\hline TEM-304L Forged & 657 & 391 & 390 & 401 & 401 & 402 & 402 \\
\hline TEM-304L Forged & 656 & 391 & 390 & 401 & 401 & 402 & 402 \\
\hline TEM-304L Forged & 655 & 391 & 390 & 401 & 401 & 402 & 402 \\
\hline TEM-304L Forged & 654 & 391 & 390 & 401 & 401 & 402 & 402 \\
\hline TEM-304L Forged & 653 & 390 & 390 & 401 & 401 & 402 & 402 \\
\hline TEM-304L Forged & 652 & 390 & 390 & 401 & 401 & 402 & 402 \\
\hline TEM-304L Forged & 651 & 390 & 390 & 401 & 400 & 402 & 401 \\
\hline TEM-304L Forged & 650 & 390 & 390 & 401 & 400 & 402 & 401 \\
\hline TEM-316L PM-HIP & 649 & 390 & 389 & 401 & 400 & 402 & 401 \\
\hline TEM-316L PM-HIP & 648 & 390 & 389 & 401 & 400 & 402 & 401 \\
\hline TEM-316L PM-HIP & 647 & 390 & 389 & 401 & 400 & 402 & 401 \\
\hline TEM-316L PM-HIP & 646 & 390 & 389 & 401 & 400 & 402 & 401 \\
\hline TEM-316L PM-HIP & 645 & 390 & 389 & 400 & 400 & 401 & 401 \\
\hline TEM-316L PM-HIP & 644 & 389 & 389 & 400 & 399 & 401 & 400 \\
\hline TEM-316L Forged & 643 & 389 & 388 & 400 & 399 & 401 & 400 \\
\hline TEM-316L Forged & 642 & 389 & 388 & 400 & 399 & 401 & 400 \\
\hline TEM-316L Forged & 641 & 389 & 388 & 400 & 399 & 401 & 400 \\
\hline TEM-316L Forged & 640 & 389 & 388 & 399 & 398 & 400 & 399 \\
\hline TEM-316L Forged & 639 & 388 & 388 & 399 & 398 & 400 & 399 \\
\hline
\end{tabular}


BSU-8242 As-Run Thermal Analysis

\begin{tabular}{|c|c|c|c|c|c|c|c|}
\hline \multirow[t]{2}{*}{ Specimen/Material } & \multirow[t]{2}{*}{$\begin{array}{c}\text { Specimen } \\
\text { Number }\end{array}$} & \multicolumn{2}{|c|}{$\begin{array}{c}\text { Cycle 164B } \\
\text { Temperature } \\
{\left[{ }^{\circ} \mathrm{C}\right]}\end{array}$} & \multicolumn{2}{|c|}{$\begin{array}{c}\text { Cycle 166A } \\
\text { Temperature } \\
{\left[{ }^{\circ} \mathrm{C}\right]}\end{array}$} & \multicolumn{2}{|c|}{$\begin{array}{c}\text { Cycle 166B } \\
\text { Temperature } \\
{\left[{ }^{\circ} \mathrm{C}\right]}\end{array}$} \\
\hline & & Max & Avg & Max & Avg & Max & Avg \\
\hline TEM-316L Forged & 638 & 388 & 387 & 399 & 398 & 400 & 399 \\
\hline TEM-91 PM-HIP & 637 & 389 & 389 & 400 & 399 & 401 & 400 \\
\hline TEM-91 PM-HIP & 636 & 389 & 389 & 400 & 399 & 401 & 400 \\
\hline TEM-91 PM-HIP & 635 & 389 & 389 & 400 & 400 & 401 & 401 \\
\hline TEM-91 PM-HIP & 634 & 390 & 389 & 400 & 400 & 401 & 401 \\
\hline TEM-91 PM-HIP & 633 & 390 & 389 & 400 & 400 & 402 & 401 \\
\hline TEM-91 PM-HIP & 632 & 390 & 389 & 401 & 400 & 402 & 401 \\
\hline TEM-690 Forged & 631 & 390 & 389 & 401 & 400 & 402 & 401 \\
\hline TEM-690 Forged & 630 & 390 & 390 & 401 & 400 & 402 & 401 \\
\hline TEM-690 Forged & 629 & 391 & 390 & 401 & 401 & 402 & 402 \\
\hline TEM-690 Forged & 628 & 391 & 390 & 402 & 401 & 403 & 402 \\
\hline TEM-690 Forged & 627 & 391 & 390 & 402 & 401 & 403 & 402 \\
\hline TEM-690 Forged & 626 & 391 & 390 & 402 & 401 & 403 & 402 \\
\hline TEM-690 PM-HIP & 625 & 391 & 390 & 402 & 401 & 403 & 402 \\
\hline TEM-690 PM-HIP & 624 & 391 & 390 & 402 & 401 & 403 & 402 \\
\hline TEM-690 PM-HIP & 623 & 391 & 390 & 402 & 401 & 403 & 402 \\
\hline TEM-690 PM-HIP & 622 & 391 & 390 & 402 & 401 & 403 & 402 \\
\hline TEM-690 PM-HIP & 621 & 391 & 390 & 402 & 401 & 403 & 402 \\
\hline TEM-690 PM-HIP & 620 & 391 & 390 & 402 & 401 & 403 & 402 \\
\hline TEM-625 Forged & 619 & 391 & 390 & 402 & 401 & 403 & 402 \\
\hline TEM-625 Forged & 618 & 391 & 390 & 402 & 401 & 403 & 402 \\
\hline TEM-625 Forged & 617 & 391 & 390 & 402 & 401 & 403 & 402 \\
\hline TEM-625 Forged & 616 & 391 & 390 & 402 & 401 & 403 & 402 \\
\hline TEM-625 Forged & 615 & 391 & 390 & 402 & 401 & 403 & 402 \\
\hline TEM-625 Forged & 614 & 391 & 390 & 402 & 401 & 403 & 402 \\
\hline TEM-625 PM-HIP & 613 & 391 & 390 & 402 & 401 & 403 & 402 \\
\hline TEM-625 PM-HIP & 612 & 391 & 390 & 402 & 401 & 403 & 402 \\
\hline TEM-625 PM-HIP & 611 & 391 & 389 & 402 & 400 & 403 & 401 \\
\hline TEM-625 PM-HIP & 610 & 390 & 389 & 401 & 400 & 402 & 401 \\
\hline TEM-625 PM-HIP & 609 & 390 & 389 & 401 & 400 & 402 & 401 \\
\hline TEM-625 PM-HIP & 608 & 390 & 389 & 401 & 399 & 402 & 400 \\
\hline CT-690 PM-HIP & 607 & 380 & 373 & 395 & 387 & 396 & 388 \\
\hline CT-690 PM-HIP & 606 & 379 & 370 & 395 & 385 & 396 & 386 \\
\hline RT-91 Cast & 605 & 363 & 350 & 384 & 368 & 386 & 370 \\
\hline RT-625 Forged & 604 & 407 & 377 & 431 & 398 & 434 & 400 \\
\hline RT-625 Forged & 603 & 382 & 365 & 405 & 386 & 407 & 388 \\
\hline RT-690 Forged & 602 & 387 & 370 & 411 & 392 & 413 & 394 \\
\hline
\end{tabular}




\begin{tabular}{|c|c|c|c|c|c|c|c|}
\hline \multirow{2}{*}{ Specimen/Material } & $\begin{array}{c}\text { Specimen } \\
\text { Number }\end{array}$ & \multicolumn{2}{|c|}{$\begin{array}{c}\text { Cycle 164B } \\
\text { Temperature } \\
{\left[{ }^{\circ} \mathrm{C}\right]}\end{array}$} & $\begin{array}{c}\text { Cycle 166A } \\
\text { Temperature } \\
{\left[{ }^{\circ} \mathrm{C}\right]}\end{array}$ & $\begin{array}{c}\text { Cycle 166B } \\
\text { Temperature } \\
{\left[{ }^{\circ} \mathrm{C}\right]}\end{array}$ \\
\cline { 3 - 8 } & & $\operatorname{Max}$ & Avg & Max & Avg & Max & Avg \\
\hline RT-690 Forged & 601 & 376 & 358 & 400 & 380 & 402 & 382 \\
\hline
\end{tabular}

\subsubsection{C 3 DPA Y Results}

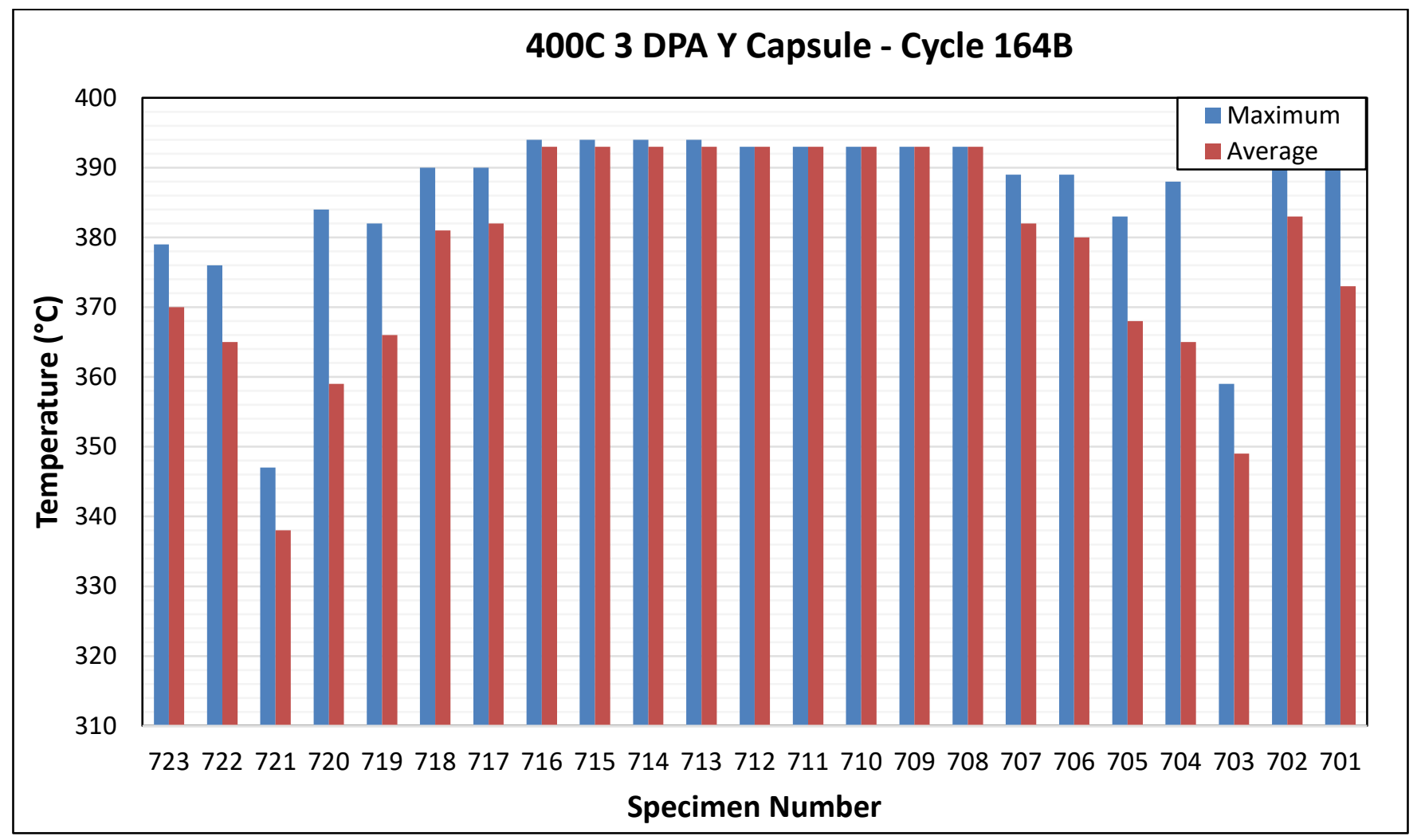

Figure 8-11: Specimen Temperatures for Capsule 400C 3 DPA Y in Cycle 164B 


\begin{tabular}{|cc|}
\hline $\begin{array}{c}\text { TEM-10200-1, Rev. } 11 \\
11 / 20 / 2019\end{array}$ & ECAR- 5510, Rev. 0 \\
Page 23 of 29 \\
BSU-8242 As-Run Thermal Analysis
\end{tabular}

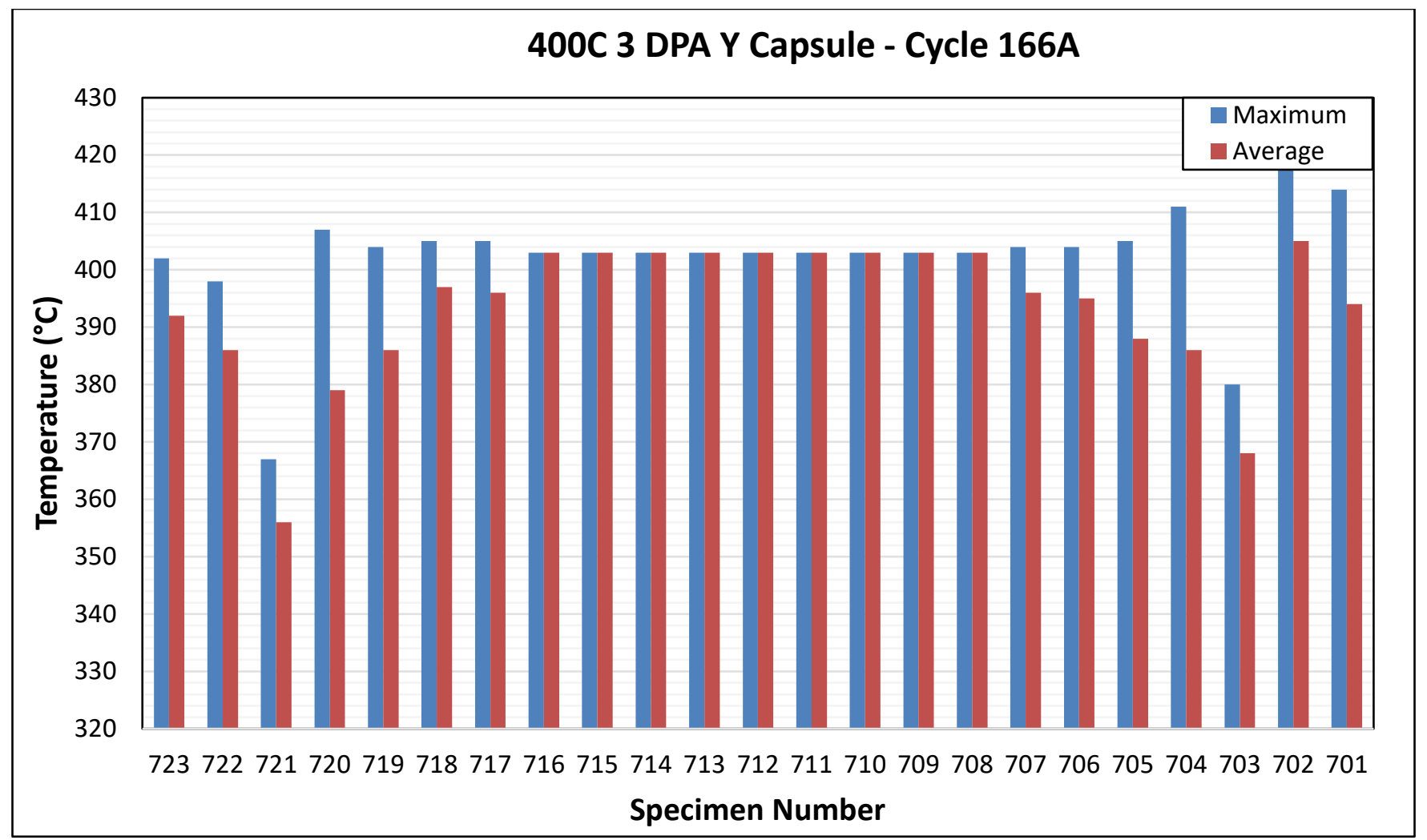

Figure 8-12: Specimen Temperatures for Capsule 400C 3 DPA Y in Cycle 166A

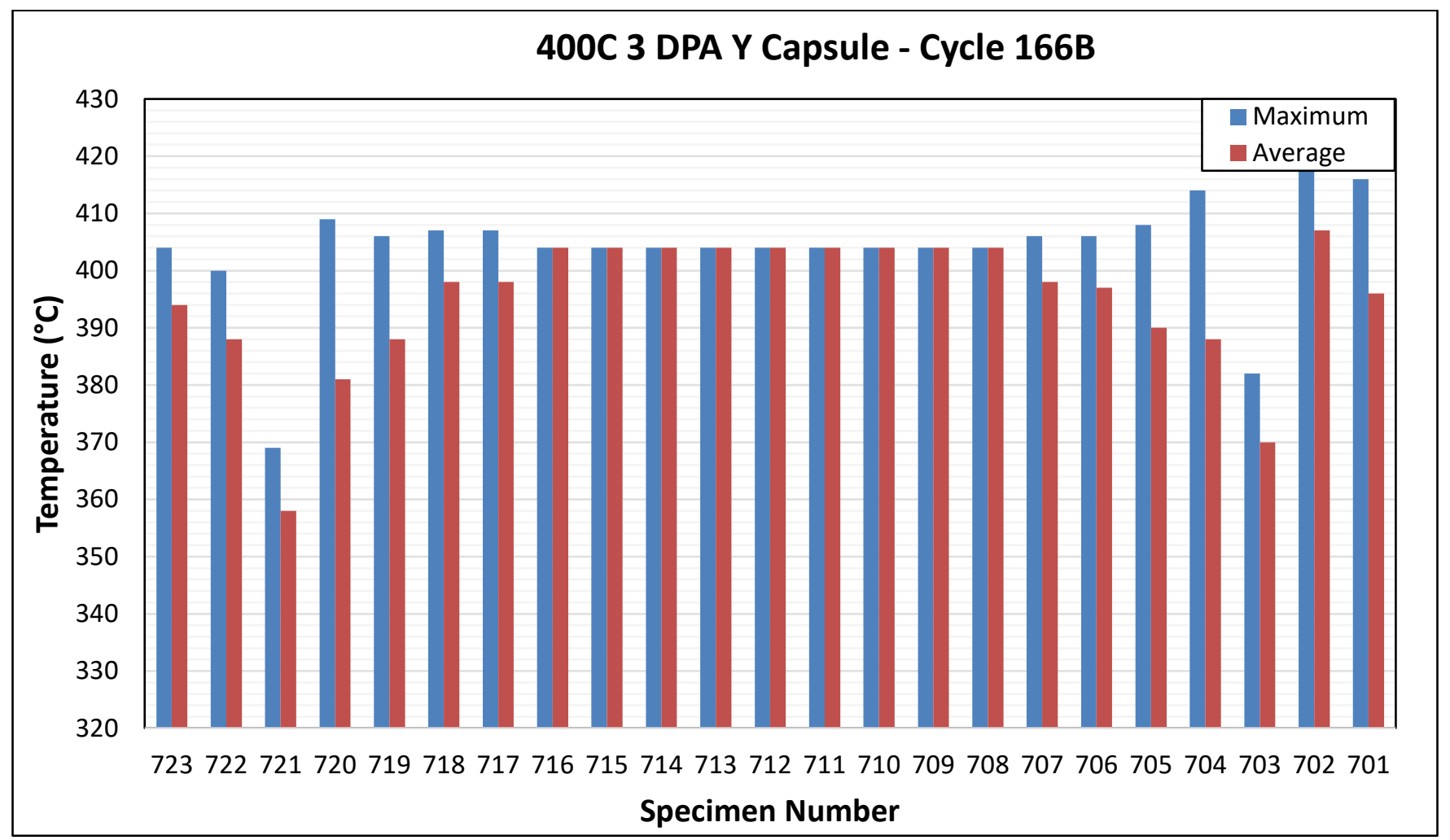

Figure 8-13: Specimen Temperatures for Capsule 400C 3 DPA Y in Cycle 166B 
BSU-8242 As-Run Thermal Analysis

Table 8-7: Specimen Temperatures for Capsule 400C 3 DPA Y in Cycle 164B, 166A, and 166B

\begin{tabular}{|c|c|c|c|c|c|c|c|}
\hline \multirow[t]{2}{*}{ Specimen/Material } & \multirow[t]{2}{*}{$\begin{array}{c}\text { Specimen } \\
\text { Number }\end{array}$} & \multicolumn{2}{|c|}{$\begin{array}{c}\text { Cycle 164B } \\
\text { Temperature } \\
{\left[{ }^{\circ} \mathrm{C}\right]}\end{array}$} & \multicolumn{2}{|c|}{$\begin{array}{c}\text { Cycle 166A } \\
\text { Temperature } \\
{\left[{ }^{\circ} \mathrm{C}\right]}\end{array}$} & \multicolumn{2}{|c|}{$\begin{array}{c}\text { Cycle 166B } \\
\text { Temperature } \\
{\left[{ }^{\circ} \mathrm{C}\right]}\end{array}$} \\
\hline & & Max & Avg & Max & Avg & Max & Avg \\
\hline RT-304L Forged & 723 & 379 & 370 & 402 & 392 & 404 & 394 \\
\hline RT-304L Forged & 722 & 376 & 365 & 398 & 386 & 400 & 388 \\
\hline RT-91 PM-HIP & 721 & 347 & 338 & 367 & 356 & 369 & 358 \\
\hline RT-316L PM-HIP & 720 & 384 & 359 & 407 & 379 & 409 & 381 \\
\hline RT-316L PM-HIP & 719 & 382 & 366 & 404 & 386 & 406 & 388 \\
\hline CT-690 Forged & 718 & 390 & 381 & 405 & 397 & 407 & 398 \\
\hline CT-316 Cast & 717 & 390 & 382 & 405 & 396 & 407 & 398 \\
\hline TEM-USU-400-3-36 & 716 & 394 & 393 & 403 & 403 & 404 & 404 \\
\hline TEM-USU-400-3-36 & 715 & 394 & 393 & 403 & 403 & 404 & 404 \\
\hline TEM-USU-400-3-36 & 714 & 394 & 393 & 403 & 403 & 404 & 404 \\
\hline TEM-USU-400-3-28 & 713 & 394 & 393 & 403 & 403 & 404 & 404 \\
\hline TEM-USU-400-3-28 & 712 & 393 & 393 & 403 & 403 & 404 & 404 \\
\hline TEM-USU-400-3-28 & 711 & 393 & 393 & 403 & 403 & 404 & 404 \\
\hline TEM-USU-400-3-20 & 710 & 393 & 393 & 403 & 403 & 404 & 404 \\
\hline TEM-USU-400-3-20 & 709 & 393 & 393 & 403 & 403 & 404 & 404 \\
\hline TEM-USU-400-3-20 & 708 & 393 & 393 & 403 & 403 & 404 & 404 \\
\hline CT-316 PM-HIP & 707 & 389 & 382 & 404 & 396 & 406 & 398 \\
\hline CT-316 PM-HIP & 706 & 389 & 380 & 404 & 395 & 406 & 397 \\
\hline RT-316L Forged & 705 & 383 & 368 & 405 & 388 & 408 & 390 \\
\hline RT-316L Forged & 704 & 388 & 365 & 411 & 386 & 414 & 388 \\
\hline RT-91 Cast & 703 & 359 & 349 & 380 & 368 & 382 & 370 \\
\hline RT-304L Forged & 702 & 394 & 383 & 418 & 405 & 420 & 407 \\
\hline RT-304L Forged & 701 & 391 & 373 & 414 & 394 & 416 & 396 \\
\hline
\end{tabular}

\subsection{Melt Wire Comparison}

At the time of this ECAR, the only melt wire evaluation that had been performed was on the $1 \mathrm{dpa}$ capsules. This evaluation can be found in INL EXT-20-58375 [4]. The following table shows a summary of the melt wire evaluation and was created using Table 1 in INL EXT-20-58375. 
BSU-8242 As-Run Thermal Analysis

Table 8-8: Melt Wire Evaluation Table From INL EXT-20-58375 Table 1

\begin{tabular}{|c|c|c|c|}
\hline $\begin{array}{c}\text { Melt Wire Pack Serial } \\
\text { Number }\end{array}$ & $\begin{array}{l}\text { Experiment/HFEF } \\
\text { Sample ID }\end{array}$ & $\begin{array}{c}\text { \% Weight Each Wire in } \\
\text { Package and (Melt } \\
\text { Temperature) }\end{array}$ & Temperature Range \\
\hline 400C-1DPA-X & BSU/MELTWRE4 & $\begin{array}{c}100 \mathrm{~Pb}\left(327.5^{\circ} \mathrm{C}\right), 85 \mathrm{Te} \\
15 \mathrm{Sn}\left(399.4^{\circ} \mathrm{C}\right), 100 \mathrm{Te} \\
\left(449.5^{\circ} \mathrm{C}\right) .\end{array}$ & $\begin{array}{c}327.5^{\circ} \mathrm{C}<\mathrm{T} \\
399.4^{\circ} \mathrm{C}\end{array}$ \\
\hline 300C-1DPA-X & BSU/MELTWRE1 & $\begin{array}{c}95 \mathrm{Sn} 5 \mathrm{Sb}\left(238.6^{\circ} \mathrm{C}\right) \\
100 \mathrm{Bi}\left(271.5^{\circ} \mathrm{C}\right), 100 \mathrm{~Pb} \\
\left(327.5^{\circ} \mathrm{C}\right)\end{array}$ & $\mathrm{T}<238.6^{\circ} \mathrm{C}$ \\
\hline 300C-1DPA-Y & BSU/MELTWRE2 & $\begin{array}{c}95 \mathrm{Sn} 5 \mathrm{Sb}\left(238.6^{\circ} \mathrm{C}\right) \\
100 \mathrm{Bi}\left(271.5^{\circ} \mathrm{C}\right), 100 \mathrm{~Pb} \\
\left(327.5^{\circ} \mathrm{C}\right) .\end{array}$ & $\begin{array}{l}238.6^{\circ} \mathrm{C}<\mathrm{T} \\
<271.5^{\circ} \mathrm{C}\end{array}$ \\
\hline 400C-1DPA-Y & BSU/MELTWRE5 & $\begin{array}{c}100 \mathrm{~Pb}\left(327.5^{\circ} \mathrm{C}\right), 85 \mathrm{Te} \\
15 \mathrm{Sn}\left(399.4^{\circ} \mathrm{C}\right), 100 \mathrm{Te} \\
\left(449.5^{\circ} \mathrm{C}\right) .\end{array}$ & $\begin{array}{c}399.4^{\circ} \mathrm{C}<\mathrm{T}< \\
449.5^{\circ} \mathrm{C}\end{array}$ \\
\hline
\end{tabular}

The following table (Table 8-9) is from ECAR-3575 [1] (Table 13) which shows what the melt wire temperature would be if the specimen temperatures were what was calculated in ECAR-3575. Because of the location of the melt wires, the temperature is slightly lower than what the specimens would see.

Table 8-9: Melt Wire Temperature Based on Specimen Temperatures Calculated in ECAR-3575

\begin{tabular}{|c|c|}
\hline Capsule & $\begin{array}{c}\text { Melt Wire } \\
\text { Temperature }\left[{ }^{\circ} \mathbf{C} \text { ] }\right.\end{array}$ \\
\hline 400C 1 dpa $X$ & 352 \\
\hline 300C 1 dpa X & 258 \\
\hline 300C 1 dpa $Y$ & 257 \\
\hline 400C 1 dpa $Y$ & 367 \\
\hline
\end{tabular}

Table 8-10: Summary of Average Calculated As-Run Temperatures

\begin{tabular}{|c|c|c|c|}
\hline \multirow{2}{*}{ Capsule } & \multicolumn{2}{|c|}{ Temperature $\left[{ }^{\circ} \mathrm{C}\right]$} & \multirow{2}{*}{$\begin{array}{l}\text { Temperature } \\
\text { Deviation from } \\
\text { Target }\left[{ }^{\circ} \mathrm{C}\right]\end{array}$} \\
\hline & Target & Average & \\
\hline 300C $1 \mathrm{dpa} X$ & 300 & 276 & -24 \\
\hline 300C 1 dpa Y & 300 & 272 & -28 \\
\hline 300C 3 dpa* & 300 & 287 & -13 \\
\hline 400C 1 dpa X & 400 & 362 & -38 \\
\hline 400C 1 dpa Y & 400 & 371 & -29 \\
\hline $400 C 3 \mathrm{dpa} X^{*}$ & 400 & 389 & -11 \\
\hline 400C 3 dpa $Y^{*}$ & 400 & 390 & -10 \\
\hline
\end{tabular}


The 400C $1 \mathrm{dpa} X$ melt wire pack showed that the experiments maximum melt wire temperature was between $327.5^{\circ} \mathrm{C}$ and $399.4^{\circ} \mathrm{C}$. According to the model, if the melt wire pack is at $352^{\circ} \mathrm{C}$ then the average specimen temperature is $397^{\circ} \mathrm{C}$ (information from ECAR-3575, Table 11). This means the actual temperature based on the melt wires is somewhere between $373^{\circ} \mathrm{C}$ and $444^{\circ} \mathrm{C}$. Comparing this to the calculated as-run temperatures, the average ${ }^{1}$ specimen temperature was $362^{\circ} \mathrm{C}$.

The 300C 1 dpa X melt wire pack showed no signs of melting indicating the melt wire pack never reached a temperature of $238.6^{\circ} \mathrm{C}$. According to the model, if the melt wire pack is at $258^{\circ} \mathrm{C}$ then the average specimen temperature is $298^{\circ} \mathrm{C}$ (information from ECAR-3575, Table 11). This means the actual temperature based on the melt wires is below $279^{\circ} \mathrm{C}$. Comparing this to the calculated as-run temperatures, the average specimen temperature was $276^{\circ} \mathrm{C}$.

The 300C $1 \mathrm{dpa} Y$ melt wire pack showed that the experiments maximum melt wire temperature was between $238.6^{\circ} \mathrm{C}$ and $271.5^{\circ} \mathrm{C}$ which shows good agreement with Table 8-9. According to the model, if the melt wire pack is at $257^{\circ} \mathrm{C}$ then the average specimen temperature is $297^{\circ} \mathrm{C}$ (information from ECAR-3575, Table 11). This means the actual temperature based on the melt wires is somewhere between $279^{\circ} \mathrm{C}$ and $312^{\circ} \mathrm{C}$. Comparing this to the calculated as-run temperatures, the average specimen temperature was $272^{\circ} \mathrm{C}$.

The 400C $1 \mathrm{dpa} Y$ melt wire pack showed that the experiments maximum melt wire temperature was between $399.4^{\circ} \mathrm{C}$ and $449.5^{\circ} \mathrm{C}$. According to the model, if the melt wire pack is at $367^{\circ} \mathrm{C}$ then the average specimen temperature is $407^{\circ} \mathrm{C}$ (information from ECAR-3575, Table 11). This means the actual temperature based on the melt wires is somewhere between $439^{\circ} \mathrm{C}$ and $490^{\circ} \mathrm{C}$. Comparing this to the calculated as-run temperatures, the average specimen temperature was $371^{\circ} \mathrm{C}$.

\section{ACRONYMS}

$\begin{array}{ll}\text { ALWR } & \text { Advance Light Water Reactor } \\ \text { ATR } & \text { Advanced Test Reactor } \\ \text { BSU } & \text { Boise State University } \\ \text { CT } & \text { Compact Tension } \\ \text { CUI } & \text { Controlled Unclassified Information } \\ \text { EA } & \text { Enterprise Architecture } \\ \text { ECAR } & \text { Engineering Calculations and Analysis Report } \\ \text { EJ } & \text { Engineering Job } \\ \text { INL } & \text { Idaho National Laboratory } \\ \text { LWP } & \text { Laboratory Wide Procedure } \\ \text { LWR } & \text { Light Water Reactor } \\ \text { NSH } & \text { Neck Shim Housing } \\ \text { NSUF } & \text { Nuclear Science user Facilities } \\ \text { PM-HIP } & \text { Powder Metallurgy and Hot Isostatic Pressing }\end{array}$

\footnotetext{
${ }^{1}$ The average specimen temperature was calculated by averaging each specimen individually except for the TEM's. The TEM's were averaged for each position and then used in the overall average.
} 
SAR

SMR

SSC

SST

TEM

TFR

TRA
Safety Analysis Report

Small Modular Reactor

System, Structure, or Component

Stainless Steel

Transmission Electron Microscope

Technical and Functional Requirements

Test Reactor Area 


\section{DATA FILES}

The following table contains names and descriptions of the electronic files associated with this analysis that can be found in the following directories:

\begin{tabular}{|c|c|}
\hline \multicolumn{2}{|c|}{ IIfswcb1|projectsIUSUFIIRRADIATION TESTINGINSUFIBSU-8242\As-Run } \\
\hline Filename & Description \\
\hline ECAR-5510.docx & This document \\
\hline Appendix B - BSU-8242 AR HRs.xmcd & $\begin{array}{l}\text { Mathcad file containing material properties and heat } \\
\text { rates }\end{array}$ \\
\hline \multicolumn{2}{|c|}{ IIfswcb1|projectsIUSUFIIRRADIATION TESTINGINSUFIBSU-8242VAs-RunIAbaqus|300C_3dpa } \\
\hline Filename & Description \\
\hline BSU-8242_300C_3dpa_AsRun.cae & ABAQUS cae file \\
\hline BSU-8242_300C_3dpa “.inp, .odb” & $\begin{array}{l}\text { ABAQUS input and output files for the 300C 3dpa } \\
\text { capsule }\end{array}$ \\
\hline \multicolumn{2}{|c|}{ IIfswcb1|projectsIUSUFIIRRADIATION TESTINGINSUFIBSU-8242VAs-Run\Abaqus|400C_3dpa_X } \\
\hline Filename & Description \\
\hline BSU-8242_400C_3dpa_X_AsRun.cae & ABAQUS cae file \\
\hline BSU-8242_400C_3dpa_X“.inp, .odb” & $\begin{array}{l}\text { ABAQUS input and output files for the } 400 \mathrm{C} 3 \mathrm{dpa} X \\
\text { capsule }\end{array}$ \\
\hline \multicolumn{2}{|c|}{ IIfswcb1|projectsIUSUFIIRRADIATION TESTINGINSUFIBSU-8242\As-Run\Abaqus|400C_3dpa_Y } \\
\hline Filename & Description \\
\hline BSU-8242_400C_3dpa_Y_AsRun.cae & ABAQUS cae file \\
\hline BSU-8242_400C_3dpa_Y“.inp, .odb” & $\begin{array}{l}\text { ABAQUS input and output files for the } 400 \mathrm{C} 3 \mathrm{dpa} Y \\
\text { capsule }\end{array}$ \\
\hline \multicolumn{2}{|c|}{ IIfswcb1|projectsIUSUFIIRRADIATION TESTINGINSUFIBSU-8242VAs-Run\Abaqus|400C-300C_1dpa_X } \\
\hline Filename & Description \\
\hline BSU-8242_400C-300C_1dpa_X.cae & ABAQUS cae file \\
\hline BSU-8242_400C-300C_1dpa_X“.inp, .odb” & $\begin{array}{l}\text { ABAQUS input and output files for the } 400 \mathrm{C}-300 \mathrm{C} 1 \mathrm{dpa} \\
\mathrm{X} \text { capsule }\end{array}$ \\
\hline \multicolumn{2}{|c|}{ IIfswcb1|projectsIUSUFIIRRADIATION TESTINGINSUFIBSU-8242VAs-RunIAbaqus\400C-300C_1dpa_Y } \\
\hline Filename & Description \\
\hline BSU-8242_400C-300C_1dpa_Y.cae & ABAQUS cae file \\
\hline BSU-8242_400C-300C_1dpa_Y“.inp, .odb” & $\begin{array}{l}\text { ABAQUS input and output files for the } 400 \mathrm{C}-300 \mathrm{C} 1 \mathrm{dpa} \\
\text { Y capsule }\end{array}$ \\
\hline
\end{tabular}




\section{CONCLUSIONS}

See cover page Section 11 for Conclusions

\section{REFERENCES}

[1] C. Hale, ECAR-3575, Rev. 3, "BSU-8242 Programmatic and Safety Compliance Structural and Thermal Analysis", 05/02/2018.

[2] J. Brookman, ECAR-4341, Rev. 1, "BSU-8242 1 DPA As-Run Physics Analysis", 5/28/2020.

[3] J. Brookman, ECAR-4951, Rev. 1, "BSU-8242 3 DPA As-Run Physics Analysis, 07/02/2020.

[4] K. L. Davis and L. A. Hone, INL EXT-20-58375, Rev. 0, "NSUF Melt Wire Evaluation for BSU-8242 and GE Hitachi-10393 Irradiation Experiments", April 2020.

[5] P. E. Murray, ECAR-131, Rev 0, "Validation of ABAQUS Standard 6.7-3 Heat Transfer", 1/30/2008.

[6] ABAQUS Standard, Version 6.14-2, Dassault Systemes Corp., Inc., Providence, RI, 2014.

[7] Idaho National Laboratory, LWP-10200, Rev 11, "Engineering Calculations and Analysis Report", 02/06/2018.

[8] Idaho National Laboratory, LWP-10200, Rev 9, "Engineering Calculations and Analysis Report", $5 / 17 / 2017$.

\section{APPENDICES}

Appendix A - Engineering Inputs

Appendix B - Heat Rates

Appendix C - Computer Code Validation 
Appendix A

\section{Engineering Inputs}


Perform an as-run thermal analysis of the BSU-8242 experiment using physics results from ECAR-4341 and ECAR-4951. Temperature reported for each specimen shall be the specimen average and specimen maximum using the cycle average heating rates. 
Appendix B

Heat Rates 


\section{B.1 Material Densities:}

In order to convert specimen heat rates into values used in ABAQUS, the densities for each specimen is needed. The densites for each specime are obtained from ECAR-3575, Appendix C.

$$
\begin{gathered}
\rho_{625}:=8.44 \frac{\mathrm{gm}}{\mathrm{cm}^{3}} \\
\rho_{690}:=8.19 \frac{\mathrm{gm}}{\mathrm{cm}^{3}} \\
\rho_{\mathrm{G} 91}:=7.805 \frac{\mathrm{gm}}{\mathrm{cm}^{3}} \\
\rho_{304 \mathrm{~L}}:=8.03 \frac{\mathrm{gm}}{\mathrm{cm}^{3}} \\
\rho_{316 \mathrm{~L}}:=7.99 \frac{\mathrm{gm}^{3}}{\mathrm{~cm}^{3}} \\
\rho_{508}:=0.283 \frac{\mathrm{lb}^{3}}{\mathrm{in}^{3}} \\
\rho_{\mathrm{Al} 3 \mathrm{Hf} 20}:=3.428 \frac{\mathrm{gm}^{3}}{\mathrm{~cm}^{3}} \\
\rho_{\mathrm{Al} 3 \mathrm{Hf} 28}:=3.74 \frac{\mathrm{gm}^{3}}{\mathrm{~cm}^{3}} \\
:=3.95 \frac{\mathrm{gm}^{3}}{\mathrm{~cm}^{3}}
\end{gathered}
$$

\section{B.2 Heating Rates for Capsule 300C 1 DPA (X):}

Heating rates given in ECAR-4341 are given in specimen order starting from the top and going to the bottom. This same scheme will be kept here. During the neutronics modeling of this capsule the TEM's in position 2 (the USU specimens) were flipped so that the Al3Hf2O's were on top instead of the Al3Hf36's. Since the TEM's are so small the heating rate difference would be negligible if the model was corrected and reran. Instead, the heat rates from the neutronics analysis will be flipped so the heat rates associated for the right material type is used. 


\section{BSU-8242 As-Run Thermal Analysis}

HR $_{\text {Splits }}:=\left(\begin{array}{cccc}6.27 & 6.38 & 6.56 & 6.53 \\ 5.8 & 5.95 & 6.12 & 6.12 \\ 5.68 & 5.81 & 6.01 & 5.99 \\ 8.82 & 10.08 & 11.06 & 11.48 \\ 9.83 & 10.23 & 11.2 & 11.6 \\ 9.71 & 10.24 & 11.29 & 11.49 \\ 9.01 & 9.33 & 10.13 & 10.71 \\ 9.03 & 9.49 & 10.04 & 10.35 \\ 9.07 & 9.44 & 10.01 & 10.18 \\ 8.06 & 8.13 & 9 & 9.17 \\ 8.11 & 8.11 & 8.9 & 9.18 \\ 7.99 & 8.43 & 8.79 & 9.1 \\ 5.07 & 5.35 & 5.57 & 5.58 \\ 4.88 & 5.17 & 5.32 & 5.35 \\ 5.04 & 5.19 & 5.33 & 5.42 \\ 5.14 & 5.37 & 5.38 & 5.43 \\ 5.08 & 5.17 & 5.33 & 5.45 \\ 5.01 & 5.09 & 5.17 & 5.36 \\ 5.02 & 5.16 & 5.32 & 5.32 \\ 5.01 & 5.1 & 5.36 & 5.48 \\ 5.04 & 5.17 & 5.24 & 5.45 \\ 5.11 & 5.12 & 5.4 & 5.53 \\ 5.14 & 5.15 & 5.24 & 5.5 \\ 4.96 & 5.11 & 5.34 & 5.39 \\ 4.79 & 4.98 & 5.15 & 5.16 \\ 3.87 & 4.02 & 4.23 & 4.26\end{array}\right) \quad\left(\begin{array}{c}127 \\ 126 \\ 123 \\ 122 \\ 121 \\ 120 \\ 119 \\ 118 \\ 117 \\ 116 \\ 115 \\ 114 \\ 113 \\ 112 \\ 111 \\ 110 \\ 109 \\ 108 \\ 107 \\ 106 \\ 105 \\ 104 \\ 103 \\ 102 \\ 101\end{array}\right)$

$i:=0$.. length $\left(\mathrm{HR}_{\text {Splits }}\langle 0\rangle\right)-1$

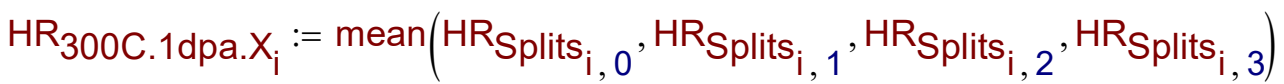




$\mathrm{HR}_{300 \mathrm{C} .1 \mathrm{dpa} . \mathrm{X}}=\left(\begin{array}{c}6.435 \\ 5.997 \\ 5.872 \\ 10.36 \\ 10.715 \\ 10.682 \\ 9.795 \\ 9.727 \\ 9.675 \\ 8.59 \\ 8.575 \\ 8.578 \\ 5.393 \\ 5.185 \\ 5.202 \\ 5.225 \\ 5.33 \\ 5.258 \\ 5.157 \\ 5.205 \\ 5.237 \\ 5.225 \\ 5.29 \\ 5.258 \\ 5.2 \\ 5.02 \\ 4.095\end{array}\right) \cdot \frac{\mathrm{W}}{\mathrm{gm}}\left(\begin{array}{c}127 \\ 126 \\ 125 \\ 124 \\ 123 \\ 122 \\ 121 \\ 120 \\ 119 \\ 118 \\ 117 \\ 116 \\ 115 \\ 114 \\ 113 \\ 112 \\ 111 \\ 110 \\ 109 \\ 108 \\ 107 \\ 106 \\ 105 \\ 104 \\ 103 \\ 102 \\ 101\end{array}\right)$




$\rho_{300 \mathrm{C} .1 \mathrm{dpa} . \mathrm{X}}:=\left(\begin{array}{c}\rho_{508} \\ \rho_{508} \\ \rho_{508} \\ \rho_{\mathrm{Al} 3 \mathrm{Hf} 36} \\ \rho_{\mathrm{Al} 3 \mathrm{Hf} 36} \\ \rho_{\mathrm{Al} 3 \mathrm{Hf} 36} \\ \rho_{\mathrm{Al} 3 \mathrm{Hf} 28} \\ \rho_{\mathrm{Al} 3 \mathrm{Hf} 28} \\ \rho_{\mathrm{Al} 3 \mathrm{Hf} 28} \\ \rho_{\mathrm{Al} 3 \mathrm{Hf} 20} \\ \rho_{\mathrm{Al} 3 \mathrm{Hf} 20} \\ \rho_{\mathrm{Al} 3 \mathrm{Hf} 20} \\ \rho_{508} \\ \rho_{508} \\ \rho_{508} \\ \rho_{508} \\ \rho_{508} \\ \rho_{508} \\ \rho_{508} \\ \rho_{508} \\ \rho_{508} \\ \rho_{508} \\ \rho_{508} \\ \rho_{508} \\ \end{array}\right)$

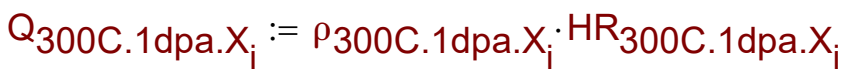




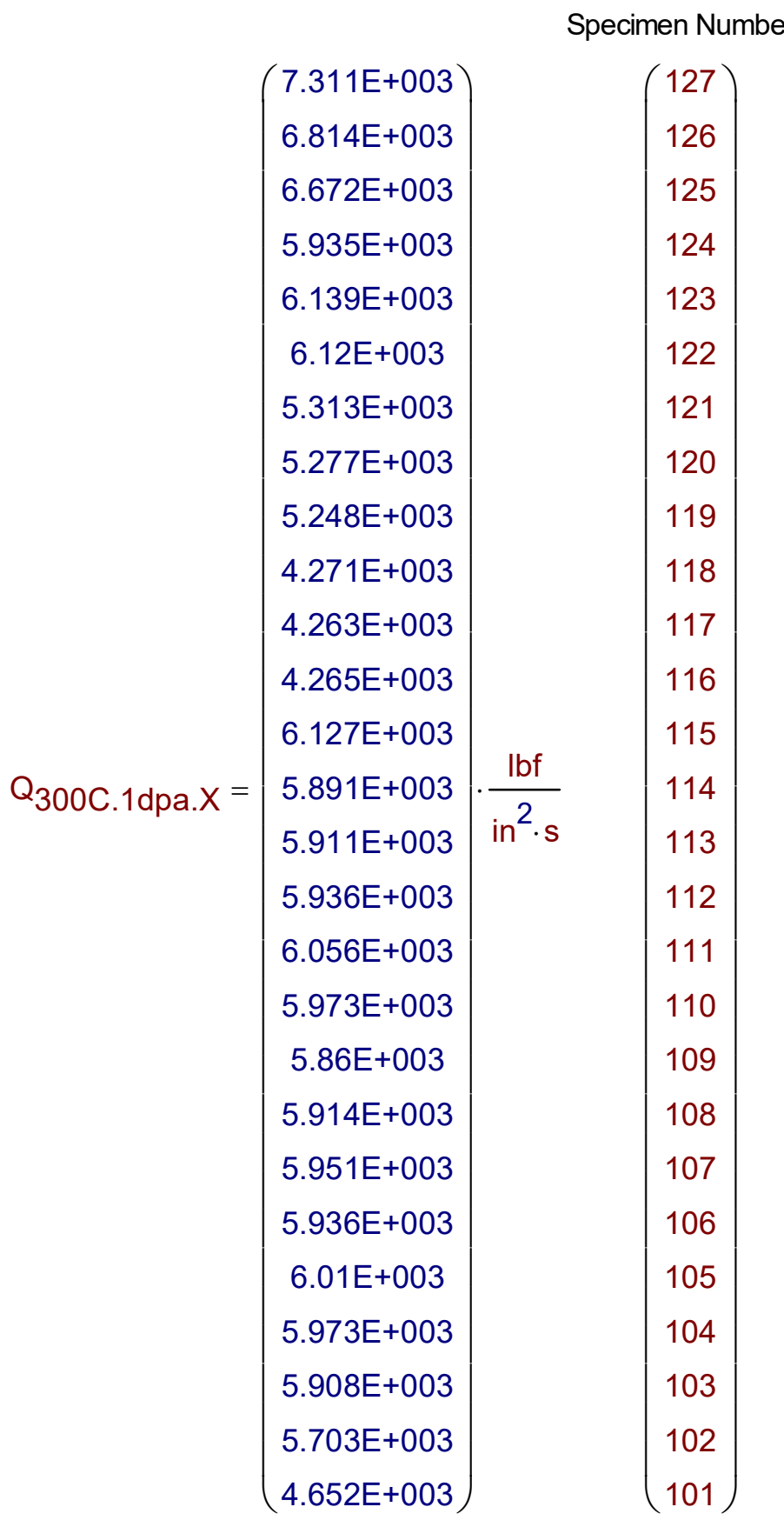




\section{B.3 Heating Rates for Capsule 300C 1 DPA (Y):}

Specimen Number

Hinsplitsn $:=\left(\begin{array}{cccc}6.17 & 6.28 & 6.42 & 6.39 \\ 5.78 & 5.94 & 6.16 & 6.14 \\ 5.65 & 5.78 & 6.01 & 5.98 \\ 4.94 & 5.09 & 5.3 & 5.33 \\ 4.79 & 4.97 & 5.17 & 5.17 \\ 3.82 & 3.98 & 4.17 & 4.18\end{array}\right) \frac{\mathrm{g}}{\mathrm{gm}}\left(\begin{array}{c}206 \\ 205 \\ 204 \\ 203 \\ 202 \\ 201\end{array}\right)$

$\mathrm{i}:=0$.. length $\left(\mathrm{HR}_{\text {Splits }}\langle 0\rangle\right)-1$

HR $_{300 \text { C.1dpa.Y }}:=$ mean $_{\mathrm{i}}$ HR $_{\text {Splits }_{i, 0}}$, HR $_{\text {Splits }_{i, 1}}$, HR $_{\text {Splits }_{i, 2}}$, HR $\left._{\text {Splits }_{i, 3}}\right)$

Specimen Number

$\mathrm{HR}_{300 \mathrm{C} .1 \mathrm{dpa} . Y}=\left(\begin{array}{l}6.315 \\ 6.005 \\ 5.855 \\ 5.165 \\ 5.025 \\ 4.037\end{array}\right) \cdot \frac{\mathrm{W}}{\mathrm{gm}} \quad\left(\begin{array}{c}206 \\ 205 \\ 204 \\ 203 \\ 202 \\ 201\end{array}\right)$

$\rho_{300 \text { C.1dpa. }}:=\left(\begin{array}{c}\rho_{508} \\ \rho_{316 L} \\ \rho_{316 L} \\ \rho_{316 L} \\ \rho_{316 L} \\ \rho_{508}\end{array}\right)$

$Q_{300 C .1 d p a . Y_{i}}:=\rho_{300 C .1 d p a . Y_{i}} \cdot H_{300 C .1 d p a . Y_{i}}$ 
Specimen Number

$Q_{300 \text { C.1dpa. } Y}=\left(\begin{array}{l}7.175 \mathrm{E}+003 \\ 6.959 \mathrm{E}+003 \\ 6.785 \mathrm{E}+003 \\ 5.985 \mathrm{E}+003 \\ 5.823 \mathrm{E}+003 \\ 4.587 \mathrm{E}+003\end{array}\right) \cdot \frac{\mathrm{lbf}}{\mathrm{in}^{2} \cdot \mathrm{s}} \cdot\left(\begin{array}{c}206 \\ 205 \\ 204 \\ 203 \\ 202 \\ 201\end{array}\right)$

\section{B.4 Heating Rates for Capsule 300C 3 DPA:}

During the neutronics modeling of this capsule the TEM's in position 2 (the USU specimens) were flipped so that the Al3Hf36's were on top instead of the Al3Hf20's. Since the TEM's are so small the heating rate difference would be negligible if the model was corrected and reran. Instead, the heat rates from the neutronics analysis will be flipped so the heat rates associated for the right material type is used. 


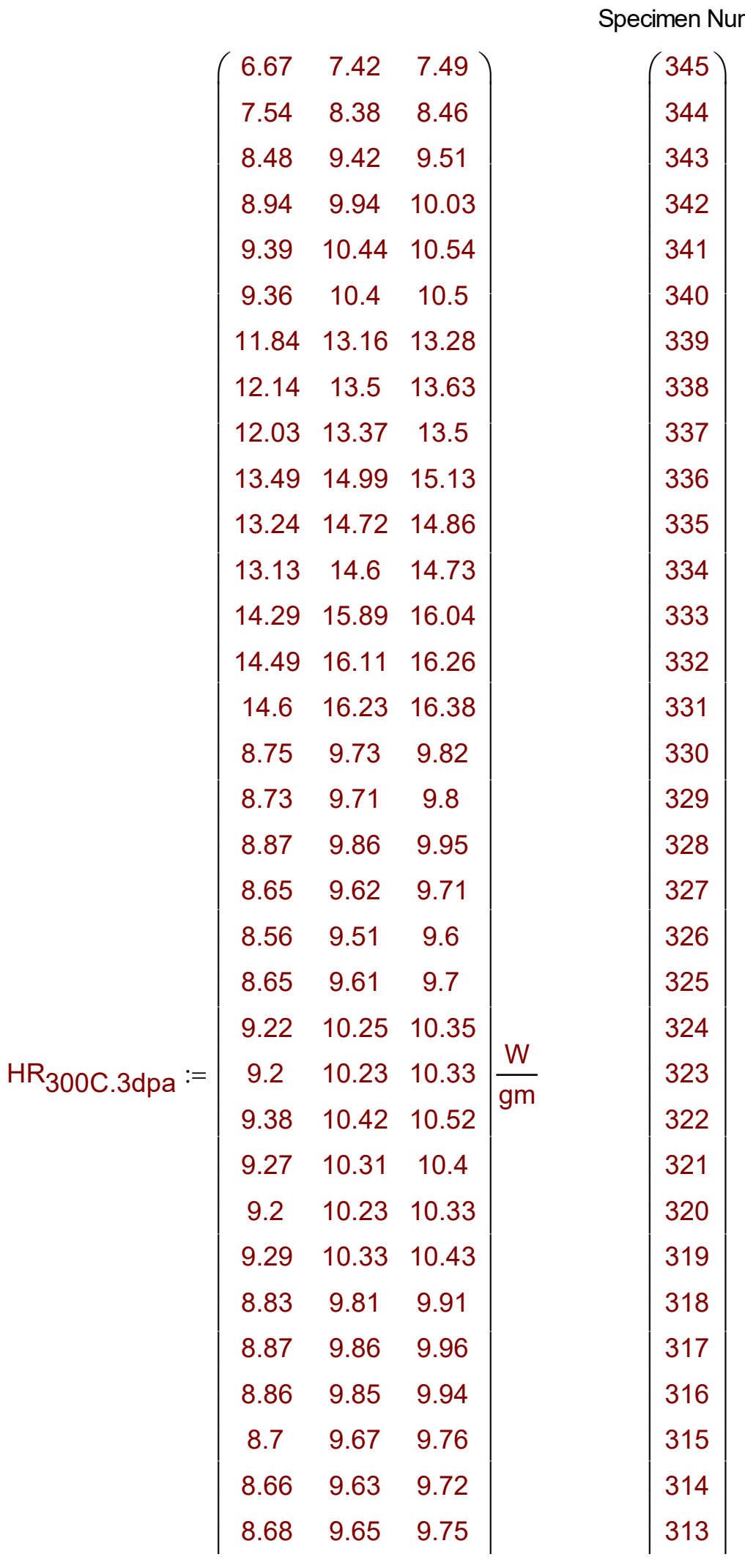




$\left.\left.\mid \begin{array}{ccc}9.44 & 10.5 & 10.6 \\ 9.38 & 10.43 & 10.53 \\ 9.3 & 10.34 & 10.44 \\ 9.24 & 10.27 & 10.37 \\ 9.3 & 10.34 & 10.44 \\ 9.33 & 10.37 & 10.47 \\ 9.4 & 10.45 & 10.55 \\ 9.38 & 10.43 & 10.52 \\ 9.04 & 10.05 & 10.15 \\ 8.72 & 9.69 & 9.79 \\ 7.9 & 8.78 & 8.86 \\ 7.17 & 7.97 & 8.05\end{array}\right) \quad \mid \begin{array}{l}312 \\ 311 \\ 310 \\ 309 \\ 308 \\ 307 \\ 306 \\ 305 \\ 304 \\ 303 \\ 302 \\ 301\end{array}\right)$

$$
\left(\begin{array}{c}
\rho_{690} \\
\rho_{690} \\
\rho_{625} \\
\rho_{625} \\
\rho_{625} \\
\rho_{625} \\
\rho_{\mathrm{Al} 3 \mathrm{Hf} 20} \\
\rho_{\mathrm{Al} 3 \mathrm{Hf} 20} \\
\rho_{\mathrm{Al} 3 \mathrm{Hf} 20} \\
\rho_{\mathrm{Al} 3 \mathrm{Hf} 28} \\
\rho_{\mathrm{Al} 3 \mathrm{Hf} 28} \\
\rho_{\mathrm{Al} 3 \mathrm{Hf} 28} \\
\rho_{\mathrm{Al} 3 \mathrm{Hf} 36} \\
\rho_{\mathrm{Al} 3 \mathrm{Hf} 36} \\
\rho_{\mathrm{Al} 3 \mathrm{Hf} 36} \\
\rho_{690}
\end{array}\right.
$$




\begin{tabular}{|c|c|}
\hline & $\rho_{690}$ \\
\hline & $\rho_{690}$ \\
\hline & $\rho_{690}$ \\
\hline & $\rho_{690}$ \\
\hline & $\rho_{690}$ \\
\hline & $\rho 625$ \\
\hline$\rho_{300 C .3 d p a}:=$ & $\rho 625$ \\
\hline & $\rho_{625}$ \\
\hline & $\rho_{625}$ \\
\hline & $\rho_{625}$ \\
\hline & $\rho 625$ \\
\hline & $\rho 690$ \\
\hline & $\rho 690$ \\
\hline & $\rho 690$ \\
\hline & $\rho 690$ \\
\hline & $\rho_{690}$ \\
\hline & $\rho_{690}$ \\
\hline & $\rho 625$ \\
\hline & $\rho_{625}$ \\
\hline & $\rho_{625}$ \\
\hline & $\rho_{625}$ \\
\hline & $\rho_{625}$ \\
\hline & $\rho_{625}$ \\
\hline & $\rho_{625}$ \\
\hline & $\rho_{625}$ \\
\hline & $\rho_{625}$ \\
\hline & $\rho 625$ \\
\hline & $\rho_{690}$ \\
\hline & \\
\hline
\end{tabular}


$Q_{300 C .3 d p a_{i, j}}:=\rho_{300 C .3 d p a_{i}} \cdot H_{300 C .3 d p a}, j$

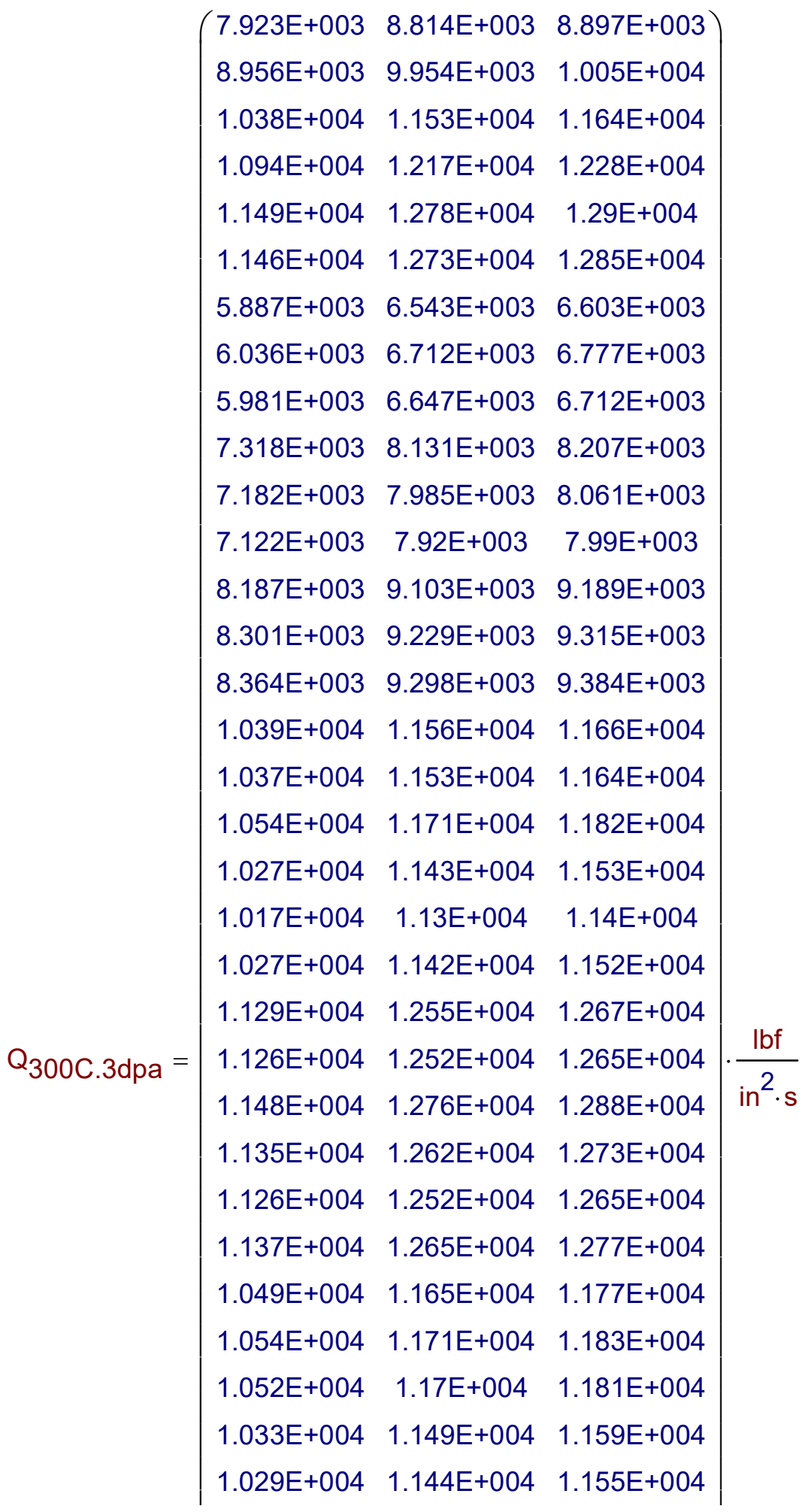

Specimen Number

$\begin{aligned} & 345 \\ & 344 \\ & 343 \\ & 342 \\ & 341 \\ & 340 \\ & 339 \\ & 338 \\ & 337 \\ & 336 \\ & 335 \\ & 334 \\ & 333 \\ & 332 \\ & 331 \\ & 330 \\ & 329 \\ & 328 \\ & 327 \\ & 326 \\ & 325 \\ & 324 \\ & 323 \\ & 322 \\ & 321 \\ & 320 \\ & 319 \\ & 318 \\ & 317 \\ & 316 \\ & 315 \\ & 314\end{aligned} \mid$


BSU-8242 As-Run Thermal Analysis

$\left.\left.\begin{array}{|ccc}1.031 E+004 & 1.146 E+004 & 1.158 E+004 \\ 1.156 E+004 & 1.285 E+004 & 1.298 E+004 \\ 1.148 E+004 & 1.277 E+004 & 1.289 E+004 \\ 1.138 E+004 & 1.266 E+004 & 1.278 E+004 \\ 1.131 E+004 & 1.257 E+004 & 1.269 E+004 \\ 1.138 E+004 & 1.266 E+004 & 1.278 E+004 \\ 1.142 E+004 & 1.269 E+004 & 1.282 E+004 \\ 1.151 E+004 & 1.279 E+004 & 1.291 E+004 \\ 1.148 E+004 & 1.277 E+004 & 1.288 E+004 \\ 1.107 E+004 & 1.23 E+004 & 1.242 E+004 \\ 1.067 E+004 & 1.186 E+004 & 1.198 E+004 \\ 9.384 E+003 & 1.043 E+004 & 1.052 E+004 \\ 8.517 E+003 & 9.467 E+003 & 9.562 E+003\end{array}\right) \quad \mid \begin{array}{l}313 \\ 312 \\ 311 \\ 310 \\ 309 \\ 308 \\ 307 \\ 306 \\ 305 \\ 304 \\ 303 \\ 302 \\ 301\end{array}\right)$

\section{B.5 Heating Rates for Capsule 400C 1 DPA (X):}

Specimen Number

$\left(\begin{array}{cccc}3.71 & 3.8 & 4.09 & 4.11 \\ 5 & 5.11 & 5.42 & 5.44 \\ 5.89 & 5.95 & 6.23 & 6.24 \\ 6.87 & 6.89 & 7.12 & 7.11 \\ 7.91 & 7.91 & 8.12 & 8.09 \\ 7.98 & 7.98 & 8.18 & 8.14 \\ 8.31 & 8.22 & 8.23 & 8.26 \\ 8.05 & 8.1 & 8.1 & 8.23 \\ 8.11 & 8 & 8.19 & 8.34 \\ 8.04 & 7.88 & 8.28 & 8.29 \\ 8.09 & 7.89 & 8.16 & 8.09 \\ 8.1 & 7.95 & 8.2 & 8.26 \\ 7.93 & 7.96 & 8.09 & 8.23 \\ 8.08 & 7.96 & 8.19 & 8.24 \\ 8.26 & 7.87 & 8.23 & 8.35 \\ 8.15 & 7.97 & 8.21 & 8.28 \\ 8.04 & 7.83 & 8.28 & 8.27 \\ 8.12 & 7.9 & 8.24 & 8.2\end{array}\right) \quad\left(\begin{array}{l}460 \\ 459 \\ 458 \\ 457 \\ 456 \\ 455 \\ 454 \\ 453 \\ 452 \\ 451 \\ 450 \\ 449 \\ 448 \\ 447 \\ 446 \\ 445 \\ 444 \\ 443\end{array}\right)$




\section{BSU-8242 As-Run Thermal Analysis}

\begin{tabular}{|c|c|c|c|c|c|c|}
\hline & 7.44 & 7.33 & 7.35 & 7.25 & & 442 \\
\hline & 7.32 & 7.12 & 7.45 & 7.42 & & 441 \\
\hline & 7.36 & 7.17 & 7.48 & 7.41 & & 440 \\
\hline & 7.5 & 7.26 & 7.42 & 7.49 & & 439 \\
\hline & 7.43 & 7.39 & 7.45 & 7.31 & & 438 \\
\hline & 7.47 & 7.24 & 7.4 & 7.31 & & 437 \\
\hline & 7.54 & 7.46 & 7.54 & 7.65 & & 436 \\
\hline & 7.54 & 7.54 & 7.58 & 7.58 & & 435 \\
\hline & 7.66 & 7.48 & 7.72 & 7.49 & & 434 \\
\hline & 7.58 & 7.5 & 7.62 & 7.48 & & 433 \\
\hline & 7.84 & 7.51 & 7.56 & 7.5 & & 432 \\
\hline & 7.57 & 7.56 & 7.64 & 7.52 & W & 431 \\
\hline spplitsin:- & 7.48 & 7.5 & 7.55 & 7.5 & $\overline{\mathrm{gm}}$ & 430 \\
\hline & 7.46 & 7.53 & 7.49 & 7.34 & & 429 \\
\hline & 7.5 & 7.59 & 7.44 & 7.34 & & 428 \\
\hline & 7.65 & 7.53 & 7.49 & 7.37 & & 427 \\
\hline & 7.68 & 7.53 & 7.71 & 7.59 & & 426 \\
\hline & 7.55 & 7.46 & 7.78 & 7.66 & & 425 \\
\hline & 7.5 & 7.52 & 7.76 & 7.57 & & 424 \\
\hline & 7.68 & 7.4 & 7.66 & 7.51 & & 423 \\
\hline & 7.62 & 7.46 & 7.62 & 7.58 & & 422 \\
\hline & 7.62 & 7.66 & 7.52 & 7.41 & & 421 \\
\hline & 7.49 & 7.56 & 7.53 & 7.35 & & 420 \\
\hline & 7.64 & 7.63 & 7.71 & 7.56 & & 419 \\
\hline & 7.67 & 7.88 & 8.18 & 7.96 & & 418 \\
\hline & 7.58 & 7.87 & 7.91 & 7.77 & & 417 \\
\hline & 7.76 & 7.83 & 7.83 & 7.81 & & 416 \\
\hline & 7.8 & 7.82 & 7.95 & 7.9 & & 415 \\
\hline & 7.75 & 7.96 & 7.95 & 7.85 & & 414 \\
\hline & 7.78 & 7.89 & 7.92 & 7.83 & & 413 \\
\hline & 7.93 & 7.98 & 8.14 & 8.03 & & 412 \\
\hline & 8.05 & 7.93 & 8.37 & 8.19 & & 411 \\
\hline & 7.86 & 7.77 & 8.28 & 8.12 & & 410 \\
\hline & 7.86 & 7.75 & 8.18 & 7.92 & & 409 \\
\hline & 7.9 & 7.78 & 8.07 & 7.78 & & 408 \\
\hline & 7.89 & 7.85 & 8.04 & 7.91 & & 407 \\
\hline
\end{tabular}




\section{BSU-8242 As-Run Thermal Analysis}

$\left.\left(\begin{array}{cccc}8.03 & 7.98 & 8.08 & 8.05 \\ 8.09 & 8.07 & 8.15 & 8.12 \\ 7.81 & 7.75 & 7.78 & 7.72 \\ 7.93 & 7.87 & 7.87 & 7.8 \\ 7.85 & 7.8 & 7.81 & 7.76 \\ 7.56 & 7.57 & 7.6 & 7.55\end{array}\right) \quad \mid \begin{array}{l}406 \\ 405 \\ 404 \\ 403 \\ 402 \\ 401\end{array}\right)$

$i:=0 .$. length $\left(\mathrm{HR}_{\text {Splits }}\langle 0\rangle\right)-1$

HR $_{400 C .1 d p a . X_{i}}:=$ mean $\left(\right.$ HR $_{\text {Splits }_{i, 0}}$, HR $_{\text {Splits }_{i, 1}}$, HR $_{\text {Splits }_{i, 2}}$, HR $\left._{\text {Splits }_{i, 3}}\right)$

\section{Specimen Number}

$\left(\begin{array}{c}3.928 \\ 5.242 \\ 6.077 \\ 6.997 \\ 8.008 \\ 8.07 \\ 8.255 \\ 8.12 \\ 8.16 \\ 8.123 \\ 8.057 \\ 8.127 \\ 8.053 \\ 8.117 \\ 8.178 \\ 8.152 \\ 8.105 \\ 8.115 \\ 7.343 \\ 7.327 \\ 7.355 \\ 7.418 \\ 7.395 \\ 7.355\end{array}\right) \quad\left(\begin{array}{l}460 \\ 459 \\ 458 \\ 457 \\ 456 \\ 455 \\ 454 \\ 453 \\ 452 \\ 451 \\ 450 \\ 449 \\ 448 \\ 447 \\ 446 \\ 445 \\ 444 \\ 443 \\ 442 \\ 441 \\ 440 \\ 439 \\ 438 \\ 437\end{array}\right)$




\begin{tabular}{|c|c|c|c|}
\hline \multirow{36}{*}{$\mathrm{HR}_{400 \mathrm{C} .1 \mathrm{dpa} . \mathrm{X}=}$} & 7.548 & & 436 \\
\hline & 7.56 & & 435 \\
\hline & 7.588 & & 434 \\
\hline & 7.545 & & 433 \\
\hline & 7.603 & & 432 \\
\hline & 7.572 & W & 431 \\
\hline & 7.508 & $\overline{\mathrm{gm}}$ & 430 \\
\hline & 7.455 & & 429 \\
\hline & 7.468 & & 428 \\
\hline & 7.51 & & 427 \\
\hline & 7.628 & & 426 \\
\hline & 7.612 & & 425 \\
\hline & 7.588 & & 424 \\
\hline & 7.563 & & 423 \\
\hline & 7.57 & & 422 \\
\hline & 7.553 & & 421 \\
\hline & 7.482 & & 420 \\
\hline & 7.635 & & 419 \\
\hline & 7.923 & & 418 \\
\hline & 7.782 & & 417 \\
\hline & 7.808 & & 416 \\
\hline & 7.867 & & 415 \\
\hline & 7.878 & & 414 \\
\hline & 7.855 & & 413 \\
\hline & 8.02 & & 412 \\
\hline & 8.135 & & 411 \\
\hline & 8.008 & & 410 \\
\hline & 7.928 & & 409 \\
\hline & 7.883 & & 408 \\
\hline & 7.923 & & 407 \\
\hline & 8.035 & & 406 \\
\hline & 8.107 & & 405 \\
\hline & 7.765 & & 404 \\
\hline & 7.867 & & 403 \\
\hline & 7.805 & & 402 \\
\hline & 7.57 & & 401 \\
\hline
\end{tabular}




$\left(\begin{array}{l}\rho_{625} \\ \rho_{625} \\ \rho_{690} \\ \rho_{690} \\ \rho_{625} \\ \rho_{625} \\ \rho_{625} \\ \rho_{625} \\ \rho_{625} \\ \rho_{625} \\ \rho_{625} \\ \rho_{625} \\ \rho_{625} \\ \rho_{625} \\ \rho_{625} \\ \rho_{625} \\ \rho_{625} \\ \rho_{625} \\ \rho_{508} \\ \rho_{508} \\ \rho_{508} \\ \rho_{508} \\ \rho_{508} \\ \rho_{508} \\ \rho_{508} \\ \end{array}\right.$




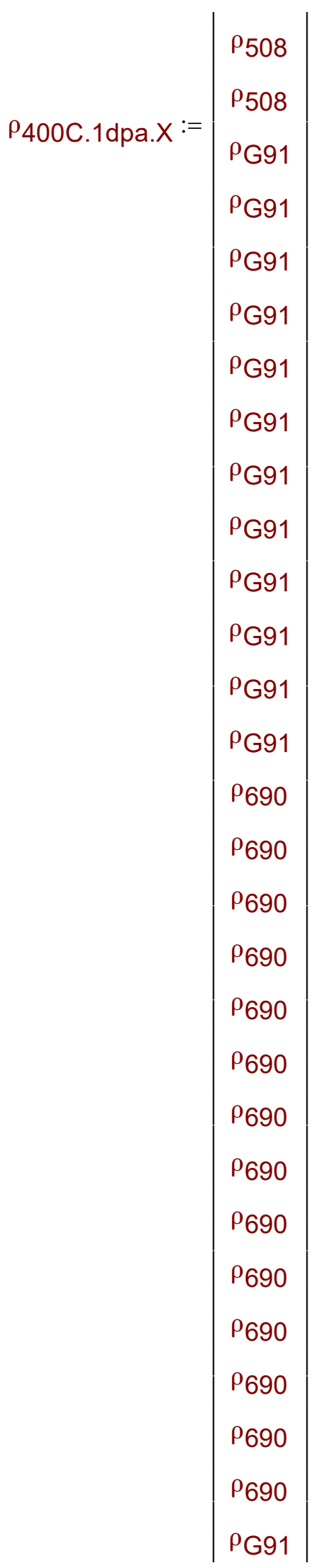




$$
\left(\begin{array}{c}
\rho_{G 91} \\
\rho_{508} \\
\rho_{508}
\end{array}\right)
$$

$Q_{400 C .1 d p a . X_{i}}:=\rho_{400 C .1 d p a . X_{i}} \cdot H^{400 C .1 d p a . X_{i}}$ Specimen Number

$\left(\begin{array}{c}4.808 \mathrm{E}+003 \\ 6.417 \mathrm{E}+003 \\ 7.219 \mathrm{E}+003 \\ 8.312 \mathrm{E}+003 \\ 9.802 \mathrm{E}+003 \\ 9.879 \mathrm{E}+003 \\ 1.011 \mathrm{E}+004 \\ 9.94 \mathrm{E}+003 \\ 9.989 \mathrm{E}+003 \\ 9.943 \mathrm{E}+003 \\ 9.863 \mathrm{E}+003 \\ 9.949 \mathrm{E}+003 \\ 9.857 \mathrm{E}+003 \\ 9.937 \mathrm{E}+003 \\ 1.001 \mathrm{E}+004 \\ 9.98 \mathrm{E}+003 \\ 9.921 \mathrm{E}+003 \\ 9.934 \mathrm{E}+003 \\ 8.342 \mathrm{E}+003 \\ 8.325 \mathrm{E}+003 \\ 8.356 \mathrm{E}+003 \\ 8.427 \mathrm{E}+003 \\ 8.402 \mathrm{E}+003 \\ 8.356 \mathrm{E}+003 \\ 8.575 \mathrm{E}+003 \\ 8.589 \mathrm{E}+003 \\ 8.62 \mathrm{E}+003 \\ 8.572 \mathrm{E}+003 \\ 8.638 \mathrm{E}+003\end{array}\right) \quad\left(\begin{array}{l}460 \\ 459 \\ 458 \\ 457 \\ 456 \\ 455 \\ 454 \\ 453 \\ 452 \\ 451 \\ 450 \\ 449 \\ 448 \\ 447 \\ 446 \\ 445 \\ 444 \\ 443 \\ 442 \\ 441 \\ 440 \\ 439 \\ 438 \\ 437 \\ 436 \\ 435 \\ 4 \\ 9\end{array}\right)$




\begin{tabular}{|c|c|c|c|}
\hline \multirow{31}{*}{$Q_{400 \text { C.1dpa.X }}=$} & $8.603 E+003$ & lbf & 431 \\
\hline & $8.499 E+003$ & $i^{2} \cdot s$ & 430 \\
\hline & $8.439 E+003$ & & 429 \\
\hline & $8.453 \mathrm{E}+003$ & & 428 \\
\hline & $8.501 \mathrm{E}+003$ & & 427 \\
\hline & $8.634 \mathrm{E}+003$ & & 426 \\
\hline & $8.617 E+003$ & & 425 \\
\hline & $8.589 E+003$ & & 424 \\
\hline & $8.561 \mathrm{E}+003$ & & 423 \\
\hline & $8.569 E+003$ & & 422 \\
\hline & $8.55 E+003$ & & 421 \\
\hline & 8.47E+003 & & 420 \\
\hline & $8.643 E+003$ & & 419 \\
\hline & $9.411 E+003$ & & 418 \\
\hline & $9.245 E+003$ & & 417 \\
\hline & $9.274 \mathrm{E}+003$ & & 416 \\
\hline & $9.345 E+003$ & & 415 \\
\hline & $9.357 E+003$ & & 414 \\
\hline & $9.331 E+003$ & & 413 \\
\hline & $9.527 \mathrm{E}+003$ & & 412 \\
\hline & $9.663 E+003$ & & 411 \\
\hline & $9.512 E+003$ & & 410 \\
\hline & $9.417 E+003$ & & 409 \\
\hline & $9.363 \mathrm{E}+003$ & & 408 \\
\hline & $9.411 E+003$ & & 407 \\
\hline & $9.544 \mathrm{E}+003$ & & 406 \\
\hline & $9.631 \mathrm{E}+003$ & & 405 \\
\hline & $8.79 E+003$ & & 404 \\
\hline & $8.906 E+003$ & & 403 \\
\hline & $8.868 E+003$ & & 402 \\
\hline & $8.601 \mathrm{E}+003$ & & 401 \\
\hline
\end{tabular}




\section{B.6 Heating Rates for Capsule 400C 1 DPA (Y):}

Hirsplitsn: $\left.=\left(\begin{array}{cccc}3.67 & 3.76 & 4.03 & 4.06 \\ 4.93 & 5.02 & 5.33 & 5.36 \\ 5.78 & 5.86 & 6.12 & 6.12 \\ 6.77 & 6.79 & 7.00 & 6.99 \\ 7.41 & 7.41 & 7.58 & 7.53 \\ 7.44 & 7.47 & 7.63 & 7.60 \\ 10.74 & 10.70 & 11.26 & 11.59 \\ 10.70 & 10.90 & 11.04 & 11.59 \\ 10.75 & 10.79 & 11.10 & 11.65 \\ 12.20 & 12.47 & 12.51 & 12.92 \\ 12.18 & 12.26 & 12.60 & 12.93 \\ 13.51 & 13.47 & 12.60 & 13.05 \\ 13.31 & 13.19 & 13.80 & 14.20 \\ 13.18 & 13.14 & 13.92 & 14.27 \\ 7.56 & 7.56 & 7.60 & 7.55 \\ 7.65 & 7.62 & 7.64 & 7.58 \\ 7.66 & 7.62 & 7.61 & 7.56 \\ 7.76 & 7.72 & 7.71 & 7.65 \\ 7.69 & 7.67 & 7.66 & 7.60 \\ 7.42 & 7.44 & 7.47 & 7.44\end{array}\right) \quad \begin{array}{l}521 \\ 519 \\ 518 \\ 516 \\ 515 \\ 514 \\ 513 \\ 512 \\ 511 \\ 510 \\ 509 \\ 508 \\ 507 \\ 506 \\ 505 \\ 504 \\ 503 \\ 502 \\ 501\end{array}\right)$

$\mathrm{i}:=0$.. length $\left(\mathrm{HR}_{\text {Splits }}\langle 0\rangle\right)-1$

HR $_{400 \text { C.1dpa.Y }}:=$ mean $_{\mathrm{i}}$ HR $_{\text {Splits }_{\mathrm{i}, 0}}$, HR $\left._{\text {Splits }_{\mathrm{i}, 1}}, \mathrm{HR}_{\text {Splits }_{\mathrm{i}, 2}}, \mathrm{HR}_{\text {Splits }_{\mathrm{i}, 3}}\right)$ 


$H_{400 C .1 d p a . Y}=\left(\begin{array}{c}3.88 \\ 5.16 \\ 5.97 \\ 6.888 \\ 7.482 \\ 7.535 \\ 11.072 \\ 11.057 \\ 11.072 \\ 12.525 \\ 12.508 \\ 12.575 \\ 13.785 \\ 13.563 \\ 13.627 \\ 7.567 \\ 7.622 \\ 7.612 \\ 7.71 \\ 7.655 \\ 7.442\end{array}\right) \cdot \frac{\mathrm{gm}}{520}\left(\begin{array}{l}521 \\ 519 \\ 518 \\ 517 \\ 516 \\ 515 \\ 514 \\ 511 \\ 512 \\ 509 \\ 508 \\ 507 \\ 506 \\ 505 \\ 504 \\ 503 \\ 502 \\ 501\end{array}\right)$




\begin{tabular}{|c|c|}
\hline & $\rho_{625}$ \\
\hline & $\rho_{625}$ \\
\hline & $\rho_{690}$ \\
\hline & $\rho_{690}$ \\
\hline & $\rho_{690}$ \\
\hline & $\rho_{690}$ \\
\hline & $\rho_{\mathrm{Al} 3 \mathrm{Hf} 20}$ \\
\hline & $\rho_{\mathrm{Al} 3 \mathrm{Hf} 20}$ \\
\hline & $\rho_{\mathrm{Al} 3 \mathrm{Hf} 20}$ \\
\hline & $\rho_{\mathrm{Al} 3 \mathrm{Hf} 28}$ \\
\hline$\rho_{400 \text { C.1dpa.Y }:=}$ & $\rho_{\mathrm{Al} 3 \mathrm{Hf} 28}$ \\
\hline & $\rho_{\mathrm{Al} 3 \mathrm{Hf} 28}$ \\
\hline & $\rho_{\mathrm{Al} 3 \mathrm{Hf} 36}$ \\
\hline & $\rho_{\mathrm{Al} 3 \mathrm{Hf} 36}$ \\
\hline & $\rho_{\mathrm{Al} 3 \mathrm{Hf} 36}$ \\
\hline & $\rho_{\mathrm{G} 91}$ \\
\hline & $\rho_{\mathrm{G} 91}$ \\
\hline & $\rho_{\mathrm{G} 91}$ \\
\hline & $\rho_{\mathrm{G} 91}$ \\
\hline & $\rho_{508}$ \\
\hline & $\rho_{508}$ \\
\hline
\end{tabular}

$Q_{400 \text { C.1dpa. } Y_{i}}:=\rho_{400 \text { C.1dpa.Y. }} \cdot \mathrm{HR}_{400 \text { C.1dpa.Y }}$ 


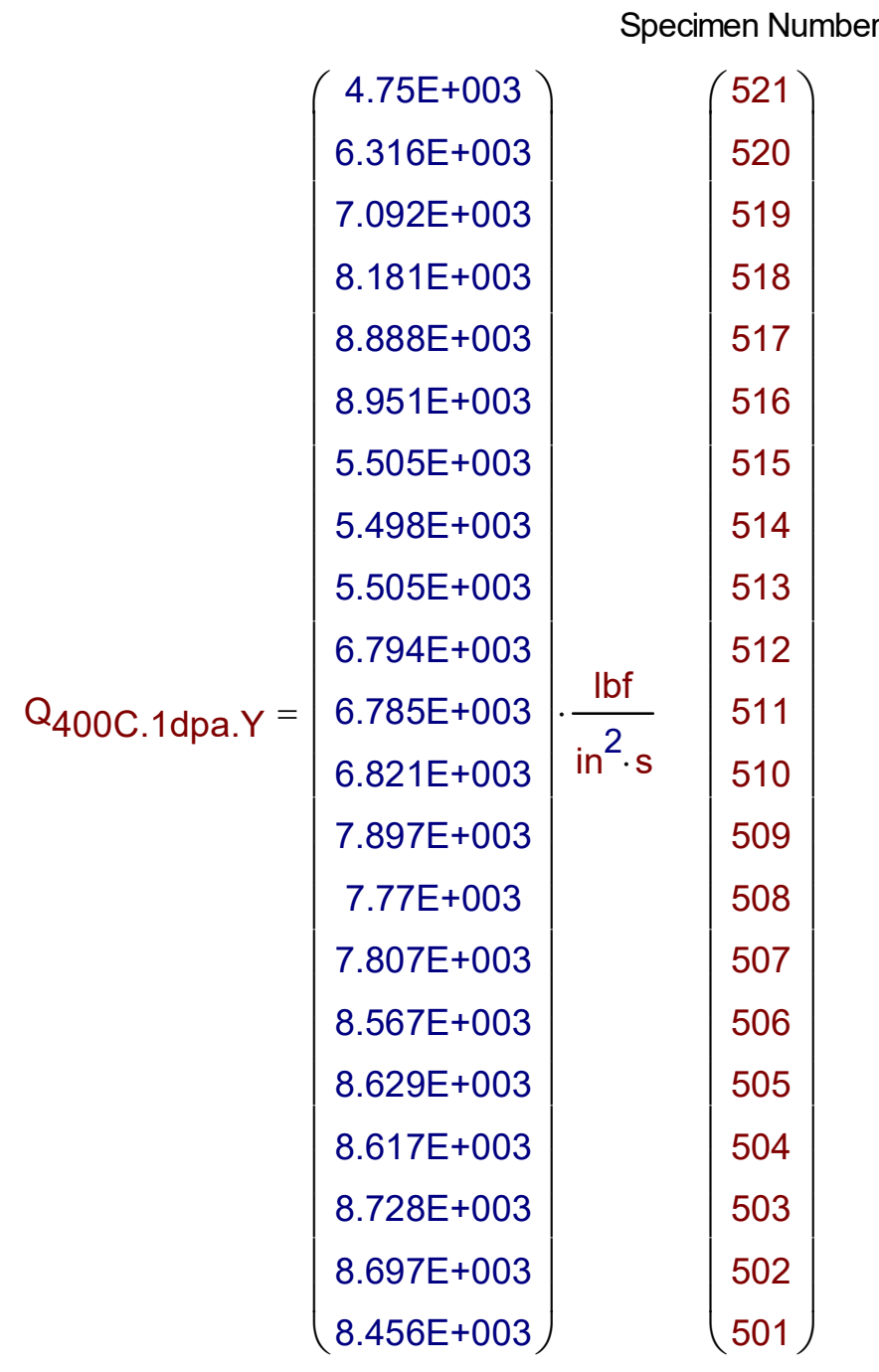




\section{B.7 Heating Rates for Capsule 400C 3 DPA (X):}

Specimen Number

\begin{tabular}{|c|c|c|c|}
\hline 5.25 & 5.83 & 5.89 & $(674)$ \\
\hline 6.23 & 6.92 & 6.99 & 673 \\
\hline 7.33 & 8.15 & 8.23 & 672 \\
\hline 7.92 & 8.8 & 8.89 & 671 \\
\hline 7.63 & 8.48 & 8.56 & 670 \\
\hline 8.66 & 9.63 & 9.72 & 669 \\
\hline 8.7 & 9.67 & 9.77 & 668 \\
\hline 7.77 & 8.64 & 8.72 & 667 \\
\hline 7.86 & 8.73 & 8.82 & 666 \\
\hline 7.84 & 8.71 & 8.8 & 665 \\
\hline 7.94 & 8.82 & 8.91 & 664 \\
\hline 8.13 & 9.04 & 9.13 & 663 \\
\hline 8.02 & 8.92 & 9 & 662 \\
\hline 7.91 & 8.8 & 8.88 & 661 \\
\hline 7.92 & 8.8 & 8.88 & 660 \\
\hline 8.1 & 9 & 9.09 & 659 \\
\hline 8.02 & 8.92 & 9 & 658 \\
\hline 7.83 & 8.71 & 8.79 & 657 \\
\hline 7.85 & 8.73 & 8.81 & 656 \\
\hline 7.72 & 8.58 & 8.66 & 655 \\
\hline 7.79 & 8.66 & 8.74 & 654 \\
\hline 7.69 & 8.55 & 8.63 & 653 \\
\hline 7.73 & 8.59 & 8.68 & 652 \\
\hline 7.7 & 8.56 & 8.64 & 651 \\
\hline 7.84 & 8.72 & 8.8 & 650 \\
\hline 8.01 & 8.9 & 8.99 & 649 \\
\hline 7.92 & 8.8 & 8.89 & 648 \\
\hline 7.86 & 8.74 & 8.82 & 647 \\
\hline 7.85 & 8.73 & 8.81 & 646 \\
\hline 7.88 & 8.76 & 8.85 & 645 \\
\hline 7.89 & 8.77 & 8.85 & 644 \\
\hline 8.06 & 8.96 & 9.04 & 643 \\
\hline 8.16 & 9.07 & 9.15 & 642 \\
\hline
\end{tabular}




\section{BSU-8242 As-Run Thermal Analysis}

\begin{tabular}{|c|c|c|c|c|c|}
\hline \multirow{36}{*}{$\mathrm{HR}_{400 \mathrm{C} .3 \mathrm{dpa} . \mathrm{X}}:=$} & 8.16 & 9.07 & 9.16 & & 641 \\
\hline & 8.23 & 9.15 & 9.24 & & 640 \\
\hline & 8.07 & 8.97 & 9.05 & & 639 \\
\hline & 8.04 & 8.94 & 9.02 & W & 638 \\
\hline & 7.86 & 8.74 & 8.82 & $\overline{\mathrm{gm}}$ & 637 \\
\hline & 7.88 & 8.76 & 8.84 & & 636 \\
\hline & 7.94 & 8.82 & 8.91 & & 635 \\
\hline & 7.86 & 8.73 & 8.82 & & 634 \\
\hline & 7.91 & 8.79 & 8.88 & & 633 \\
\hline & 7.89 & 8.78 & 8.86 & & 632 \\
\hline & 8.06 & 8.96 & 9.04 & & 631 \\
\hline & 8.16 & 9.07 & 9.16 & & 630 \\
\hline & 8.13 & 9.04 & 9.13 & & 629 \\
\hline & 8.05 & 8.94 & 9.03 & & 628 \\
\hline & 8.19 & 9.11 & 9.19 & & 627 \\
\hline & 8.16 & 9.07 & 9.15 & & 626 \\
\hline & 8.2 & 9.12 & 9.21 & & 625 \\
\hline & 8.09 & 8.99 & 9.08 & & 624 \\
\hline & 8.06 & 8.96 & 9.05 & & 623 \\
\hline & 8.16 & 9.07 & 9.16 & & 622 \\
\hline & 8.14 & 9.05 & 9.13 & & 621 \\
\hline & 8.13 & 9.04 & 9.12 & & 620 \\
\hline & 8.62 & 9.58 & 9.67 & & 619 \\
\hline & 8.67 & 9.64 & 9.73 & & 618 \\
\hline & 8.61 & 9.57 & 9.66 & & 617 \\
\hline & 8.73 & 9.71 & 9.8 & & 616 \\
\hline & 8.69 & 9.66 & 9.76 & & 615 \\
\hline & 8.74 & 9.72 & 9.81 & & 614 \\
\hline & 8.63 & 9.59 & 9.68 & & 613 \\
\hline & 8.55 & 9.51 & 9.6 & & 612 \\
\hline & 8.63 & 9.6 & 9.69 & & 611 \\
\hline & 8.53 & 9.49 & 9.58 & & 610 \\
\hline & 8.63 & 9.59 & 9.68 & & 609 \\
\hline & 8.52 & 9.47 & 9.56 & & 608 \\
\hline & 8.3 & 9.22 & 9.31 & & 607 \\
\hline & 8.32 & 9.25 & 9.33 & & 606 \\
\hline
\end{tabular}




$\left.\begin{array}{l}\left(\begin{array}{ccc}7.7 & 8.56 & 8.64 \\ 8.11 & 9.02 & 9.11 \\ 7.66 & 8.52 & 8.6 \\ 6.69 & 7.44 & 7.51 \\ 5.82 & 6.47 & 6.53\end{array}\right) \\ i:=0 . . \text { length }\left(H_{400 C .3 d p a . X}^{\langle 0\rangle}\right)-1 \\ j:=0 . .2\end{array} \mid \begin{array}{l}605 \\ 604 \\ 603 \\ 602 \\ 601\end{array}\right)$

$\left(\begin{array}{c}\rho_{690} \\ \rho_{690} \\ \rho_{625} \\ \rho_{625} \\ \rho_{\mathrm{G} 91} \\ \rho_{625} \\ \rho_{625} \\ \rho_{\mathrm{G} 91} \\ \rho_{\mathrm{G} 91} \\ \rho_{\mathrm{G} 91} \\ \rho_{\mathrm{G} 91} \\ \rho_{\mathrm{G} 91} \\ \rho_{\mathrm{G} 91} \\ \rho_{304 \mathrm{~L}} \\ \rho_{304 \mathrm{~L}} \\ \rho_{304 \mathrm{~L}} \\ \rho_{304 \mathrm{~L}} \\ \rho_{304 \mathrm{~L}} \\ \rho_{304 \mathrm{~L}} \\ \rho_{304 \mathrm{~L}} \\ \rho_{304 \mathrm{~L}} \\ \rho_{304 \mathrm{~L}} \\ \rho_{304 \mathrm{~L}}\end{array}\right)$




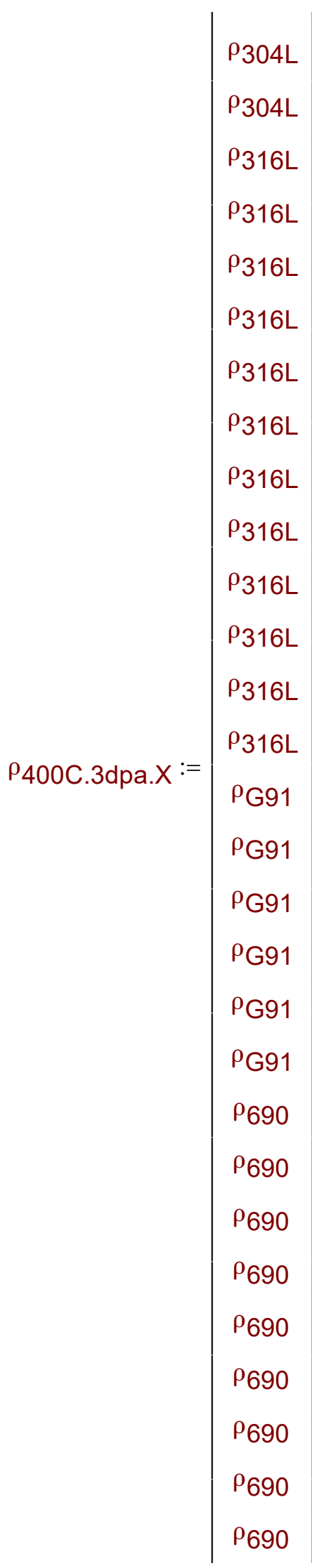




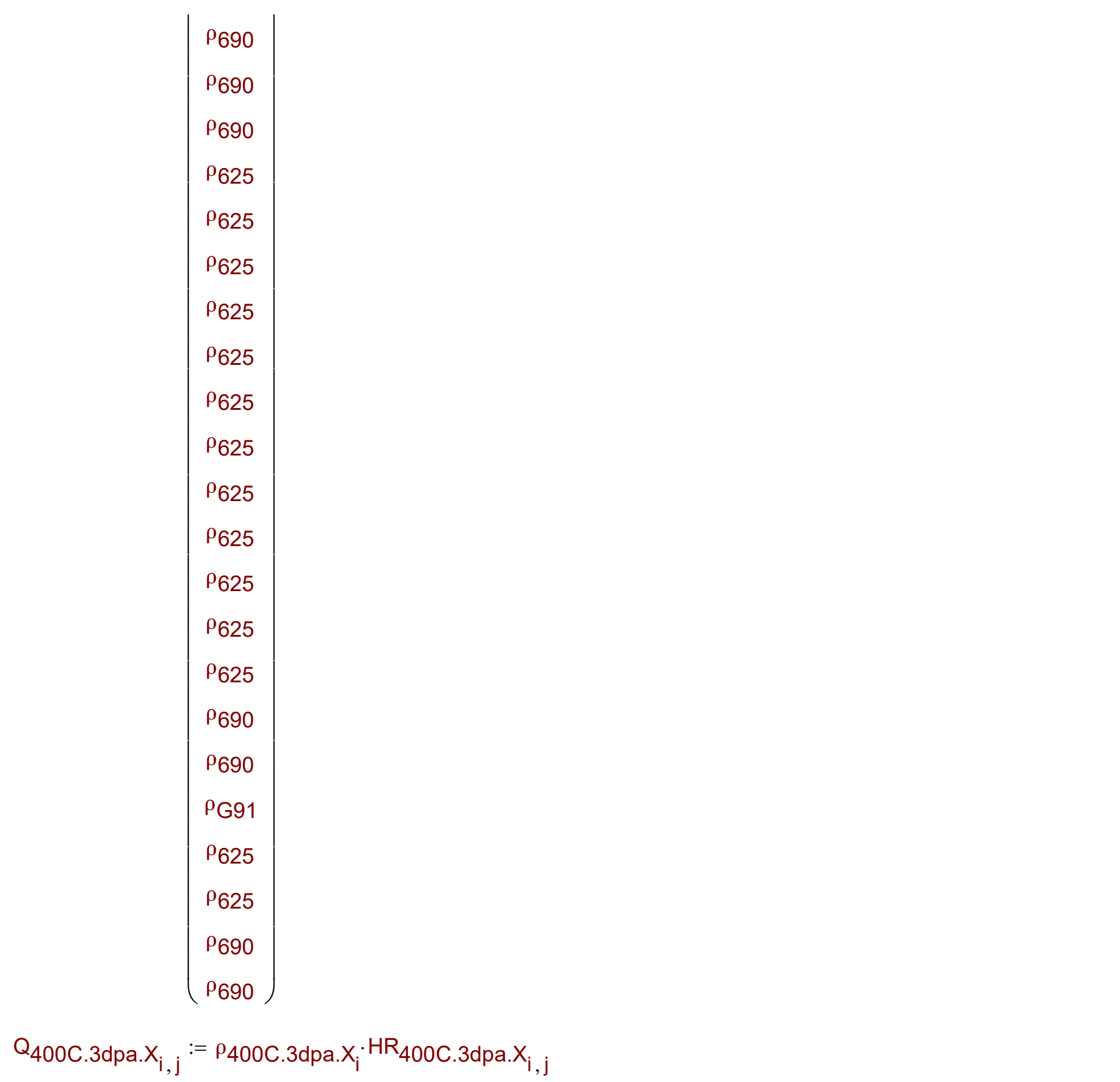


BSU-8242 As-Run Thermal Analysis

$\left(\begin{array}{ccc}6.236 E+003 & 6.925 E+003 & 6.996 E+003 \\ 7.4 E+003 & 8.22 E+003 & 8.303 E+003 \\ 8.973 E+003 & 9.977 E+003 & 1.007 E+004 \\ 9.695 E+003 & 1.077 E+004 & 1.088 E+004 \\ 8.637 E+003 & 9.6 E+003 & 9.69 E+003 \\ 1.06 E+004 & 1.179 E+004 & 1.19 E+004 \\ 1.065 E+004 & 1.184 E+004 & 1.196 E+004 \\ 8.796 E+003 & 9.781 E+003 & 9.871 E+003 \\ 8.898 E+003 & 9.883 E+003 & 9.984 E+003 \\ 8.875 E+003 & 9.86 E+003 & 9.962 E+003 \\ 8.988 E+003 & 9.984 E+003 & 1.009 E+004 \\ 9.203 E+003 & 1.023 E+004 & 1.034 E+004 \\ 9.079 E+003 & 1.01 E+004 & 1.019 E+004 \\ 9.212 E+003 & 1.025 E+004 & 1.034 E+004 \\ 9.224 E+003 & 1.025 E+004 & 1.034 E+004 \\ 9.434 E+003 & 1.048 E+004 & 1.059 E+004 \\ 9.341 E+003 & 1.039 E+004 & 1.048 E+004 \\ 9.119 E+003 & 1.014 E+004 & 1.024 E+004 \\ 9.143 E+003 & 1.017 E+004 & 1.026 E+004 \\ 8.991 E+003 & 9.993 E+003 & 1.009 E+004 \\ 9.073 E+003 & 1.009 E+004 & 1.018 E+004 \\ 8.956 E+003 & 9.958 E+003 & 1.005 E+004 \\ 9.003 E+003 & 1 E+004 & 1.011 E+004 \\ 8.968 E+003 & 9.969 E+003 & 1.006 E+004 \\ 9.131 E+003 & 1.016 E+004 & 1.025 E+004 \\ 9.282 E+003 & 1.031 E+004 & 1.042 E+004 \\ 9.178 E+003 & 1.02 E+004 & 1.03 E+004 \\ 9.109 E+003 & 1.013 E+004 & 1.022 E+004 \\ 9.097 E+003 & 1.012 E+004 & 1.021 E+004 \\ 9.132 E+003 & 1.015 E+004 & 1.026 E+004 \\ 9.143 E+003 & 1.016 E+004 & 1.026 E+004 \\ 9.34 E+003 & 1.038 E+004 & 1.048 E+004 \\ 9.456 E+003 & 1.051 E+004 & 1.06 E+004 \\ 9.456 E+003 & 1.051 E+004 & 1.062 E+004 \\ 9.537 E+003 & 1.06 E+004 & 1.071 E+004\end{array} \mid\right.$

Specimen Number

$\left.\begin{array}{l}674 \\ 673 \\ 672 \\ 671 \\ 670 \\ 669 \\ 668 \\ 667 \\ 666 \\ 665 \\ 664 \\ 663 \\ 662 \\ 661 \\ 660 \\ 659 \\ 658 \\ 657 \\ 656 \\ 655 \\ 654 \\ 653 \\ 652 \\ 651 \\ 650 \\ 649 \\ 648 \\ 647 \\ 646 \\ 645 \\ 644 \\ 643 \\ 642 \\ 641 \\ 640\end{array}\right)$


BSU-8242 As-Run Thermal Analysis

\begin{tabular}{|c|c|c|c|c|c|}
\hline \multirow{36}{*}{$Q_{400 C .3 d p a . X}=$} & $9.352 E+003$ & $1.039 E+004$ & $1.049 E+004$ & \multirow{3}{*}{$\frac{\mathrm{lbf}}{\mathrm{in}^{2} \cdot \mathrm{s}}$} & 639 \\
\hline & $9.317 E+003$ & $1.036 \mathrm{E}+004$ & $1.045 E+004$ & & 638 \\
\hline & $8.898 E+003$ & $9.894 E+003$ & $9.984 E+003$ & & 637 \\
\hline & $8.92 E+003$ & $9.916 E+003$ & $1.001 E+004$ & & 636 \\
\hline & $8.988 E+003$ & $9.984 E+003$ & $1.009 E+004$ & & 635 \\
\hline & $8.898 E+003$ & $9.883 E+003$ & $9.984 E+003$ & & 634 \\
\hline & $8.954 E+003$ & $9.95 E+003$ & $1.005 E+004$ & & 633 \\
\hline & $8.932 E+003$ & $9.939 E+003$ & $1.003 E+004$ & & 632 \\
\hline & $9.574 \mathrm{E}+003$ & $1.064 \mathrm{E}+004$ & $1.074 E+004$ & & 631 \\
\hline & $9.693 E+003$ & 1.077E+004 & $1.088 E+004$ & & 630 \\
\hline & $9.657 E+003$ & $1.074 \mathrm{E}+004$ & $1.085 E+004$ & & 629 \\
\hline & $9.562 E+003$ & $1.062 \mathrm{E}+004$ & $1.073 E+004$ & & 628 \\
\hline & $9.729 E+003$ & $1.082 E+004$ & $1.092 E+004$ & & 627 \\
\hline & $9.693 E+003$ & $1.077 E+004$ & $1.087 E+004$ & & 626 \\
\hline & $9.74 \mathrm{E}+003$ & $1.083 E+004$ & $1.094 E+004$ & & 625 \\
\hline & $9.61 \mathrm{E}+003$ & $1.068 \mathrm{E}+004$ & $1.079 E+004$ & & 624 \\
\hline & $9.574 \mathrm{E}+003$ & $1.064 \mathrm{E}+004$ & $1.075 E+004$ & & 623 \\
\hline & $9.693 E+003$ & $1.077 E+004$ & $1.088 E+004$ & & 622 \\
\hline & $9.669 \mathrm{E}+003$ & $1.075 E+004$ & $1.085 E+004$ & & 621 \\
\hline & $9.657 \mathrm{E}+003$ & $1.074 \mathrm{E}+004$ & $1.083 E+004$ & & 620 \\
\hline & $1.055 E+004$ & $1.173 E+004$ & $1.184 \mathrm{E}+004$ & & 619 \\
\hline & $1.061 \mathrm{E}+004$ & 1.18E+004 & 1.191E+004 & & 618 \\
\hline & $1.054 \mathrm{E}+004$ & $1.171 \mathrm{E}+004$ & $1.182 E+004$ & & 617 \\
\hline & $1.069 E+004$ & $1.189 E+004$ & $1.2 \mathrm{E}+004$ & & 616 \\
\hline & $1.064 \mathrm{E}+004$ & $1.182 E+004$ & 1.195E+004 & & 615 \\
\hline & 1.07E+004 & $1.19 \mathrm{E}+004$ & $1.201 E+004$ & & 614 \\
\hline & $1.056 \mathrm{E}+004$ & $1.174 \mathrm{E}+004$ & $1.185 E+004$ & & 613 \\
\hline & $1.047 E+004$ & $1.164 \mathrm{E}+004$ & $1.175 E+004$ & & 612 \\
\hline & $1.056 \mathrm{E}+004$ & $1.175 E+004$ & 1.186E+004 & & 611 \\
\hline & $1.044 E+004$ & $1.162 E+004$ & $1.173 E+004$ & & 610 \\
\hline & $1.056 \mathrm{E}+004$ & $1.174 \mathrm{E}+004$ & $1.185 E+004$ & & 609 \\
\hline & $1.043 E+004$ & $1.159 E+004$ & $1.17 E+004$ & & 608 \\
\hline & $9.859 E+003$ & $1.095 E+004$ & 1.106E+004 & & 607 \\
\hline & $9.883 E+003$ & $1.099 E+004$ & $1.108 E+004$ & & 606 \\
\hline & 8.717E+003 & $9.69 E+003$ & $9.781 E+003$ & & 605 \\
\hline & $9.928 \mathrm{E}+003$ & $1.104 E+004$ & $1.115 E+004$ & & 604 \\
\hline
\end{tabular}


BSU-8242 As-Run Thermal Analysis

$\left(\begin{array}{lll}9.377 E+003 & 1.043 E+004 & 1.053 E+004 \\ 7.947 E+003 & 8.838 E+003 & 8.921 E+003 \\ 6.913 E+003 & 7.685 E+003 & 7.757 E+003\end{array}\right) \quad\left(\begin{array}{l}603 \\ 602 \\ 601\end{array}\right)$

\section{B.8 Heating Rates for Capsule 400C 3 DPA (Y):}

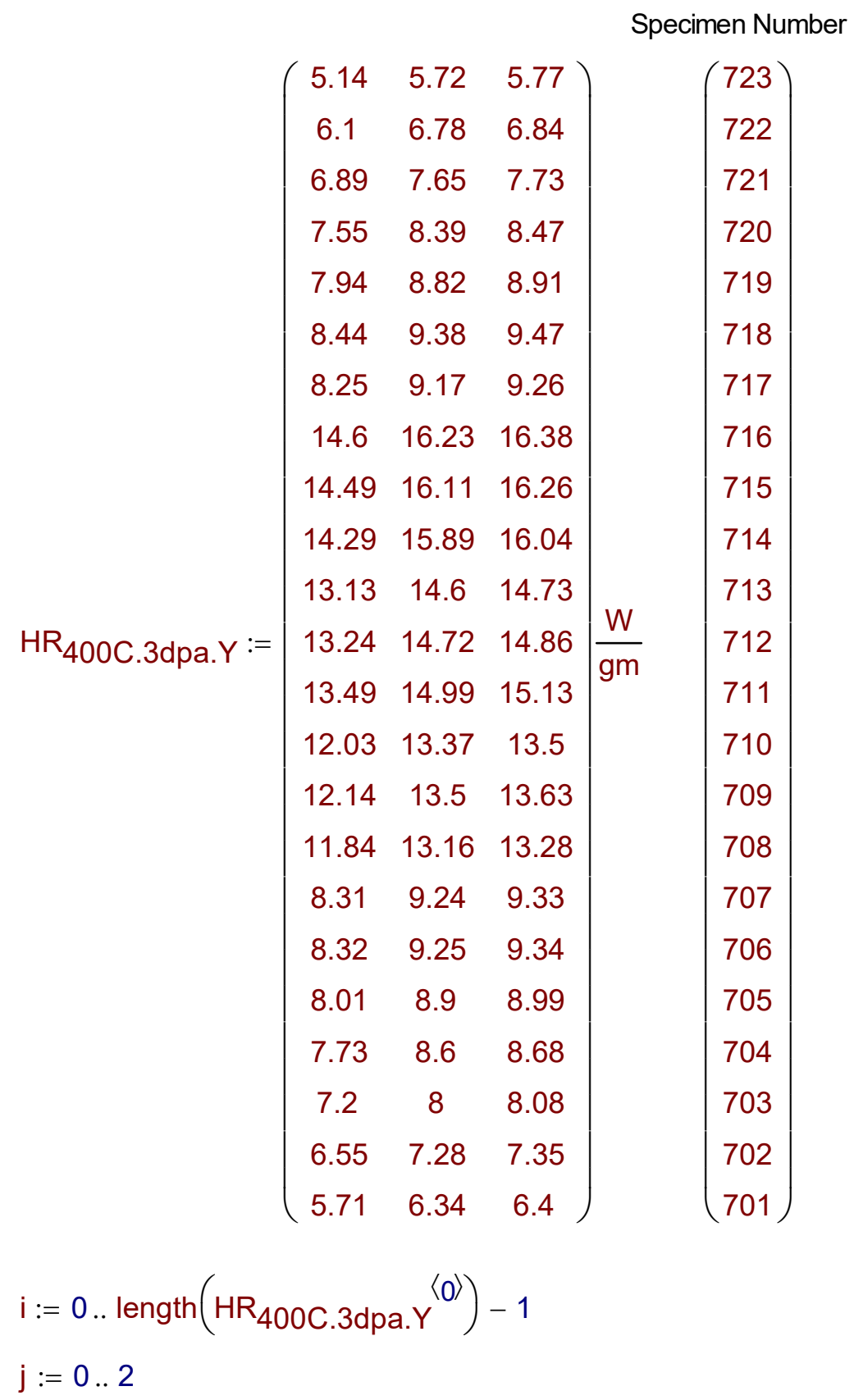




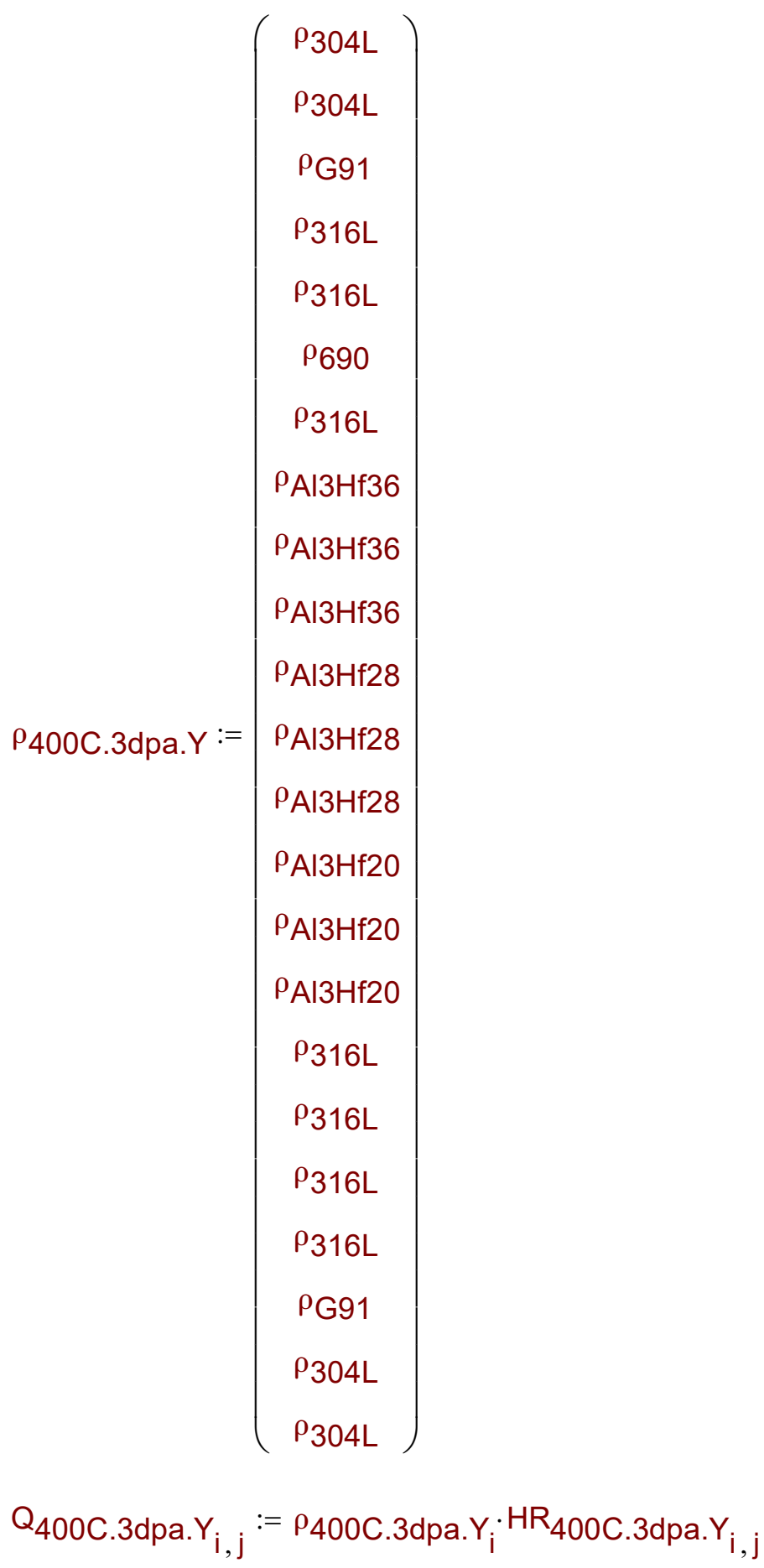




Q $_{400 C .3 d p a . Y}=\left(\begin{array}{ccc}5.986 E+003 & 6.662 E+003 & 6.72 E+003 \\ 7.104 E+003 & 7.896 E+003 & 7.966 E+003 \\ 7.8 E+003 & 8.66 E+003 & 8.751 E+003 \\ 8.749 E+003 & 9.723 E+003 & 9.815 E+003 \\ 9.201 E+003 & 1.022 E+004 & 1.033 E+004 \\ 1.003 E+004 & 1.114 E+004 & 1.125 E+004 \\ 9.561 E+003 & 1.063 E+004 & 1.073 E+004 \\ 8.364 E+003 & 9.298 E+003 & 9.384 E+003 \\ 8.301 E+003 & 9.229 E+003 & 9.315 E+003 \\ 8.187 E+003 & 9.103 E+003 & 9.189 E+003 \\ 7.122 E+003 & 7.92 E+003 & 7.99 E+003 \\ 7.182 E+003 & 7.985 E+003 & 8.061 E+003 \\ 7.318 E+003 & 8.131 E+003 & 8.207 E+003 \\ 5.981 E+003 & 6.647 E+003 & 6.712 E+003 \\ 6.036 E+003 & 6.712 E+003 & 6.777 E+003 \\ 5.887 E+003 & 6.543 E+003 & 6.603 E+003 \\ 9.63 E+003 & 1.071 E+004 & 1.081 E+004 \\ 9.642 E+003 & 1.072 E+004 & 1.082 E+004 \\ 9.282 E+003 & 1.031 E+004 & 1.042 E+004 \\ 8.958 E+003 & 9.966 E+003 & 1.006 E+004 \\ 8.151 E+003 & 9.056 E+003 & 9.147 E+003 \\ 7.628 E+003 & 8.479 E+003 & 8.56 E+003 \\ 6.65 E+003 & 7.384 E+003 & 7.454 E+003\end{array}\right) \cdot \quad$ Ibf $\quad\left(\begin{array}{l}723 \\ 722 \\ 721 \\ 720 \\ 719 \\ 718 \\ 717 \\ 716 \\ 715 \\ 714 \\ 713 \\ 712 \\ 711 \\ 710 \\ 709 \\ 708 \\ 707 \\ 706 \\ 705 \\ 704 \\ 703 \\ 702 \\ 701\end{array}\right)$


Appendix C

\section{Computer Code Validation}


ABQ EXE: abq6142

COMPUTER: falcon 1

OS: Linux

OS TYPE: 3.0.101-108.77-defau1t

t1

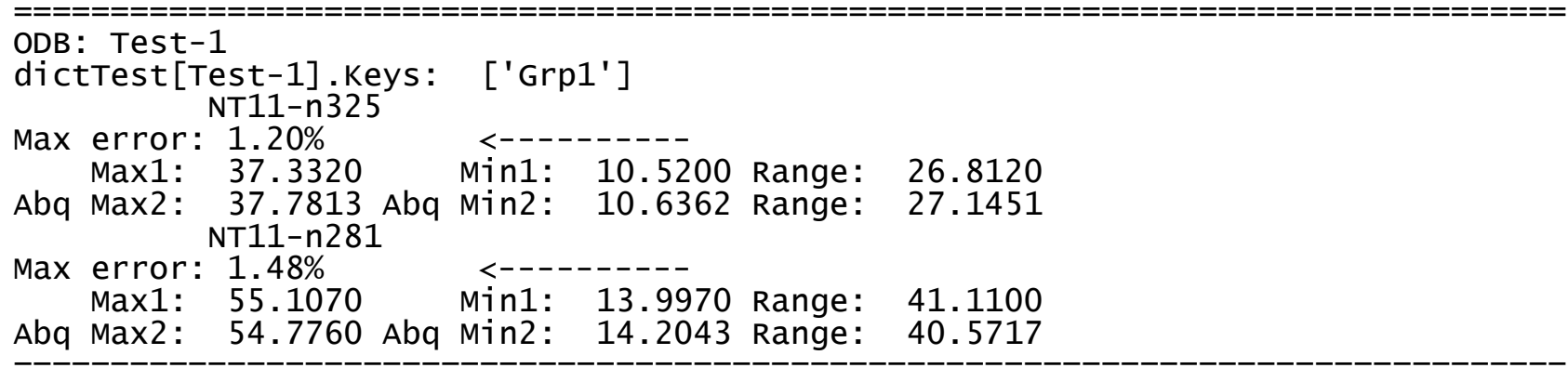

t2

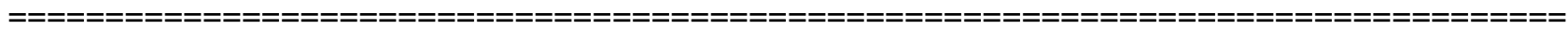
ODB: Test-2

dictTest[Test-2].Keys: ['Grp2', 'Grp1'] NT15-n61

Max error: $1.34 \%$ Max1: 37.3320 Min1: 10.5200 Range: 26.8120

Abq Max2: 37.7366 Abq Min2: 10.6609 Range: 27.0756

NT11-n61

Max1: 55.1070 Min1: 13.9970 Range: 41.1100

Abq Max2: 54.7444 Abq Min2: 14.2131 Range: 40.5313

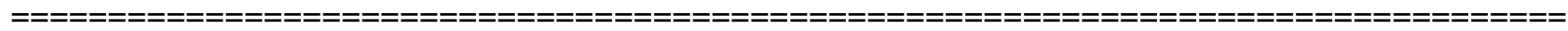

t3

ODB: Test -3

dictTest [Test-3].Keys: ['Grp1']

NT11-n130

Max error: $1.65 \%$

Max1: 44.5920 Min1: 12.5210 Range: 32.0710

Abq Max2: 44.7825 Abq Min2: 12.7270 Range: 32.0555 NT11-n59

Max error: $1.85 \%$

Max1: 55.3390 Min1: 14.7770 Range: 40.5620

Abq Max2: 55.0396 Abq Min2: 15.0511 Range: 39.9885

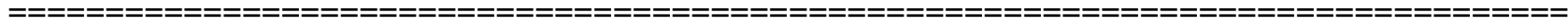

t4

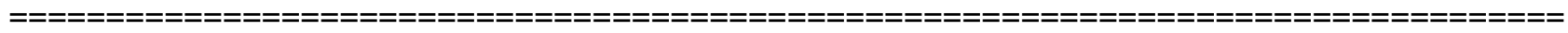
ODB: Test-4

dictTest[Test-4].Keys: ['Grp1'] NT11-n281

Error: $0.00 \%$

Ans: $\quad 13.7600$

Error: $0.00 \%$ : $11-n 303$

Ans: 11.3200 Abq: 11.3200

$\begin{array}{lcc}\text { Error: } & 0.00 \% \text { H } & \\ \text { Ans: } & 4.0000 & \text { Abq: }\end{array}$ 


\begin{tabular}{|cc|}
\hline $\begin{array}{c}\text { TEM-10200-1, Rev. } 11 \\
11 / 20 / 2019\end{array}$ & ENGINEERING CALCULATIONS AND ANALYSIS \\
BSev. 0 \\
Page C3 of C4 4
\end{tabular}

$\begin{array}{lcc}\text { Error: } & 0.00 \% \quad 8.2700 \quad \text { Abq: } & 8.2700 \\ \text { Ans: } & \text { NT11 }-\mathrm{n} 292 & \end{array}$

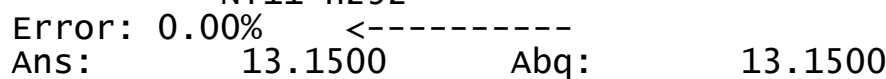

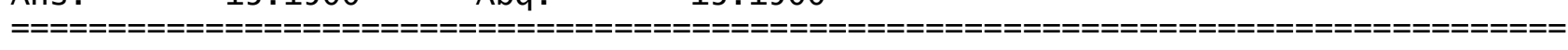

t5

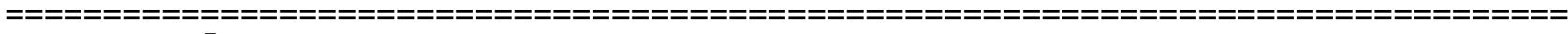
ODB: Test-5

dictTest[Test-5].Keys: ['Grp3', 'Grp2', 'Grp1', 'Grp5', 'Grp4']

$\begin{array}{lcc}\text { Error: } & 0.00 \% & \\ \text { Ans: } & 11.3200 & \text { Abq: }\end{array}$ NT13-n62

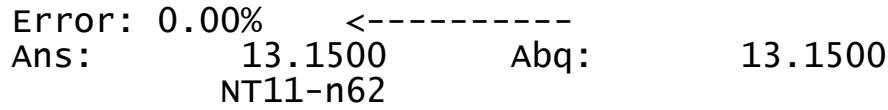

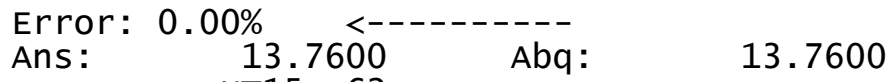

$\begin{array}{lrr}13.7600 & \text { Abq: } & 13.7600\end{array}$

Ans: $\quad 4.0000 \quad \mathrm{Abq}$ : 4.0000

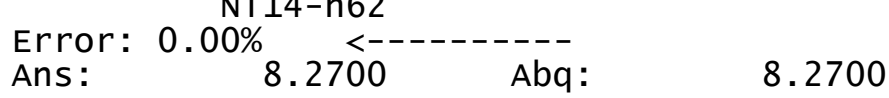

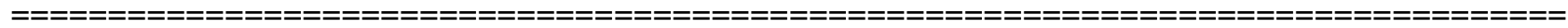

t6

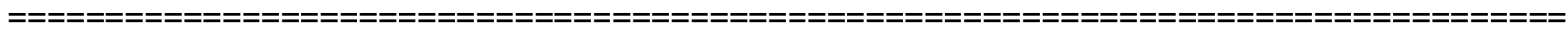

ODB: Test-6

dictTest [Test-6]. Keys: ['Grp1'] NT11-n533

Max error: $0.39 \%$ Max1: 80.7640 Min1: 61.8970 Range: 18.8670

Abq Max2: 80.4914 Abq Min2: 61.7364 Range: 18.7551 NT11-n803

Max error: $0.38 \%$

Max1: $94.5930 \quad$ Min1: 71.5310 Range: 23.0620

Abq Max2: 94.3007 Abq Min2: 71.2781 Range: 23.0226

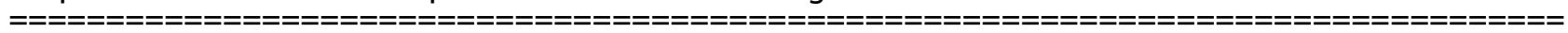

t7

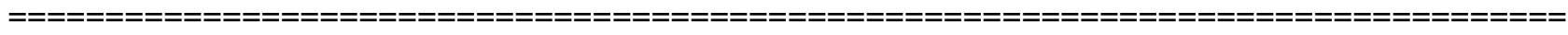
ODB: Test-7

dictTest [Test-7]. Keys: ['Grp1']

Error: $0.19 \%$ HFL-e 56

Ans: $\quad-0.1700 \quad$ Abq: $\quad-0.1697$

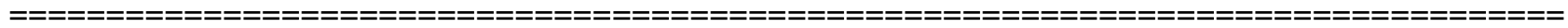

t8

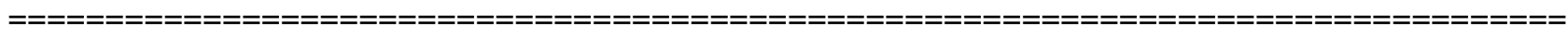

ODB: Test-8

dictTest [Test-8]. Keys: ['Grp1'] HFL-e1121

Error: $1.74 \%$

Ans: $\quad 0.1710 \quad \mathrm{Abq}: \quad 0.1740$

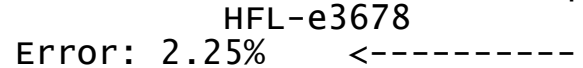

Ans: $\quad-0.1620 \quad$ Abq: $\quad-0.1656$

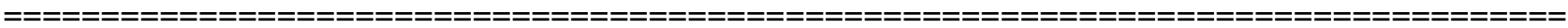


t9

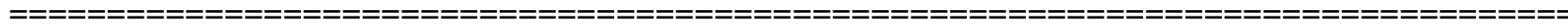
ODB: Test-9

dictTest [Test-9]. Keys: ['Grp1']

Error: $0.01 \%$ NT11-n13

Ans: $\begin{array}{rr}50.0010 \\ \text { NT11-n17 }\end{array} \quad$ Abq: $\quad 50.0036$

Error: $0.00 \%$

Ans: $\quad 55.5500 \quad$ Abq: $\quad 55.5500$

Ans: $\quad 0.20 \%$ $51.6040 \quad$ Abq: $\quad 51.7074$

NT11-n38

Ans: $\quad 50.0890 \quad$ Abq: $\quad 50.1148$

Error: $0.11 \%$

Ans: $\quad 50.7010 \quad$ Abq: $\quad 50.7550$

Error: $0.01 \%$

Ans: $\begin{array}{ccc}50.0110 & \text { Abq: } & 50.0176\end{array}$

Error: $0.10 \%$

Ans: $\quad 50.3060 \quad$ Abq: $\quad 50.3555$

NT11-n324

Ans: $\quad 52.4260$ Abq: 52.5321

Error: $0.08 \% 11-\mathrm{n} 4$

Ans: $\quad 51.0600$ Abq: 51.1006

Error: $0.16 \%$ :

Ans: $\quad 53.6690 \quad$ Abq: $\quad 53.7552$

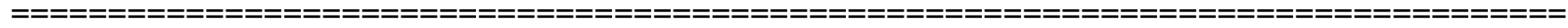

t10

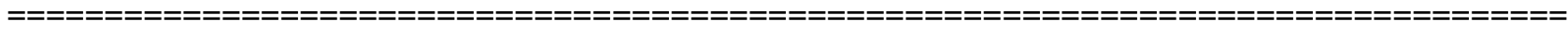

ODB: Test-10

dictTest [Test-10]. Keys: ['Grp1']

NT11-n325

Error: $0.15 \%$

Ans: $\quad 215.7130 \quad$ Abq: 216.0345

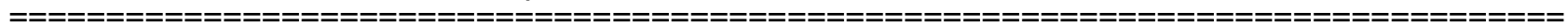

\section{t11}

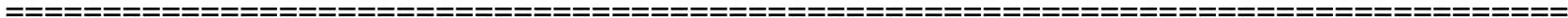
ODB: Test-11

dictTest [Test-11].Keys: ['Grp1']

Error: $0.02 \%$ <--.--.-

Ans: $\quad-5.5000 \quad$ Abq: $\quad-5.4989$

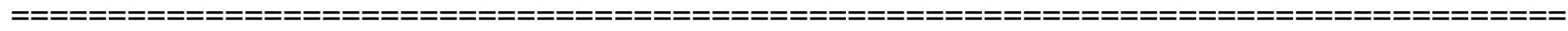

$\mathrm{t} 12$

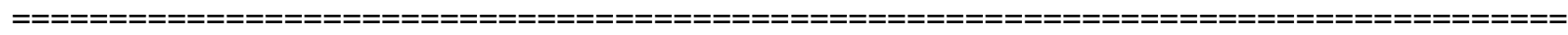
ODB: Test-12

dictTest [Test-12].Keys: ['Grp1']

NT11-n336

Error: $0.00 \%$

Ans: $\quad 406.6667$

Abq:

406.6667

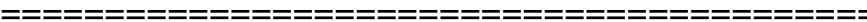

